\title{
Une formule de Riemann-Roch équivariante pour les courbes
}

\author{
Niels Borne
}

Version du 31 janvier 2000 


\section{Remerciements}

Avant tout, je tiens à remercier mon directeur de thèse Boas Erez. Il a su non seulement me soumettre une problématique riche de perspectives et d'enseignements mais aussi insuffler sans cesse de nouvelles idées dans mon sujet. De cette manière, il m'a fait profiter de sa vision de la théorie géométrique des modules galoisiens, et de la géomètrie arithmétique en général.

Je suis très reconnaissant envers Bernhard Köck et Angelo Vistoli qui ont accepté d'être les rapporteurs de cette thèse, et également de faire partie de mon jury. A cette occasion, et aussi lors de multiples conversations, j'ai pu apprécier l'attention qu'ils portent à mon travail. Je les en remercie vivement.

Je remercie Philippe Cassou-Noguès et Joseph Tapia pour leur écoute, et aussi pour avoir accepté d'être membres de mon jury.

Cette thèse a été conçue et rédigée dans le cadre très convivial du Laboratoire de Mathématiques Pures de l'Université Bordeaux 1. Je tiens à exprimer ma reconnaissance aux membres du groupe de géomètrie arithmétique, et en particulier à Quing Liu et à Michel Matignon. Je tiens également à remercier Mauricette Jaubert, de la reprographie, Joëlle Pargade, de l'école doctorale, et Francine Delmer, du service culturel, pour leur disponibilité et leur bonne humeur.

En cet instant, j'ai évidemment une pensée pour mon professeur de collège, Mme Deutsch, et pour mes professeurs de classes préparatoires, Mrs Beck et Simon, qui ont su me faire partager leur passion des mathématiques avec enthousiasme. Je leur en serai toujours reconnaissant.

Il me reste à remercier ceux qui m'ont entouré pendant ces trois années. Pour n'en citer que quelques-uns (que les autres me pardonnent) merci donc à Stéphane, Carine, Yannick, Bidou, Joëlle, Gilles, Hugo, Hakim, Manu, Eric, Frédéric, Philippe et Irène pour les nombreuses conversations amicales que nous avons eues, et pour bien d'autres choses encore. 


\section{Table des matières}

1 Structures galoisiennes et ramification 11

1.1 Introduction . . . . . . . . . . . . . . . . . . 11

1.2 Deux applications de l'étude des structures galoisiennes . . . . . . 12

1.2.1 Conditions d'existence d'actions libres . . . . . . . . 12

1.2.2 Singularités des espaces des modules grossier des courbes . 12

1.3 Résultats du type Noether . . . . . . . . . . . . . . . . . . 13

1.4 Résultats du type Riemann-Roch . . . . . . . . . . . . . . . . 14

1.4.1 Formule de Chevalley-Weil . . . . . . . . . . . . . . 15

1.4 .2 Formule de Weil . . . . . . . . . . . . . . . . . . . . 17

1.4.3 Formule de Lefschetz . . . . . . . . . . . . . . . . . 17

1.4.4 Formules déduites du formalisme de Riemann-Roch . . . . 18

$2 G$-faisceaux 21

2.1 Généralités . . . . . . . . . . . . . . . . . . . . 21

2.1 .1 Conventions . . . . . . . . . . . . . . . . . . . . 21

2.1 .2 Définition . . . . . . . . . . . . . . . . . . . 21

2.1 .3 Exemples . . . . . . . . . . . . . . . . . . . . . . 22

2.2 Catégorie des $G$-faisceaux abéliens . . . . . . . . . . . . 24

2.3 Action sur les groupes de cohomologie . . . . . . . . . . 25

2.4 Formule de projection $\ldots \ldots \ldots \ldots \ldots$

2.5 Espace quotient . . . . . . . . . . . . . . . . 26

2.5 .1 Existence . . . . . . . . . . . . . . . . . 26

2.5 .2 Foncteur $\pi^{*} \ldots \ldots \ldots \ldots \ldots \ldots \ldots$

2.5 .3 Foncteur $\pi_{*}^{G} \ldots \ldots \ldots \ldots \ldots \ldots$

2.5 .4 Propriétés topologiques . . . . . . . . . . . . . . . . . 28

2.5.5 Propriétés schématiques . . . . . . . . . . . . . . 30

$2.6 \quad K$-théorie équivariante $\ldots \ldots \ldots \ldots \ldots \ldots$

2.6 .1 Introduction . . . . . . . . . . . . . . . . . . . 33

2.6 .2 Définition . . . . . . . . . . . . . . . . . 34

2.6 .3 Propriétés fonctorielles . . . . . . . . . . . . . . . 35

2.6.4 Caractéristique d'Euler-Poincaré équivariante . . . . . . 37

2.6 .5 Induction . . . . . . . . . . . . . . . . . . 38

2.6.6 Suite exacte de localisation . . . . . . . . . . . . . . 39 
2.6.7 Groupe de Grothendieck d'un schéma muni d'une action libre 40

2.6.8 Groupe de Grothendieck d'un $G$-schéma admissible et groupe de Grothendieck du quotient . . . . . . . . . . . . . . 41

2.6.9 Groupe de Grothendieck d'un $k$-schéma muni d'une action triviale .................... . . 42

2.7 Groupe de Picard équivariant . . . . . . . . . . . . . . . . . . . . . . . . 43

2.7.1 Introduction . . . . . . . . . . . . . . . . . . . 43

2.7.2 Cohomologie équivariante . . . . . . . . . . . . . . . . . . . . . 44

2.7.3 Preuve du théorème 2.34 . . . . . . . . . . . . . . 45

2.7.4 Application à l'étude de $\operatorname{Pic}_{G}(X) \ldots \ldots$. . . . . . . . . 48

3 Formule de Riemann-Roch équivariante 53

3.1 Introduction . . . . . . . . . . . . . . . . . 53

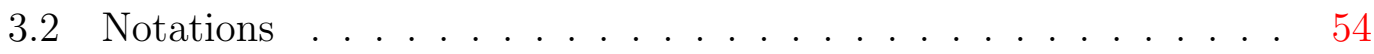

3.2.1 Définitions . . . . . . . . . . . . . . 54

3.2.2 Ramification de l'action ............. . . . . 55

3.3 Caractères de ramification . . . . . . . . . . . . . . . . . . 55

3.3.1 Définition ..................... 55

3.3.2 Cas d'une action réductive d'un groupe cyclique à ramification totale . . . . . . . . . . . 56

3.4 Degré équivariant . . . . . . . . . . . . . . . . . 57

3.4.1 Degré équivariant d'un diviseur invariant . . . . . . . . . . . . 57

3.4.2 Degré équivariant d'un $G$-faisceau inversible . . . . . . . . 57

3.5 Formule de Riemann-Roch équivariante . . . . . . . . . . . . . . 59

3.5.1 Enoncé . . . . . . . . . . . . . . . . 59

3.5.2 Démonstration . . . . . . . . . . . . . 59

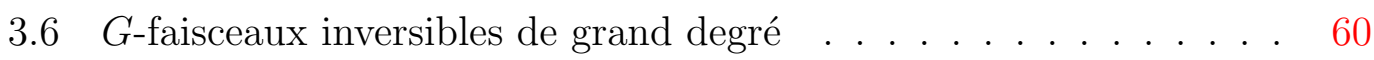

3.6.1 Projectivité de l'espace des sections globales d'un $G$-faisceau inversible de grand degré . . . . . . . . . . . . . . . . . . . 61

3.6.2 Module de ramification . . . . . . . . . . . . . . 62

3.6.3 Formule de Hurwitz équivariante . . . . . . . . . . . . . 62

3.6.4 Démonstration . . . . . . . . . . . . . . 63

3.6.5 Structure de représentation de l'espace des sections globales d'un $G$-faisceau inversible de grand degré . . . . . . . . . . 67

3.7 Sections globales du faisceau des différentielles . . . . . . . . . . . . 68

3.7.1 Enoncé du problème . . . . . . . . . . . . . . . 68

3.7.2 Degré équivariant du faisceau des différentielles et caractère d'Artin . . . . . . . . . . . . . . . . . . . . 68

3.7.3 Structure de l'espace des sections globales du faisceau des différentielles dans le cas modéré . . . . . . . . . . 70

3.7.4 Dualité équivariante et formule à la Weil . . . . . . . . . . 71

3.8 Conclusion . . . . . . . . . . . . . . . . . . . . . . 72 
4 Cycles équivariants $\quad 73$

4.1 Introduction . . . . . . . . . . . . . . . . . . . 73

4.2 Diviseurs équivariants . . . . . . . . . . . . . . . . . . . . . . . . . . . . . . . . 75

4.2.1 Définition et degré . . . . . . . . . . . . . . 75

4.2.2 Faisceau de torsion associé à un diviseur équivariant . . . . 75

4.2.3 Base en colimaçon . . . . . . . . . . . . 76

4.3 Equivalence $Y$-rationelle . . . . . . . . . . . . . . . . . . . . . . 77

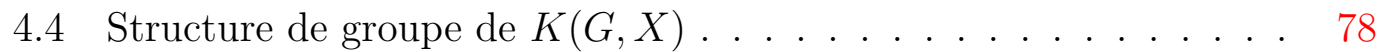

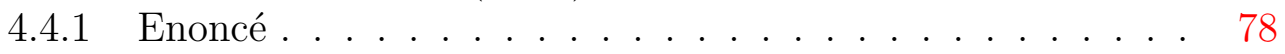

4.4.2 Démonstration . . . . . . . . . . . . . . . 79

4.5 Déterminant équivariant . . . . . . . . . . . . . . . . . . 80

4.6 Théorème de Riemann-Roch équivariant . . . . . . . . . . . . . . 81

A Algèbre homologique dans les catégories de $G$-faisceaux $\quad 83$ 


\section{Introduction}

Les courbes projectives munies d'une action d'un groupe fini sont parfois considérées comme exceptionelles ; en effet, la plupart des courbes ont un groupe d'automorphismes trivial. Cependant, et de manière quelque peu paradoxale, il est utile de considérer que les courbes avec action ont un comportement analogue à celui des courbes sans action. Pour cela, il faut se permettre de faire varier l'action du groupe considéré, pour choisir éventuellement une action triviale. Un point de vue équivalent plus agréable est de s'autoriser à changer le groupe agissant, ce qui permet par exemple de ne considérer que des actions fidèles.

Par la suite, on appelera théorie équivariante la théorie des courbes munies d'une action d'un groupe fini (éventuellement trivial), et théorie classique la théorie des courbes usuelles. D'après ce qui vient d'être dit, un énoncé en théorie équivariante fournit un énoncé en théorie classique. Il est donc légitime de se poser la question : quels sont les énoncés de la théorie classique susceptibles d'être généralisés au cas équivariant?

De ce point de vue, les énoncés issus de la théorie de l'intersection semblent prometteurs. En effet, les formules entre nombres entiers issus de cette théorie découlent souvent d'isomorphismes entre espaces vectoriels sur le corps de base de la courbe considérée. Si ces isomorphismes sont naturels, ils commuteront avec l'action et fourniront ainsi des isomorphismes entre les représentations associées, et des égalités entre les caractères correspondants. Dans une théorie équivariante des courbes, il est donc logique de remplacer les nombres entiers par des caractères.

Dans le travail qui suit, on montre comment il est possible de mettre en œuvre ces considérations élémentaires pour déduire une formule de RiemannRoch équivariante pour les courbes.

Le premier chapitre présente des résultats qui ont, d'une manière ou d'une autre, inspiré et influencé la présente thèse. Il est frappant de constater l'écart entre le théorème de Chevalley et Weil (et ses dérivés modernes) et les théorèmes issus de la technologie de la $K$-théorie équivariante. C'est ce fossé entre diverses réponses au problème de Riemann équivariant qu'on espère combler par la suite.

Le deuxième chapitre est constitué essentiellement de rappels concernant la théorie des $G$-faisceaux. Il est possible, et même préférable, de considérer des $G$ variétés de dimension arbitraire. La notion de $G$-faisceau est introduite comme 
généralisation de la notion de faisceau pour pouvoir formuler le problème de Riemann équivariant de manière précise, à savoir : quel est le caractère associé à l'espace des sections globales d'un G-faisceau donné ? Comme dans le problème de Riemann classique, on est amené à reformuler ce problème en termes de caractéristiques d'Euler. Le reste du chapitre est consacré à l'introduction de deux invariants algébriques fondamentaux attachés à chaque courbe avec action. Le premier, et certainement le plus difficile à comprendre, est le groupe de Grothendieck équivariant, dont la définition calque celle du groupe de Grothendieck classique. La raison d'être de ce groupe est, comme dans le cas classique, la suivante : les caractéristiques d'Euler équivariantes étant additives sur les suites exactes de $G$-faisceaux, on peut aborder le problème de Riemann équivariant modulo ces suites exactes. On se contente, dans un premier temps, de donner quelques propriétés classiques des groupes de Grothendieck équivariants, le problème de leur structure étant repoussé au quatrième chapitre. Le deuxième invariant algébrique associé à une courbe avec action est son groupe de Picard équivariant, qui est plus simple à comprendre. Le théorème 2.34 explicite la structure de ce groupe suivant trois points de vue : celui des $G$-faisceaux inversibles, celui des diviseurs $G$-invariants, celui des $G$-fibrés en droite.

Dans les deux derniers chapitres, on se concentre sur le cas des courbes projectives munies une action d'un groupe fini. Le troisième chapitre est consacré au problème de Riemann équivariant pour les $G$-faisceaux inversibles. On met à profit l'étude du groupe de Picard du chapitre précédent pour définir le degré équivariant d'un $G$-faisceau inversible, ce qui permet d'énoncer une formule de Riemann-Roch équivariante (théorème 3.11) pour ces faisceaux. Par la suite, on expose diverses applications de cette formule. On montre tout d'abord qu'elle fournit dans le cas d'une action modérée une réponse satisfaisante au problème de Riemann pour les $G$-faisceaux inversibles de grand degré. Enfin, on aborde le problème délicat de la structure de représentation de l'espace des sections globales des différentielles holomorphes sur la courbe. Le théorème 3.28 donne une relation concernant le caractère de Brauer de cette représentation, sans pour autant la déterminer à isomorphisme près, ce qui semble pour l'instant hors de portée dans le cas sauvage.

Dans le dernier chapitre, on examine le problème de Riemann équivariant pour les $G$-faisceaux localement libres de rang quelconque. Ceci pose le problème épineux de la définition d'une version équivariante du déterminant. Une brève étude du groupe de Grothendieck équivariant suffit à se convaincre qu'un déterminant équivariant à valeurs dans le groupe de Picard équivariant est inadéquat. En guise de substitut, on est amené à introduire un groupe de cycles à coefficients équivariants qui permet d'énoncer le théorème 4.11 qui explicite complètement la structure du groupe de Grothendieck équivariant de la courbe considérée. 


\section{Chapitre 1}

\section{Structures galoisiennes et ramification}

\subsection{Introduction}

Le but de cette première partie est d'énoncer un problème "générique" et d'examiner les réponses qu'on a pu lui donner dans des contextes particuliers.

Pour ce faire, on considère une variété projective sur un corps de base algébriquement clos et de caractéristique quelconque. On suppose de plus que cette variété est munie d'une action fidèle d'un groupe fini $G$. Une manière agréable de linéariser l'action est de considérer l'action induite sur certains groupes de cohomologie associés à la variété. On envisagera soit des groupes de cohomologie $l$-adique, soit des groupes de cohomologie de Cěch associés à des $G$-faisceaux cohérents. Les $G$-faisceaux sont par définition des faisceaux admettant une $G$ linéarisation, i.e. de manière imagée une action du groupe considéré compatible avec l'action donnée de ce groupe sur la variété fixée. Une définition précise de cette notion sera donnée au deuxième chapitre ; on pourra, dans un premier temps, prendre l'exemple des faisceaux de différentielles sur la variété. Dans ces deux cas, ces groupes de cohomologie fournissent des représentations du groupe considéré, soit sur la clôture algébrique du corps des nombres l-adiques, soit sur le corps de base de la variété.

Ici la théorie de la représentation des groupes finis intervient pour aider à préciser la structure de ces représentations d'origine géométrique. Toute représentation se décompose en somme directe finie de sous-représentations indécomposables. Les groupes de cohomologie considérés ci-dessus nous fournissent des invariants numériques, à savoir les coefficients de cette décomposition, qui sont également d'origine géométrique, et que l'on cherche à interpréter.

Les exemples donnés dans les paragraphes suivants illustrent l'idée que la structure de représentation de ces groupes de cohomologie est liée à la géométrie du lieu des points à stabilisateurs non triviaux de la variété, ou de manière 
équivalente, à la géométrie du lieu de ramification du morphisme naturel de la variété de départ vers son quotient sous l'action du groupe. Un énoncé un peu plus précis (problème 1.2) sera donné dans le paragraphe 1.3. On donne auparavant quelques applications de ce type de résultat.

\subsection{Deux applications de l'étude des structures galoisiennes}

\subsubsection{Conditions d'existence d'actions libres}

Une première application est de donner des obstructions à l'existence d'actions libres sur des variétés. Par exemple Browder et Katz ont souligné le fait suivant: si le genre arithmétique de la variété projective $X$ vaut 1 , alors aucun groupe fini ne peut agir librement sur $X$ ([1], Corollary 1.4). Ceci est une simple conséquence de la proposition 1.1.

D'un point de vue dual, on peut étudier l'existence de revêtements galoisiens étales de groupe imposé d'une variété fixée. Autrement dit, on cherche à déterminer les quotients finis du groupe fondamental de la variété en question. Pour les courbes en caractéristique nulle, ce problème a été résolu par Grothendieck. Par contre, en caractéristique strictement positive, on n'a pas de tel critère d'existence. Cependant, l'étude des structures galoisiennes a permis à Nakajima d'énoncer le théorème suivant ([24]).

Théorème (Nakajima 1984)

Soient $Y$ une courbe projective et lisse sur un corps algébriquement clos $k, g$ le genre de $Y, G$ une groupe fini et I l'idéal d'augmentation de $k[G]$. Si $Y$ admet un revêtement étale connexe galoisien de groupe $G$ alors il existe un $k[G]$-morphisme surjectif $k[G]^{\oplus g} \rightarrow I$.

La structure galoisienne du module des différentielles sur le revêtement est en fait un résultat sous-jacent puisque ce module n'est autre que le noyau du morphisme considéré dans le théorème.

\subsubsection{Singularités des espaces des modules grossier des courbes}

Lønsted a donné une démonstration utilisant les structures galoisiennes du théorème de Rauch-Popp-Oort ([19]). Rappelons ce résultat. 


\section{Théorème (Rauch-Popp-Oort)}

Soit $M_{g}$ l'espace des modules grossier des courbes de genre $g$, et $P$ le point de $M_{g}$ correspondant à une courbe $X$.

(i) Supposons $g \geq 4$. Alors $P$ est un point singulier de $M_{g}$ si et seulement si le groupe d'automorphismes de $X$ est non trivial.

(ii) Supposons $g=3$. Alors si $X$ n'est pas hyperelliptique, $P$ est un point singulier de $M_{g}$ si et seulement si le groupe d'automorphismes de $X$ est non trivial ; si $X$ est hyperelliptique, $P$ est un point singulier de $M_{g}$ si et seulement si le groupe d'automorphismes de $X$ n'est pas d'ordre 2.

C'est cette fois-ci la structure galoisienne du fibré tangent à $X$ qui constitue l'un des point-clefs de la démonstration (voir [19] pour plus de détails).

On va à présent rappeler quelques résultats classiques en théorie des modules galoisiens. On les subdivise en deux types : les résultats du type Noether (de type qualitatif) et les résultats du type Riemann-Roch (de type explicite).

\subsection{Résultats du type Noether}

On va donner des analogues géométriques du théorème arithmétique suivant.

\section{Théorème (Emy Noether 1932)}

Soit $L / K$ une extension galoisienne de corps de nombres de groupe de Galois $G$. On a équivalence entre les deux propriétés :

(i) $L / K$ est modérement ramifiée

(ii) $\mathcal{O}_{L}$ est localement libre comme $\mathcal{O}_{K} G$-module

On commence par une proposition bien connue.

\section{Proposition 1.1}

Soit $X$ une variété projective sur un corps algébriquement clos $k, G$ un groupe fini agissant librement sur $X, \mathcal{F}$ un $G$-faisceau cohérent. La caractéristique d'Euler équivariante $\chi(G, \mathcal{F})=\sum_{i}(-1)^{i}\left[H^{i}(X, \mathcal{F})\right]$ est un multiple de la classe de la représentation régulière $k[G]$ dans l'anneau des représentations $R_{k}(G)$.

Ce résultat découle du théorème de Lefschetz, voir par exemple [15], VI, §9. Celui-ci affirme en effet que le caractère de Brauer correspondant à $\chi(G, \mathcal{F})$ s'annulle en un élément non trivial et d'ordre premier à la caractéristique du groupe $G$ lorsque l'action est sans points fixes. On note qu'on peut bien sûr déterminer de quel multiple entier il s'agit en fonction de $\mathcal{F}$, voir [1], proposition 1.2 , mais on se contente ici d'énoncés décrivant qualitativement la structure des modules galoisiens.

La proposition ci-dessus suggère le problème suivant : 
Problème 1.2

Quel est le lien entre les caractéristiques d'Euler équivariantes des $G$-faiceaux sur une $G$-variété projective $X$ et la ramification du quotient $\pi: X \rightarrow Y=X / G$ ?

En guise de première réponse, voici un résultat plus proche du théorème originel de Noether :

\section{Théorème (Nakajima 1986)}

Soit $\pi: X \rightarrow Y$ un revêtement galoisien de groupe $G$ de courbes projectives et lisses sur un corps algébriquement clos $k$. On a équivalence entre les deux propriétés :

(i) $\pi$ est modérement ramifié

(ii) Pour tout diviseur $G$-invariant $D$ sur $X$ tel que $H^{1}(X, \mathcal{L}(D))=0, H^{0}(X, \mathcal{L}(D))$ est projectif comme $k[G]$-module.

On trouve une preuve de l'implication $(i i) \Rightarrow(i)$ dans [26]. L'implication réciproque découle d'un critère de projectivité en dimension quelconque de Nakajima. Ce critère est prouvé en se ramenant au cas étale ([25]) puis en considérant des complexes de Cěch ([24]). Bien qu'également de nature qualitative, ce théorème est utile pour déterminer la structure galoisienne explicite des groupes de cohomologie.

Pour finir, voici un résultat analogue de Kani ([16], Theorem 1) :

\section{Théorème (Kani 1986)}

Soit $\pi: X \rightarrow Y$ un revêtement galoisien de groupe $G$ de courbes projectives et lisses sur un corps algébriquement clos $k$. On note $S$ un diviseur réduit sur $X$ dont le support contient le lieu de ramification de $\pi$, et $\Omega_{X}(S)$ le faisceau des différentielles sur $X$ logarithmiques le long de $S$.

Alors $\pi$ est modérement ramifié si et seulement si $H^{0}\left(X, \Omega_{X}(S)\right)$ est projectif comme $k[G]$-module.

Kani se sert de ce résultat pour déterminer la structure galoisienne du module des différentielles holomorphes sur $X$ dans le cas modéré.

\subsection{Résultats du type Riemann-Roch}

Dans cette section, on va examiner des formules donnant la structure galoisienne explicite des sections globales de $G$-faisceaux sur des variétés. On se contentera parfois d'un résultat plus faible, à savoir le calcul de la caractéristique d'Euler équivariante dans l'anneau des caractères du groupe considéré.

Toutes ces formules peuvent être considérées comme des formules de RiemannRoch à valeurs dans un tel anneau des caractères, même si elles peuvent sembler fort différentes à priori. On les regroupe par familles, suivant les outils employés pour les démontrer. 


\subsubsection{Formule de Chevalley-Weil}

En 1934 Chevalley et Weil énoncent un résultat fondateur qui détaille la structure galoisienne du module des différentielles d'ordre $f$ sur une surface de Riemann $X$ munie d'une action d'un groupe fini $G$. Pour exprimer ce résultat on note $Y$ la surface de Riemann quotient et $C_{1}, C_{2}, \ldots, C_{l}$ les points de ramification sur $Y$, $n_{\mu}$ l'indice de ramification correspondant à $C_{\mu}$ et $n$ l'ordre de $G$. Par la suite on cite [5] traduit de l'allemand avec des notations modernes :

"Nous voulons à présent déterminer combien de fois une représentation irréductible donnée $V$ de $G$ de degré $r$ est contenue dans la représentation $H^{0}\left(X, \Omega^{\otimes f}\right)$. Lorsque au dessus d'un point de ramification $C_{\mu}$ il y a $n / n_{\mu}$ points de $X$, en lequels les $n$ feuillets de $X$ se rassemblent par $n_{\mu}$, à un tour dans le sens positif autour de $C_{\mu}$ correspond une classe d'éléments conjugués d'ordre $n_{\mu}$ dans $G$, et une classe de matrices dans la représentation $V$, qui ont toutes les mêmes racines caractéristiques ; parmi ces racines, qui sont des racines $n_{\mu}$-ièmes de l'unité, la racine $e^{\frac{2 \pi i \alpha}{n_{\mu}}}$ apparaît $N_{\mu, \alpha}$ fois. Notre résultat s'énonce alors :

\section{Théorème (Chevalley-Weil 1934)}

$H^{0}\left(X, \Omega^{\otimes f}\right)$ contient $N$ fois la représentation irréductible $V$ où

$$
N=r(2 f-1)(g-1)+\sum_{\mu=1}^{l} \sum_{\alpha=0}^{n_{\mu}-1} N_{\mu, \alpha}\left((f-1)\left(1-\frac{1}{n_{\mu}}\right)+<\frac{f-1-\alpha}{n_{\mu}}>\right)+\sigma
$$

avec $\sigma=1$ quand $f=1$ et $V$ est la représentation identité (alors naturellement $N=g$ ), sinon toujours $\sigma=0 .\langle x\rangle=x-[x]$ signifie partie fractionnaire de $x$."

Pour une preuve moderne et purement algébrique on pourra consulter [35], Theorem 2. On peut brièvement analyser ce résultat dans la perspective du problème 1.2. Les nombres $N_{\mu, \alpha}$ concrétisent l'influence de la ramification sur les structures galoisiennes considérées, par exemple si l'action est libre et $f>1$ le module $H^{0}\left(X, \Omega^{\otimes f}\right)$ est $k[G]$-libre.

Ce théorème a été généralisé dans plusieurs directions. Egalement inspirés par le théorème de $G$-signature d'Atiyah et Segal, Ellingsrud et Lønsted ont démontré la version suivante ([7], Theorem 3.8). On note $n$ l'ordre de $G$, qu'on suppose premier à la caractéristique du corps de base $k$. Pour tout nombre $e$ divisant $n$, on note $\chi_{e}$ le caractère de $G$ correspondant à la multiplication par une racine primitive $e$-ième de l'unité de $k$ choisie arbitrairement. Soit de plus $\mathcal{L}$ un $G$-faisceau inversible, on suppose que pour tout point de ramification $Q$ de $Y$, admettant $P$ par antécédent par $\pi$, le groupe de décomposition $G_{P}$ de $P$ agit sur $\mathcal{L}_{P}$ par $\chi_{e_{Q}}^{r_{Q}}$, où $e_{Q}$ désigne l'indice de ramification de $Q \cdot r_{Q}$ est bien défini modulo $e_{Q}$.

\section{Théorème (Ellingsrud-Lønsted 1980)}

Soit $\pi: X \rightarrow Y$ un revêtement galoisien de groupe $G$ de courbes projectives et lisses sur un corps algébriquement clos $k$. On suppose que la caractéristique de 
$k$ ne divise pas $n$. On note $g$ le genre de $Y$. On a alors :

$\chi(G, \mathcal{L})=\left(\frac{\operatorname{deg}(\mathcal{L})}{n}+1-g-\sum_{Q}\left(1-\frac{1}{e_{Q}}\right)\right)[k[G]]+\sum_{V} \sum_{Q} \sum_{d=0}^{e_{Q}-1}<\frac{r_{Q}-1-d}{e_{Q}}>n_{d, Q, V} \cdot[V]$

où $Q$ varie parmi tous les points de ramification sur $Y$ et $V$ parmi toutes les représentations irréductibles de $G$.

Les $n_{d, Q, V}$ sont des entiers dépendants seulement de la $G$-courbe $X$, et les $r_{Q}$ sont des invariants locaux déterminés par $\mathcal{L}$ (pour des définitions précises, voir [7]). Concernant le problème 1.2, on peut faire exactement la même remarque que celle faite à propos de la formule de Chevalley-Weil, en particulier si l'action est libre le module $\chi(G, \mathcal{L})$ est $k[G]$-libre, et le terme de droite dans la formule ci-dessus doit donc s'interpréter comme une contribution de la ramification de l'action.

La formule d'Ellingsrud et Lønsted est une amélioration de celle de Chevalley et Weil de plusieurs points de vue. D'une part sa démonstration ne fait pas appel à des outils de type transcendant (alors que Chevalley et Weil utilisaient par exemple le groupe fondamental topologique de la courbe quotient privée des points de branchement) et elle est valable en caractéristique quelconque. D'autre part elle donne une formule globale dans un anneau de caractères, et ne fait pas apparaitre de décomposition en modules isotypiques (bien que sa démonstration y fasse appel). Enfin elle est vraie pour un $G$-faisceau inversible quelconque (les auteurs donnent même une formule pour un $G$-faisceau localement libre de rang quelconque) et se spécialise en la formule de Chevalley et Weil.

Ces auteurs soulignent dans l'introduction de [7] une des limites de leur approche. L'utilisation de la $K$-théorie (et en particulier l'emploi de suites exactes non scindées) impose au résultat d'être à valeurs dans l'anneau des caractères du groupe considéré. Même si cette approche se généralisait au cas non réductif (i.e. lorsque la caractéristique du corps divise l'ordre du groupe), elle déterminerait au mieux le caractère de Brauer de la représentation considerée, ce qui est insuffisant pour déterminer cette représentation à isomorphisme près. Ainsi par exemple la structure du modules des différentielles holomorphes dans le cas non réductif ne peut être connue qu'en employant d'autres méthodes. Pour une résolution par une approche aritmétique de ce problème précis, on pourra consulter [35], Theorem 2, qui donne la décomposition de ce module en modules indécomposables dans le cas d'une action d'un $p$-groupe cyclique, et qui constitue une deuxième généralisation de la formule de Chevalley et Weil. On peut de plus noter que ce problème a été résolu par Kani dans le cas de la ramification modérée (i.e. la caractéristique du corps de base ne divise pas l'ordre des stabilisateurs), voir [16] Theorem 3, mais qu'il est à notre connaissance encore ouvert dans le cas sauvage.

Une deuxième limite de la formule d'Ellingsrud et Lønsted est le caractère non explicite des entiers $n_{d, Q, V}$ (la même critique peut-être faite à propos de la 
formule de Chevalley et Weil). Un progrès dans cette direction a été accompli dans le cas de la ramification modérée par Nakajima, qui donne une formule pour le caractère de Brauer de la caractéristique équivariante d'un $G$-faisceau localement libre de rang quelconque ([25], Theorem 2). En effet spécialisée en un faisceau du type $\mathcal{L}_{X}(D)$ où $D$ est un $G$-diviseur, elle n'est exprimée qu'en fonction des coefficients de $D$ et des données d'Hurwitz (qui sont les caractères décrivant l'action des stablisateurs des points de ramification sur les espaces cotangents à la courbe en ces points).

Une troisième limite de la formule d'Ellingsrud et Lønsted est le fait qu'elle ne se spécialise pas clairement en la formule de Riemann-Roch usuelle dans le cas d'une action triviale. Cependant ces auteurs donnent une autre formule, démontrée indépendament, pour la caractéristique d'Euler équivariante du pullback d'un faisceau inversible sur le quotient ([7], Corollary 4.3), à savoir :

$$
\chi\left(G, \pi^{*} \mathcal{M}\right)=\chi\left(G, \mathcal{O}_{X}\right)+\operatorname{deg}(\mathcal{M}) \cdot[k[G]]
$$

De manière surprenante, aucune comparaison de ces deux formules n'est faite dans [7].

\subsubsection{Formule de Weil}

Dans cette section, on évoque brièvement une formule intéressante dans la perspective du problème 1.2. On suit l'exposé de Serre ([30] chapitre VI §4) de la formule de Weil.

Soit $\pi: X \rightarrow Y$ un revêtement galoisien de groupe $G$ de courbes projectives et lisses sur un corps algébriquement clos $k$. A chaque point de ramification $Q$ de $Y$ on peut associer une représentation du groupe $G$, qu'on appelle la représentation d'Artin associée à $Q$, et qu'on note $a_{Q}$. On a alors :

\section{Théorème (Weil)}

On a la relation entre caractères de représentations :

$$
H_{0}\left(X, \mathbb{Z}_{l}\right)-H_{1}\left(X, \mathbb{Z}_{l}\right)+H_{2}\left(X, \mathbb{Z}_{l}\right)=\chi(Y) \cdot k[G]-\sum_{Q \in Y} a_{Q}
$$

Il est intéressant de souligner que la définition des $a_{Q}$ fait intervenir les groupes de ramification supérieurs, ce qui montre que les structures galoisiennes peuvent être liées à la ramification sauvage de $\pi$.

D'autre part, la preuve donnée par Serre utilise une formule de Lefschetz et la formule d'Hurwitz, suivant la stratégie qui est décrite au paragraphe suivant.

\subsubsection{Formule de Lefschetz}

Dans ce paragraphe, on présente une stratégie concernant le problème du calcul des structures galoisiennes d'origine géomètrique utilisant une formule de Lef- 
schetz, plus précisement celle concernant les opérateurs périodiques. On ne se servira que d'une version simplifiée d'une formule due à Baum, Fulton et Quart $([2])$.

Soit $X$ une variété projective et lisse sur un corps algébriquement clos $k$, munie d'une action fidèle d'un groupe fini $G$. On fixe un $G$-faisceau cohérent $\mathcal{E}$. Soit $g$ un élément de $G$ différent du neutre et d'ordre premier à la caractéristique de $k$. Pour simplifier, on va supposer que les points fixes de $G$ sont isolés. Alors [15] VI $\S 9$ donne la valeur du caractère de Brauer de la caractéristique équivariante de $\mathcal{E}$ évaluée en $g$ :

$$
\chi(G, \mathcal{E})(g)=\sum_{P \in X^{g}} \frac{\operatorname{tr}\left(g, \mathcal{E}_{P}\right)}{\operatorname{det}\left(I-g_{P}\right)}
$$

où $g_{P}$ désigne l'action de $g$ sur l'espace cotangent à $X$ en $P$. L'idée est de réunir ces informations pour les différents éléments $g$ du groupe $G$. Une première limite immédiate de cette méthode est de ne pouvoir fournir qu'un caractère de Brauer. Ceci est en fait propre à l'utilisation de la $K$-théorie . Une deuxième limite plus importante est de ne fournir aucun renseignement pour la valeur de ce caractère en $g=1$.

Il semble donc qu'il faille complèter cette méthode par un calcul indépendant de $\chi(G, \mathcal{E})(1)=\chi(\mathcal{E})$, la caractéristique d'Euler usuelle. On peut à cette fin utiliser les formules de Riemann-Roch classiques, éventuellement complètées par des formules de type Hurwitz, comme au paragraphe précédent. Cependant, cette méthode semble susceptible de permettre de vérifier des résultats dont on a déjà la forme définitive, mais pas d'en démontrer de nouveaux.

\subsubsection{Formules déduites du formalisme de Riemann-Roch}

On va à présent discuter d'une autre approche du problème du calcul de la caractéristique d'Euler équivariante d'un $G$-faisceau localement libre sur une variété. Cette approche utilise le formalisme de Riemann-Roch tel qu'il est par exemple défini dans [15].

Ce problème est aisé à reformuler en termes de groupes de Grothendieck. On anticipe sur les définitions précises qui seront données dans le chapitre 2. Soit $X$ une variété projective définie sur un corps algébriquement clos $k$. Le morphisme structurel $f: X \rightarrow$ spec $k$ induit par push-forward un morphisme de groupes $f_{*}: K(G, X) \rightarrow R_{k}(G)$ qui à la classe d'un $G$-faisceau $\mathcal{E}$ localement libre sur $X$ associe la classe de sa caractéristique d'Euler équivariante $\chi(G, \mathcal{E})$. Le problème de Riemann-Roch équivariant est donc de calculer $f_{*}$.

Dans le cas non équivariant on introduit un anneau de cycles $A(X)$ et on considère le caractère de Chern $c h$ comme transformation naturelle entre les foncteurs $K($.$) et A($.$) . Le théorème de Grothendieck-Riemann-Roch exprime le comporte-$ ment du caractère de Chern vis-à-vis du push-forward. Ce théorème s'applique 
pour différents types d'anneaux de cycles, qui peuvent provenir de la théorie de l'intersection, mais qui peuvent être également de nature cohomologique, ou encore issus d'une construction de Grothendieck faisant intervenir la $\gamma$-filtration sur $K(X)$.

Dans le cas équivariant toute la difficulté réside dans le choix des cycles et la définition du caractère de Chern. Voici deux tentatives dans cette direction.

\section{Cycles à valeurs le gradué de $K(G, X)$ associé à la $\gamma$-filtration}

On résume la démarche de Köck dans [17]. Celui-ci déduit de l'existence de la $\gamma$-filtration sur $K(G, X)$ ([15], chapter III) un caractère de Chern :

$$
\operatorname{ch}: K(G, X) \rightarrow \hat{\operatorname{Gr}} K(G, X)_{\mathbb{Q}}
$$

où $\hat{G r} K(G, X)_{\mathbb{Q}}=\prod_{n \geq 0} F^{n} K(G, X)_{\mathbb{Q}} / F^{n+1} K(G, X)_{\mathbb{Q}}$ est le complèté du gradué de $K(G, X)$. Il en déduit un théorème de Grothendieck-Riemann-Roch ([17], section 5). On suit un argument de Köck pour expliquer que ce théorème spécialisé à nos besoins particuliers, ne donne pas d'information non équivariante. En effet appliqué au morphisme structurel le théorème de Köck donne une formule à valeurs dans $\hat{\operatorname{Gr}} R_{k}(G)_{\mathbb{Q}}$. Or on a le résultat suivant :

\section{Proposition 1.3}

Soit $G$ un groupe fini. On a :

$$
\operatorname{Gr} R_{k}(G)_{\mathbb{Q}} \simeq \mathbb{Q}
$$

Cette proposition découle du résultat plus général de Köck : [17] proposition 6.2. L'idée de la preuve est la suivante. On a un morphisme surjectif naturel :

$$
\operatorname{Gr} R_{k}(G)=\operatorname{Gr} K(G, \operatorname{spec}(k)) \rightarrow \operatorname{Gr} K(\operatorname{spec}(k))=\mathbb{Z}
$$

La proposition découle du fait que le noyau de ce morphisme est un groupe de torsion (il est en fait annulé par le cardinal de $G$ ). En effet, soit $\beta$ dans $\operatorname{Gr} R_{k}(G)$, comme \#G-[k[G]] est dans l'idéal d'augmentation de $R_{k}(G)$, on a $(\# G-[k[G]]) \cdot \beta=0$, mais d'autre part on a un isomorphisme $k[G] \otimes V \simeq$ $k[G]^{\oplus \operatorname{dim} V}$ pour tout $k[G]$-module $V$, ce qui donne $[k[G]] \cdot \beta=\operatorname{dim}(\beta) \cdot[k[G]]$. Donc si $\beta$ est dans le noyau du morphisme ci-dessus on a $[k[G]] \cdot \beta=0$. On en déduit que $\# G \cdot \beta=0$.

\section{Cycles associés à la théorie de l'intersection équivariante}

La théorie de l'intersection équivariante développée par Edidin et Graham ([9]) fournit des groupes de cycles équivariants construit suivant une idée de Totaro ([34]). Récemment, Edidin et Graham ont défini des classes de Chern à valeurs dans ces groupes de cycles, ce qui leur a permis d'énoncer une version équivariante du théorème de Hirzebruch-Riemann-Roch ([10], Corollary 3.1). 


\section{Chapitre 2}

\section{$G$-faisceaux}

Dans ce chapitre, on introduit la notion de $G$-faisceau qui généralise les notions de faisceau et de représentation.

\subsection{Généralités}

\subsubsection{Conventions}

Dans toute la suite, le symbole $G$ (et parfois $H, \ldots$ ) désignera un groupe fini. Tous les schémas intervenant seront supposés noethériens. Par faisceau localement libre on entendra faisceau localement libre de rang fini. Enfin, on supposera parfois implicitement, s'il n'y pas d'ambiguïté, que les morphismes intervenant commutent avec l'action du groupe $G$. Si $g$ est une bijection d'un ensemble $X$ et $f$ est une fonction sur $X, f^{g}$ désignera la fonction sur $X$ définie par $f^{g}(P)=$ $f\left(g^{-1} P\right)$.

\subsubsection{Définition}

Soit $X$ un $G$-espace, i.e. un espace topologique muni d'une action à gauche de $G$ par homéomorphismes. Pour un élément $g$ de $G$ on note encore $g$ l'homéomorphisme de $X$ correspondant.

\section{Définition 2.1}

Soit $\mathcal{F}$ un faisceau (d'ensembles, de groupes ... ) sur $X$. On appelle $G$ linéarisation de $\mathcal{F}$ la donnée d'une collection $\left(\psi_{g}\right)_{g \in G}$ de morphismes de faisceaux $\psi_{g}: g_{*} \mathcal{F} \rightarrow \mathcal{F}$ vérifiant les conditions suivantes :

(i) $\psi_{1}=\mathrm{Id}$

(ii) $\psi_{h g}=\psi_{h} \circ h_{*}\left(\psi_{g}\right)$ (condition de cocycle)

Un $G$-faisceau sur $X$ est un faisceau muni d'une $G$-linéarisation. 


\section{Remarque :}

Si $\mathcal{F}$ est un $G$-faisceau $G$ agit naturellement sur l'espace $\Gamma_{X} \mathcal{F}=H^{0}(X, \mathcal{F})$ des sections globales de $\mathcal{F}$.

La proposition suivante permet d'interpréter la définition d'un $G$-faisceau en termes d'action au sens usuel.

\section{Proposition 2.2}

Il existe une bijection naturelle entre les $G$-linéarisations de $\mathcal{F}$ et les actions par homéomorphismes de $G$ sur l'espace étalé de $\mathcal{F}$ compatibles avec l'action de $G$ sur $X$.

\section{Remarque :}

Cette compatibilité sous-entend que si $\mathcal{F}$ est un faisceau de groupes l'application $\mathcal{F}_{P} \rightarrow \mathcal{F}_{g P}$ induite par l'action sur l'espace étalé de $\mathcal{F}$ est un morphisme de groupes (et de même avec tout type de faisceau).

\section{Démonstration :}

On note Spe $\mathcal{F}$ l'espace étalé de $\mathcal{F}$. Une $G$-linéarisation induit une action naturelle sur Spe $\mathcal{F}$ : en effet chaque $\psi_{g}$ induit un morphisme $\mathcal{F}_{g^{-1} Q} \rightarrow \mathcal{F}_{Q}$, pour tout $Q$ dans $X$, et donc une application Spe $\mathcal{F} \rightarrow$ Spe $\mathcal{F}$ compatible avec l'action de $G$. La définition d'une $G$-linéarisation impose à cette application d'être une bijection. C'est de plus un homéomorphisme puisqu'elle conserve les sections, et donc la topologie de $\operatorname{Spe} \mathcal{F}$. Enfin le fait que la donnée de ces homéomorphismes définit une action résulte de l'égalité $\left(h_{*} \psi_{g}\right)_{Q}=\left(\psi_{g}\right)_{h^{-1} Q}$ pour tout $h, g$ dans $G$ et tout $Q$ dans $X$, qui est vraie car $h$ est un homéomorphisme de $X$.

Réciproquement supposons donnée une action sur Spe $\mathcal{F}$ vérifiant les conditions de la proposition. Soient $U$ un ouvert de $X, g$ dans $G$ et $s$ dans $\mathcal{F}\left(g^{-1} U\right)$. Comme $\mathcal{F}$ est un faisceau, on peut voir $s$ comme section continue de Spe $\mathcal{F} \rightarrow X$ au dessus de $g^{-1} U$. Alors l'application $P \rightarrow g\left(s_{g^{-1} P}\right)$ définit une section continue au dessus de $U$, et donc un élément de $\mathcal{F}(U)$. La stabilité par restriction étant évidente, ceci définit donc un morphisme de faisceaux $g_{*} \mathcal{F} \rightarrow \mathcal{F}$. Enfin, le fait que l'on se soit donné une action sur Spe $\mathcal{F}$ montre que la donnée de ces morphismes de faisceaux constitue une $G$-linéarisation de $\mathcal{F}$.

\subsubsection{Exemples}

D'après Grothendieck ([13] Chapitre V §5.1) : "De façon imagée, on peut dire que si $X$ est muni de certaines structures et si un faisceau $A$ sur $X$ est défini en termes structuraux, alors $A$ est un $G$-faisceau de façon naturelle si les opérations de $G$ dans $X$ sont des automorphismes." Dans ce paragraphe nous donnons quelques exemples classiques, en précisant les $G$-linéarisations. 


\section{Faisceau structurel et $G$-modules}

Soit $\left(X, \mathcal{O}_{X}\right)$ un $G$-espace annelé, i.e. un espace annelé muni d'une action de $G$. Chaque $g$ dans $G$ fournit un isomorphisme $g^{\#}: \mathcal{O}_{X} \rightarrow g_{*} \mathcal{O}_{X}$, la collection $\left(\left(g^{\#}\right)^{-1}\right)_{g \in G}$ fournit une $G$-linéarisation de $\mathcal{O}_{X}$ qui est donc muni canoniquement d'une structure de $G$-faisceau.

Avec ces mêmes hypothèses un $G$-module sur $X$ est un module $\mathcal{F}$ sur $X$ muni d'une $G$-linéarisation telle que le morphisme $\mathcal{O}_{X} \times \mathcal{F} \rightarrow \mathcal{F}$ soit un $G$-morphisme (i.e. commute avec l'action de $G$ ).

Le cadre des $G$-espace annelés est agréable, en effet il permet de traiter en même temps deux situations courantes.

Si on part d'un $G$-espace $X$, on peut le munir du faisceau constant égal à $\mathbb{Z}$ muni de l'action triviale, les $G$-modules sont alors les $G$-faisceaux de groupes abéliens. Même lorsque $X$ est muni de structures supplémentaires, ce point de vue est utile pour définir la cohomologie des $G$-faisceaux sur $X$.

D'autre part, si $X$ un $G$-schéma, i.e. un schéma muni d'une action de $G, X$ est bien sur un $G$-espace annelé, et on dispose de $G$-modules, qu'on peut supposer (semi)-cohérents, localement libres, etc.

\section{Faisceaux associés à des diviseurs invariants}

Soit $X$ un $G$-schéma intègre. Le faisceau $\mathcal{K}_{X}$ des fonctions rationelles sur $X$ est munie d'une $G$-linéarisation naturelle induite par celle de $\mathcal{O}_{X}$, qui en fait un $G$-module . (Ceci découle simplement de la propriété universelle vérifiée par le corps des fractions d'un anneau intègre).

On en déduit que $G$ agit sur le groupe $H^{0}\left(X, \mathcal{K}_{X}^{*} / \mathcal{O}_{X}^{*}\right)$, autrement dit $G$ agit sur les diviseurs de Cartier de $X$ de manière compatible avec l'addition. Plus concrètement si un diviseur de Cartier $D$ sur $X$ est représenté par une famille $\left(U_{i}, f_{i}\right)_{i \in I}$, où $\left(U_{i}\right)_{i \in I}$ est un recouvrement de $X$ et $f_{i}$ est une fonction dans $\mathcal{K}_{X}^{*}\left(U_{i}\right)$, alors pour $g$ dans $G$ le diviseur $g D$ est représenté par la famille $\left(g U_{i}, f_{i}^{g}\right)_{i \in I}$.

Pour définir la $G$-linéarisation associée à un diviseur invariant on va décrire l'action d'une façon plus fonctorielle. Pour cela rappelons le fait suivant ([14], II 6.13) : les diviseurs de Cartier sur $X$ sont en bijection avec les sous-faisceaux inversibles de $\mathcal{K}_{X}$ (i.e. avec les couples $(\mathcal{L}, \phi)$ où $\mathcal{L}$ est un faisceau inversible et $\phi: \mathcal{L} \rightarrow \mathcal{K}_{X}$ une injection, modulo les isomorphismes de faisceaux plongés). A présent si $D$ est un diviseur de Cartier sur $X$ l'injection canonique $\mathcal{L}(D) \rightarrow \mathcal{K}_{X}$ et le morphisme $g_{*}\left(\mathcal{K}_{X}\right) \rightarrow \mathcal{K}_{X}$ issu de la $G$-linéarisation de $\mathcal{K}_{X}$ définissent une injection $g_{*}(\mathcal{L}(D)) \rightarrow \mathcal{K}_{X}$, qui a son tour définit un diviseur de Cartier. Ceci définit une action sur les diviseurs de Cartier qui coïncide avec l'action naturelle définie ci-dessus, autrement dit on a un isomorphisme plongé $g_{*}(\mathcal{L}(D)) \simeq \mathcal{L}(g D)$ dont il est facile de voir qu'il est unique.

Si $g D=D$ la donnée de ces isomorphismes définit une $G$-linéarisation de $\mathcal{L}(D)$ qui fait de l'injection canonique $\mathcal{L}(D) \rightarrow \mathcal{K}_{X}$ un $G$-morphisme. 


\section{Faisceaux de différentielles}

Soit $X \rightarrow Y$ un $G$-morphisme entre $G$-schémas. Pour définir une structure de $G$-faisceau sur le faisceau des différentielles relatives $\Omega_{X / Y}$ le plus rapide est de se servir de propriétés fonctorielles : en effet $\Omega_{X / Y}=\Delta^{*}\left(\mathcal{I} / \mathcal{I}^{2}\right)$ où $\Delta$ est le morphisme diagonal $\Delta: X \rightarrow X \times_{Y} X$ et $\mathcal{I}$ le faisceau d'idéaux de $\mathcal{O}_{X \times_{Y} X}$ relatif à $\Delta$. Mais $\Delta$ étant un $G$-morphisme, $\mathcal{I}$ est naturellement muni d'une structure de $G$-faisceau, et les $G$-faisceaux étant stables par quotient et pull-back par $G$-morphisme, on en déduit que $\Omega_{X / Y}$ est lui aussi muni d'une $G$-linéarisation naturelle.

Cependant, cette méthode a l'inconvénient de ne pas être très explicite. Pour y remèdier on peut définir l'action directement sur les fibres de $\Omega_{X / Y} ;$ si $f \rightarrow f^{g}$ désigne l'action sur les fibres du faisceau structurel celle-ci s'écrit $f \mathrm{~d} u \rightarrow f^{g} \mathrm{~d} u^{g}$.

\section{Faisceaux sur un $G$-schéma affine}

Soit $A$ un anneau muni d'une action de $G$. Spec $A$ est alors un $G$-schéma et les $G$-modules sur Spec $A$ correspondent aux $A$-modules $M$ munis d'une action tordue de $G$, i.e. une action vérifiant :

$$
\forall g \in G \quad \forall m \in M \quad \forall a \in A \quad g \cdot(a m)=(g a) \cdot(g m)
$$

Une autre manière de la formuler est de dire que $M$ est un module sur l'anneau du groupe tordu $A \circ G$, i.e. le $A$-module libre sur $G$ muni de la multiplication :

$$
\forall a, a^{\prime} \in A \quad \forall g, g^{\prime} \in G \quad(a g) \cdot\left(a^{\prime} g^{\prime}\right)=a g a^{\prime} \cdot g g^{\prime}
$$

L'avantage de ce point de vue apparait lorsque $A=L$, un corps, et $G$ agit fidèlement sur $L$. Il résulte alors du lemme de Dedekind en théorie de Galois que $L \circ G$ est un anneau simple, dont le seul module simple est $L$ (voir [6]). Les $G$-faisceaux sur Spec $L$ sont classifiés par leur dimension sur $L$, ce qu'on peut retrouver de manière moins élémentaire par la théorie de la descente.

\subsection{Catégorie des $G$-faisceaux abéliens}

Soit $X$ un $G$-espace. On note $\operatorname{Ab}(G, X)$ la catégorie des $G$-faisceaux de groupes abéliens sur $X$. On vérifie qu'il s'agit d'une catégorie abélienne. Le fait suivant est bien connu :

\section{Proposition 2.3}

La catégorie $\operatorname{Ab}(G, X)$ a assez d'objects injectifs.

\section{Démonstration :}

Voir l'appendice A, théorème A.1. 
Si de plus $X$ est un $G$-espace annelé, on note $\operatorname{Mod}(G, X)$ la catégorie des $G$ modules sur $X$. Dans cette catégorie, on dispose en outre des opérations habituelles sur les $G$-modules : produit tensoriel, dual, puissances extérieure et symètrique.

\subsection{Action sur les groupes de cohomologie}

Soient $X$ un $G$-schéma noethérien et $\mathcal{F}$ un $G$-faisceau quasi-cohérent sur $X$ muni d'une $G$-linéarisation $\left(\psi_{g}\right)_{g \in G}$. Soit $g$ dans $G$, on a un morphisme induit en cohomologie $H^{i}\left(\psi_{g}\right): H^{i}\left(X, g_{*} \mathcal{F}\right) \rightarrow H^{i}(X, \mathcal{F})$, d'autre part le morphisme $g: X \rightarrow X$ étant affine on a une identification naturelle $H^{i}\left(X, g_{*} \mathcal{F}\right) \simeq H^{i}(X, \mathcal{F})$. Soit $h$ un autre élément de $G$, le morphisme $h: X \rightarrow X$ étant également affine $H^{i}\left(h_{*} \psi_{g}\right)$ s'identifie à $H^{i}\left(\psi_{g}\right)$ par l'identification ci-dessus. La condition de cocycle montre alors que la collection $\left(H^{i}\left(\psi_{g}\right)\right)_{g \in G}$ définit une action à gauche sur $H^{i}(X, \mathcal{F})$.

On peut donner une deuxième construction plus naturelle de cette action, et valable sur n'importe quel $G$-espace $X$. Au vu de la proposition 2.3 on dispose des foncteurs dérivés du foncteur des sections globales de la catégorie $\operatorname{Ab}(G, X)$ des $G$-faisceaux abéliens sur $X$ dans la catégorie $\operatorname{Ab}(G)$ des groupes abéliens avec action de $G$. Pour chaque $G$-faisceau $\mathcal{F}$ on obtient des $G$-groupes de cohomologie qu'on note par un abus très provisoire $H^{i}(X, \mathcal{F})$. Le rapport avec les groupes de cohomologie usuels est donné par la proposition suivante.

\section{Proposition 2.4}

Le foncteur d'oubli de la catégorie $\operatorname{Ab}(G)$ des groupes abéliens avec action de $G$ dans la catégorie Ab des groupes abéliens envoie les G-groupes de cohomologie sur les groupes de cohomologie usuels.

\section{Démonstration :}

Le diagramme

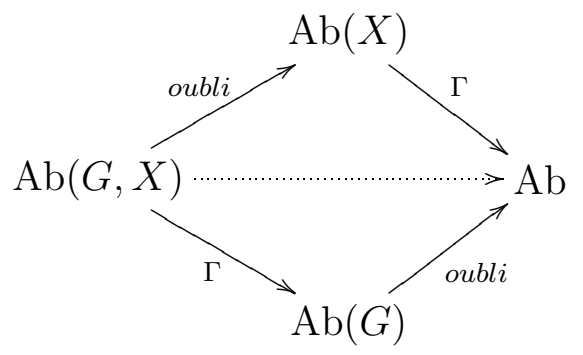

permet de décomposer le foncteur correspondant à la ligne centrale de deux manières différentes. Comme les deux foncteurs d'oubli sont exacts la proposition s'ensuit du théorème $\mathrm{A} .4$ et du fait que le foncteur d'oubli $\mathrm{Ab}(G, X) \rightarrow \operatorname{Ab}(X)$ envoie objets injectifs sur objets injectifs (voir le théorèmeA.2). 


\subsection{Formule de projection}

Soit $f: X \rightarrow Y$ un morphisme de $G$-espaces annelés. Si $\mathcal{F}$ (resp. $\mathcal{G}$ ) est un $G$ module sur $X$, alors $f_{*} \mathcal{F}$ (resp $f^{*} \mathcal{G}$ ) est naturellement muni d'une $G$-linéarisation qui en fait un $G$-module. De plus, la formule de projection est valable dans le cadre équivariant :

\section{Proposition 2.5}

Soient $X$ et $Y$ deux $G$-espaces annelés, $f: X \rightarrow Y$ un morphisme de $G$-espaces annelés, $\mathcal{F}$ un $G$-module sur $X, \mathcal{E}$ un $G$-module localement libre de rang fini sur $Y$. On a un isomorphisme de $G$-modules sur $Y$ :

$$
f_{*}\left(\mathcal{F} \otimes_{\mathcal{O}_{X}} f^{*} \mathcal{E}\right) \simeq f_{*} \mathcal{F} \otimes_{\mathcal{O}_{Y}} \mathcal{E}
$$

\section{Démonstration :}

Il est bien connu qu'un tel isomorphisme existe dans le cas non équivariant, et il est de plus naturel, il doit donc commuter à l'action de $G$. Plus précisement le morphisme canonique de $G$-modules $\mathcal{E} \rightarrow f_{*} f^{*} \mathcal{E}$ permet de construitre un morphisme de $G$-modules $f_{*} \mathcal{F} \otimes_{\mathcal{O}_{Y}} \mathcal{E} \rightarrow f_{*} \mathcal{F} \otimes_{\mathcal{O}_{Y}} f_{*} f^{*} \mathcal{E} \rightarrow f_{*}\left(\mathcal{F} \otimes_{\mathcal{O}_{X}} f^{*} \mathcal{E}\right)$. L'hypothèse que $\mathcal{E}$ est localement libre et de rang fini permet de voir que ce morphisme admet un inverse comme morphisme de modules, qui est nécessairement un morphisme de $G$-modules, d'où la proposition.

\subsection{Espace quotient}

\subsubsection{Existence}

Il est bien connu que l'existence d'un quotient d'un $G$-espace $X$ (i.e. au sens catégorique l'existence d'une flèche $\pi: X \rightarrow Y$ factorisant uniquement tout morphisme $G$-invariant) dépend de la catégorie dans laquelle on se place.

Si on se place dans la catégorie des espaces topologiques le quotient existe, l'espace sous-jacent est l'ensemble $Y$ des orbites de $X$ sous l'action de $G$, muni de la topologie dont les ouverts sont les images par $\pi: X \rightarrow Y$ des ouverts $G$-stables de $X$.

Si on se place dans la catégorie des espaces annelés on a également un quotient, dont l'espace topologique sous-jacent est l'espace topologique quotient et dont le faisceau d'anneaux est donné par

$$
\mathcal{O}_{Y}=\pi_{*}^{G}\left(\mathcal{O}_{X}\right)
$$

i.e. les sections de $\mathcal{O}_{Y}$ au-dessus d'un ouvert $V$ sont les sections de $\mathcal{O}_{X}$ au-dessus de $\pi^{-1}(V)$ invariantes sous l'action de $G$ (ce qui a un sens, puisque $\pi^{-1}(V)$ est $G$-stable).

Par contre, le quotient n'existe pas toujours dans la catégorie des schémas, d'où la définition suivante. 


\section{Définition 2.6}

Un G-schéma sera dit admissible s'il admet un quotient dans la catégorie des schémas.

\section{Remarque :}

Si l'orbite de tout point de $X$ est contenue dans un ouvert affine, $X$ est un $G$-schéma admissible (voir [23] Chapter III $§ 12$ Theorem 1).

\subsubsection{Foncteur $\pi^{*}$}

\section{$G$-linéarisationde $\pi^{*} \mathcal{G}$}

Soient $X$ un $G$-espace annelé de quotient $Y$ et $\mathcal{G}$ un $\mathcal{O}_{Y}$-module. Pour $g$ dans $G$ on a l'égalité $\pi \circ g=\pi$ et l'isomorphisme naturel $g^{*}\left(\pi^{*} \mathcal{G}\right) \simeq(\pi \circ g)^{*}(\mathcal{G})$, grâce à l'adjonction de $g^{*}$ et $g_{*}$ on en déduit des morphismes naturels $\pi^{*} \mathcal{G} \simeq$ $g_{*} \pi^{*} \mathcal{G}$. On vérifie qu'il s'agit d'isomorphismes dont les isomorphismes réciproques constituent une $G$-linéarisation de $\pi^{*} \mathcal{G} . \pi^{*} \mathcal{G}$ est donc muni naturellement d'une structure de $G$-module sur $X$.

\section{Action sur l'espace étalé de $\pi^{*} \mathcal{G}$}

On peut décrire cette structure de manière plus explicite. Comme $\pi^{*} \mathcal{G}=\mathcal{O}_{X} \otimes_{\pi^{-1} \mathcal{O}_{Y}} \pi^{-1} \mathcal{G}$ il suffit de décrire la structure de $G$-faisceau de $\pi^{-1} \mathcal{G}$. Notons $q:$ Spe $\mathcal{G} \rightarrow Y$ la projection canonique de l'espace étalé de $\mathcal{G}$ sur $Y$. Si $U$ est un ouvert de $X$, on a alors

$$
\pi^{-1} \mathcal{G}(U)=\{s: U \rightarrow \text { Spe } \mathcal{G} / s \text { continue et } q \circ s=\pi\}
$$

La $G$-linéarisation de $\pi^{-1} \mathcal{G}$ est alors donnée par :

$$
\begin{aligned}
\pi^{-1} \mathcal{G}\left(g^{-1} U\right) & \longrightarrow \pi^{-1} \mathcal{G}(U) \\
s & \longrightarrow s^{g}=s \circ g^{-1}
\end{aligned}
$$

\section{Action sur les fibres}

On a un isomorphisme naturel pour tout point $P$ de $X: \phi_{P}:\left(\pi^{-1} \mathcal{G}\right)_{P} \simeq \mathcal{G}_{\pi(P)}$. Pour tout $g$ dans $G$ on a donc des morphismes naturels :

$$
\phi_{g P}{ }^{-1} \circ \phi_{P}:\left(\pi^{-1} \mathcal{G}\right)_{P} \simeq\left(\pi^{-1} \mathcal{G}\right)_{g P}
$$

qui définissent une action de $G$ sur l'espace étalé de $\pi^{-1} \mathcal{G}$ dont on vérifie qu'elle correspond à la $G$-linéarisation des paragraphes précédents. Il est notable que l'action de $G \operatorname{sur}\left(\pi^{-1} \mathcal{G}\right)_{P}$ est induite par l'action de permutation sur $(g \cdot P)_{g \in G}$. En particuler l'action du stablisateur $G_{P}$ de $P$ sur $\left(\pi^{-1} \mathcal{G}\right)_{P}$ est triviale. 
On a donc défini deux foncteurs :

$$
\pi^{-1}, \pi^{*}: \operatorname{Mod}(G, Y) \longrightarrow \operatorname{Mod}(G, X)
$$

dont on va étudier les propriétés dans les paragraphes suivants.

\subsubsection{Foncteur $\pi_{*}^{G}$}

Dans cette section, on considère $Y$ comme $G$-espace muni de l'action triviale de $G$. Il convient de noter cependant qu'il existe des $G$-modules non triviaux sur $Y$ (i.e. des modules pour lesquels l'action sur l'espace étalé correspondant n'est pas triviale). On peut voir $\operatorname{Mod}(Y)$ comme sous-catégorie pleine de $\operatorname{Mod}(G, Y)$ en attribuant à un module sur $Y$ le même module muni de la linéarisation triviale.

Ceci étant dit on dispose de deux foncteurs :

$$
\begin{array}{cc}
\operatorname{Mod}(G, X) \stackrel{\pi_{*}}{\longrightarrow} \operatorname{Mod}(G, Y) & \operatorname{Mod}(G, Y) \longrightarrow \operatorname{Mod}(Y) \\
\mathcal{F} \longrightarrow \pi_{*} \mathcal{F} & \mathcal{G} \longrightarrow \mathcal{G}^{G}
\end{array}
$$

Le foncteur composé est noté $\pi_{*}^{G}$.

On peut noter que si l'action de $G$ sur $X$ est libre on peut construire $\pi_{*}^{G} \mathcal{F}$ comme un quotient, plus précisement on a un diagramme commutatif :

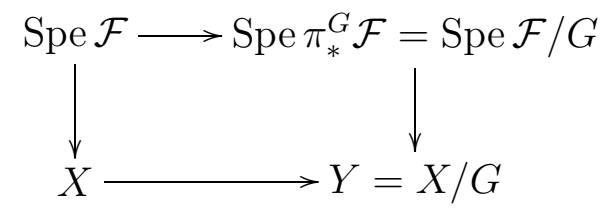

Avec cette construction, on constate facilement que si $\mathcal{F}$ est un fibré en droites, alors $\pi_{*}^{G} \mathcal{F}$ est également un fibré en droites. Cette propriété devient évidemment fausse lorsqu'on ne suppose pas l'action libre. On reviendra sur cette question (voir le théorème 2.12).

\subsubsection{Propriétés topologiques}

\section{Proposition 2.7}

Soit $X$ un $G$-espace.

(i) Pour tout objet $\mathcal{G}$ dans $\mathrm{Ab}(Y)$ on a un isomorphisme naturel

$$
\mathcal{G} \rightarrow \pi_{*}^{G} \pi^{-1} \mathcal{G}
$$

(ii) Pour tout objet $\mathcal{F}$ dans $\mathrm{Ab}(G, X)$ on a un morphisme injectif naturel $\pi^{-1} \pi_{*}^{G} \mathcal{F} \rightarrow \mathcal{F}$

(iii) Les foncteurs

$$
\begin{array}{cc}
\mathrm{Ab}(Y) \stackrel{\pi^{-1}}{\longrightarrow} \operatorname{Ab}(G, X) & \mathrm{Ab}(G, X) \stackrel{\pi_{*}^{G}}{\longrightarrow} \\
\mathcal{G} \longrightarrow \pi^{-1} \mathcal{G} & \operatorname{Ab}(Y) \\
& \mathcal{F} \longrightarrow \pi_{*}^{G} \mathcal{F}
\end{array}
$$

sont adjoints ( $\pi^{-1}$ à gauche, $\pi_{*}^{G}$ à droite). 


\section{Démonstration :}

(i) Le point de vue le plus pratique est celui des espaces étalés ; on considère les sections comme applications à valeurs dans ces espaces. Pour tout ouvert $V$ de $Y$ on a un morphisme injectif :

$$
\begin{gathered}
\mathcal{G}(V) \longrightarrow\left(\pi^{-1} \mathcal{G}\left(\pi^{-1} V\right)\right)^{G} \\
t \longrightarrow t \circ \pi
\end{gathered}
$$

La surjectivité est aisée : si $s: \pi^{-1} V \rightarrow$ Spe $\pi^{-1} \mathcal{G}=$ Spe $\mathcal{G}$ est une section continue $G$-invariante, alors d'après la propriété universelle du quotient $V=$ $\pi^{-1} V / G$, il existe une unique section continue $t: V \rightarrow \mathcal{G}$ vérifiant $t \circ \pi=s$.

(ii) Soit $U$ un ouvert de $X$ et $s$ un élément de $\pi^{-1} \pi_{*}^{G} \mathcal{F}(U)$. Comme au (i) on voit $s$ comme une application continue $s: U \rightarrow \pi_{*}^{G} \mathcal{F}$ (on confond ici $\pi_{*}^{G} \mathcal{F}$ et son espace étalé par commodité de notation) vérifiant $q \circ s=\pi, q: \pi_{*}^{G} \mathcal{F} \rightarrow Y$ étant la projection canonique, conformément au diagramme :

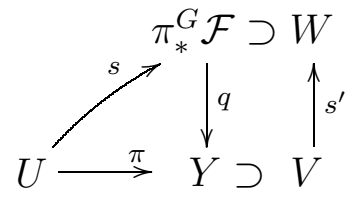

Soit $x$ dans $U$, on va traduire la continuité de $s$ en $x$. Pour cela soit $W$ un voisinage ouvert de $s(x)$ dans $\pi_{*}^{G} \mathcal{F}$. Vu la topologie sur cet espace, on peut choisir $W=s^{\prime}(V)$, où $V$ est un voisinage ouvert de $\pi(x)$ dans $Y$ et $s^{\prime}: V \rightarrow W$ une section de $q$. Comme $s$ est continue en $X$ il existe un voisinage ouvert $U^{\prime}$ de $x$ tel que $s\left(U^{\prime}\right) \subset W$. On peut bien sûr supposer également que $\pi\left(U^{\prime}\right) \subset V$. De ces deux inclusions on déduit que $s^{\prime} \circ \pi=s$, ce qui montre $s^{\prime}$ est bien définie sur l'ouvert $\pi\left(U^{\prime}\right)$, et définit donc un élément de $\pi_{*}^{G} \mathcal{F}\left(\pi U^{\prime}\right)=\left(\mathcal{F}\left(\pi^{-1} \pi U^{\prime}\right)^{G}\right.$, et donc, par restriction, un élément de $\mathcal{F}\left(U^{\prime}\right)$. Par recollement on en déduit un morphisme $\pi_{*}^{G} \mathcal{F}(\pi U) \rightarrow \mathcal{F}(U)$. La condition (locale) ci-dessus $s^{\prime} \circ \pi=s$ montre que ce morphisme est injectif.

(iii) Découle directement de (i) et (ii). On peut également dire que les foncteurs $\pi_{*}$ et $(-)^{G}$ admettent comme foncteurs adjoints à gauche respectivement le foncteur $\pi^{-1}$ et le foncteur qui à un faisceau sur $Y$ associe le $G$-faisceau trivial correspondant.

On peut se demander à quelle condition le morphisme injectif de la propriété (ii) est un isomorphisme. D'après une remarque de la section 2.5.2 une condition nécessaire est que pour tout point $P$ de $X$ l'action du stablisateur $G_{P}$ de $P$ sur $\mathcal{F}_{P}$ soit triviale. On va voir que sous certaines hypothèses cette condition est suffisante.

On peut commencer par formuler une question un peu plus forte et plus générale, à savoir : si $P$ est un point de $X$, et $Q=\pi(P)$, à quelle condition le 
morphisme injectif issu de (ii) $\left(\pi_{*}^{G} \mathcal{F}\right)_{Q} \rightarrow \mathcal{F}_{P}^{G_{P}}$ est-il un isomorphisme? Dans le cas général, on a des problèmes de recollement de sections de $\mathcal{F}$ sur des ouverts du type $U \cap g U$ où $U$ est un ouvert de $X$ et $g U$ son translaté par un élément $g$ de G. Ceci conduit Grothendieck ([13], Chapitre V, §5.3) à introduire la condition suivante :

(D) Pour tout $P$ dans $X$, le stabilisateur $G_{P}$ de $P$ est fini, et il existe un voisinage $U$ de $P$ tel que pour tout $g$ dans $G$ et non dans $G_{P}$, on ait : $U \cap g U=\emptyset$.

La première hypothèse est superflue ici puisque on suppose que $G$ est fini ; on note seulement que cette condition permet de disposer de voisinages $G$-stables de $P$. Si la condition (D) est réalisée, on a alors $\left(\pi_{*}^{G} \mathcal{F}\right)_{Q} \simeq \mathcal{F}_{P}^{G_{P}}$, et on en déduit immédiatement :

\section{Corollaire 2.8}

Soit $X$ un $G$-espace vérifiant la condition $(D)$. On a alors :

(i) Soit $\mathcal{F}$ un $G$-faisceau sur $X$. Si pour tout $P$ dans $X$ l'action du stablisateur $G_{P}$ de $P$ sur $\mathcal{F}_{P}$ est triviale on a un isomorphisme naturel $\pi^{-1} \pi_{*}^{G} \mathcal{F} \simeq \mathcal{F}$.

(ii) Si de plus $G$ agit sans points fixes les foncteurs $\pi^{-1}$ et $\pi_{*}^{G}$ établissent une équivalence de catégories entre $\operatorname{Ab}(G, X)$ et $\operatorname{Ab}(Y)$.

\subsubsection{Propriétés schématiques}

Si par exemple $X$ une $G$-variété (i.e. une variété algébrique munie d'une action d'un groupe fini $G$ ) la propriété (D) ci-dessus n'est pas vérifiée (les ouverts de la topologie de Zariski sur $X$ sont trop gros). Pour contourner cette difficulté, il est normal d'utiliser des complétions, qui servent d'analogues des ouverts trivialisants qui font défaut. On démontre alors le lemme-clé :

\section{Lemme 2.9}

Soit $X$ un $G$-schéma admissible et $\mathcal{F}$ un $G$-faisceau cohérent sur $X$. On note $\pi: X \rightarrow Y$ le quotient. Soit $P$ un point de $X$ de stabilisateur $G_{P}$ et $Q=\pi(P)$. On a alors :

$$
\left(\widehat{\pi_{*}^{G \mathcal{F}}}\right)_{Q} \simeq \widehat{\mathcal{F}}_{P}^{G_{P}}
$$

\section{Démonstration :}

Comme les morphismes de restriction sont des $G$-morphismes et $G$ est fini on a :

$$
\left(\pi_{*}^{G} \mathcal{F}\right)_{Q}=\lim _{\overrightarrow{Q \in V}}\left(\mathcal{F}\left(\pi^{-1} V\right)^{G}\right)=\left(\lim _{\overrightarrow{Q \in V}} \mathcal{F}\left(\pi^{-1} V\right)\right)^{G}=\left(\left(\pi_{*} \mathcal{F}\right)_{Q}\right)^{G}
$$

D'autre part $Y$ étant noethérien $\widehat{\mathcal{O}_{Y, Q}}$ est plat sur $\mathcal{O}_{Y, Q}$ ce qui montre que les foncteurs $\widehat{\mathcal{O}_{Y, Q}} \otimes_{\mathcal{O}_{Y, Q}}$ et $(-)^{G}$ commutent (en effet pour $B$ un anneau et $M$ un $B G$ module on a une suite exacte caractérisant $\left.M^{G}: 0 \rightarrow M^{G} \rightarrow M \rightarrow \prod_{g \in G} M\right)$. 
Il suffit donc d'étudier la complétion de $\left(\pi_{*} \mathcal{F}\right)_{Q}$ par rapport à l'idéal maximal de $\mathcal{O}_{Y, Q}$. Mais $\pi$ étant fini $\left(\widehat{\left.\pi_{*} \mathcal{O}_{X}\right)_{Q}}\right.$ est une algèbre finie sur l'anneau noethérien local complet $\widehat{\mathcal{O}_{Y, Q}}$, elle se décompose sous la forme $\left(\widehat{\pi_{*} \mathcal{O}_{X}}\right)_{Q} \simeq \prod_{P \rightarrow Q} \widehat{\mathcal{O} X, P_{X}}$. On en déduit :

$$
\widehat{\mathcal{O}_{Y, Q}} \otimes_{\mathcal{O}_{Y, Q}}\left(\pi_{*} \mathcal{F}\right)_{Q} \simeq \widehat{\mathcal{O}_{Y, Q}} \otimes_{\mathcal{O}_{Y, Q}}\left(\pi_{*} \mathcal{O}_{X}\right)_{Q} \otimes_{\left(\pi_{*} \mathcal{O}_{X}\right)_{Q}}\left(\pi_{*} \mathcal{F}\right)_{Q} \simeq \prod_{P \rightarrow Q} \widehat{\mathcal{F}_{P}}
$$

ce qui permet de conclure la démonstration de la proposition. On peut noter que seul le dernier isomorphisme utilise l'hypothèse de cohérence sur $\mathcal{F}$.

On va mettre à profit ce lemme pour montrer qu'à certaines conditions, le foncteur $\pi_{*}^{G}$ envoie un faisceau localement libre sur un faisceau localement libre.

\section{Lemme 2.10}

Soit $A / B$ une extension d'anneaux locaux noethériens. On suppose que $A$ est un $B$-module libre et est muni d'une action d'un groupe fini $H$ telle que $\# H$ est inversible dans $A$ et $B=A^{H}$. Soit de plus $M$ un $A \circ H$-module $A$-libre et de type fini sur $A$.

(i) $M^{H}$ est un $B$-module libre.

(ii) Si de plus $A$ est intègre et l'action de $H$ sur $A$ est fidèle alors $\operatorname{rg}_{B} M^{H}=$ $\operatorname{rg}_{A} M$

\section{Démonstration :}

(i) Considérons le morphisme de $B$-modules :

$$
\begin{aligned}
p_{M}: M \longrightarrow & \longrightarrow \\
\quad m & \longrightarrow \frac{1}{\# H} \sum_{h \in H} h \cdot m
\end{aligned}
$$

Son image est $M^{H}$, mais comme $p$ est une projection $M^{H}$ est facteur direct de $M$, qui est $B$-libre, donc $M^{H}$ est $B$-projectif, et comme $B$ est local, $M$ est $B$-libre.

(ii) Soit $L=\operatorname{Frac} A$ et $K=L^{G}$, on voit facilement que $K=$ Frac $B$. L'égalité recherchée découle du fait que $L \otimes_{K} K \otimes_{B} M^{G} \simeq L \otimes_{A} M$ qui est lui même une conséquence du théorème de Hilbert 90 (sous la forme $H^{1}\left(G, \mathrm{GL}_{n}(L)\right)=0$ ).

On est donc amené à poser la définition suivante.

\section{Définition 2.11}

Un $G$-schéma $X$ sera dit localement réductif si pour tout point $P$ de $X$ l'ordre du stabilisateur $G_{P}$ de $P$ est inversible dans $\mathcal{O}_{X, P}$. 


\section{Remarque :}

On n'utilisera cette définition que dans deux cas : soit lorsque $X$ est un $G$ schéma munie d'une action libre, soit lorsque $X$ est une $G$-variété munie d'une action modérée.

\section{Théorème 2.12}

Soit $X$ un $G$-schéma admissible et localement réductif, on suppose de plus le quotient $\pi: X \rightarrow Y$ plat. Si $\mathcal{F}$ est un faisceau localement libre de rang fini sur $X$ alors $\pi_{*}^{G} \mathcal{F}$ est un faisceau localement libre de même rang sur $Y$.

\section{Démonstration :}

Ce résultat découle des lemmes 2.9 et 2.10 et du fait qu'un faisceau cohérent sur un schéma noethérien est libre si et seulement si ses germes sont libres.

On peut à présent énoncer l'analogue de la proposition 2.7 :

\section{Proposition 2.13}

Soit $X$ un $G$-schéma admissible et localement réductif, de quotient $\pi: X \rightarrow Y$ plat.

(i) Pour tout faisceau localement libre de rang fini $\mathcal{G}$ sur $Y$ on a un isomorphisme naturel

$$
\mathcal{G} \rightarrow \pi_{*}^{G} \pi^{*} \mathcal{G}
$$

(ii) Pour tout $G$-faisceau cohérent $\mathcal{F}$ sur $X$ on a un morphisme injectif naturel $\pi^{*} \pi_{*}^{G} \mathcal{F} \rightarrow \mathcal{F}$

(iii) Soit $\operatorname{Proj}(G, X)$ la catégorie des $G$-faisceaux localement libres de rang fini sur $X$. Les foncteurs

$$
\begin{array}{cc}
\operatorname{Proj}(Y) \stackrel{\pi^{*}}{\longrightarrow} \operatorname{Proj}(G, X) & \operatorname{Proj}(G, X) \stackrel{\pi_{*}^{G}}{\longrightarrow} \operatorname{Proj}(Y) \\
\mathcal{G} \longrightarrow \pi^{*} \mathcal{G} & \mathcal{F} \longrightarrow \pi_{*}^{G} \mathcal{F}
\end{array}
$$

sont adjoints ( $\pi^{*}$ à gauche, $\pi_{*}^{G}$ à droite).

\section{Démonstration :}

Pour (i) et (ii) l'existence des morphismes découle de la proposition 2.7 et de l'existence du morphisme canonique $\pi^{-1} \mathcal{G} \rightarrow \pi^{*} \mathcal{G}$ pour $\mathcal{G}$ un faisceau sur $Y$. Pour montrer les propriétés locales annoncées de ces morphismes on peut les tester sur les fibres des faisceaux considérés, et même sur les complétions de ces fibres (en effet la complétion d'un anneau local noethérien est fidèlement plate).

La propriété (ii) en découle immédiatement, en appliquant le lemme 2.9.

Pour montrer (i), on note que d'après le lemme 2.9, si $Q$ est un point de $Y$ et $P$ un antécédent arbitraire par $\pi$ : 


$$
\left(\widehat{\pi_{*}^{G} \pi^{*} \mathcal{G}}\right)_{Q} \simeq\left(\widehat{\left(\pi^{*} \mathcal{G}\right)_{P}}\right)^{G_{P}} \simeq\left(\mathcal{O}_{X, P} \widehat{\otimes_{\mathcal{O}_{Y, Q}}} \mathcal{G}_{Q}\right)^{G_{P}} \simeq \mathcal{O}_{X, P}^{G_{P}} \widehat{\otimes_{\mathcal{O}_{Y, Q}}} \mathcal{G}_{Q} \simeq \widehat{\mathcal{G}_{Q}}
$$

On note que le troisième isomorphisme utilise le fait que $\mathcal{G}$ est localement libre (donc $\otimes_{\mathcal{O}_{Y, Q}} \mathcal{G}_{Q}$ commute avec $\left.(-)^{G_{P}}\right)$.

La propriété (iii) découle formellement des propriétés (i) et (ii).

\section{Corollaire 2.14}

Si l'action de $G$ sur $X$ est libre le morphisme du (ii) est un isomorphisme et les deux foncteurs $\pi^{*}$ et $\pi_{*}^{G}$ établissent une équivalence de catégories entre $\operatorname{Proj}(G, X)$ et $\operatorname{Proj}(Y)$.

\section{6 $K$-théorie équivariante}

\subsubsection{Introduction}

Afin de justifier les définitions qui suivent, et de faire le lien avec le premier chapitre, on commence par rappeler quelques idées basiques qui ont conduit à l'émergence de la $K$-théorie classique.

L'introduction des groupes de $K$-théorie en géométrie algébrique remonte à Grothendieck. Pour tout schéma noethérien $X$ celui-ci introduit un groupe abélien $K(X)$ comme étant le groupe abélien libre engendré par les classes d'isomorphismes $[\mathcal{F}]$ de faisceaux localement libres de rang fini sur $X$, modulo les relations $[\mathcal{F}]=\left[\mathcal{F}^{\prime}\right]+\left[\mathcal{F}^{\prime \prime}\right]$ s'il existe une suite exacte $0 \rightarrow \mathcal{F}^{\prime} \rightarrow \mathcal{F} \rightarrow \mathcal{F}^{\prime \prime} \rightarrow 0$.

Ces groupes de $K$-théorie ont permis à Grothendieck de démontrer une vaste généralisation de la formule de Riemann-Roch pour les variétés projectives lisses sur un corps. Afin de situer le lien, on peut supposer que $X$ est une $k$-variété projective, avec $k$ algébriquement clos. Soit $\mathcal{F}$ un faisceau localement libre de rang fini sur $X$. Alors les groupes de cohomologie de $\mathcal{F}$ sont des espaces vectoriels de dimension finie sur $k$, et sont nuls dès que leur indice dépasse la dimension de $X$, en conséquence on peut définir la caractéristique d'Euler-Poincaré $\chi(\mathcal{F})$ comme étant la somme alternée des dimensions des groupes de cohomologie de $\mathcal{F}$. Le problème de Riemann est de calculer $\chi(\mathcal{F})$ en fonction d'invariants de $X$ et de $\mathcal{F}$. Comme on le voit immédiatement grâce à la suite exacte longue de cohomologie associée à la suite exacte courte $0 \rightarrow \mathcal{F}^{\prime} \rightarrow \mathcal{F} \rightarrow \mathcal{F}^{\prime \prime} \rightarrow 0$, la caractéristique d'Euler-Poincaré est additive sur les suites exactes et définit donc un morphisme de groupes abéliens $K(X) \rightarrow \mathbb{Z}$. De manière plus fonctorielle $K(-)$ définit un foncteur (sur une catégorie à préciser) et le morphisme ci-dessus est l'image par ce foncteur du morphisme structurel $X \rightarrow \operatorname{spec} k$. On peut reformuler le problème de Riemann en disant qu'il s'agit d'étudier le comportement du foncteur $K(-)$ par push-forward.

Ce problème a été complètement résolu par Grothendieck grâce à la théorie de l'intersection, et plus précisement grâce à l'introduction du caractère de Chern 
(voir un compte-rendu dans [21]). Ce succès a provoqué un grand essor de la $K$-théorie dont le champs d'application n'est pas resté pas limité à la géométrie algébrique. Parmi les développements essentiels figure l'introduction des groupes de $K$-théorie supérieure par Quillen ([28]).

\subsubsection{Définition}

Le formalisme précédent s'applique sans modification au cas équivariant, y compris en ce qui concerne les groupes de $K$-théorie supérieure. Comme on s'intéresse ici essentiellement aux groupes de Grothendieck (i.e les groupes de $K$-théorie d'indice zéro), on se restreint à donner la définition de ceux-ci, et on renvoie à [33], [18] pour les définitions des groupes de $K$-théorie supérieure.

\section{Définition 2.15}

Soit $X$ un $G$-schéma noethérien. On désigne par $G(G, X)$ (resp. $K(G, X))$ le groupe abélien libre engendré par les classes d'isomorphismes $[\mathcal{F}]$ de $G$-faisceaux cohérents (resp. de $G$-faisceaux localement libres de rang fini) sur $X$, modulo les relations $[\mathcal{F}]=\left[\mathcal{F}^{\prime}\right]+\left[\mathcal{F}^{\prime \prime}\right]$ s'il existe une suite exacte de $G$-faisceaux $0 \rightarrow \mathcal{F}^{\prime} \rightarrow \mathcal{F} \rightarrow \mathcal{F}^{\prime \prime} \rightarrow 0$.

\section{Remarque :}

Il serait préférable de noter ces groupes $G_{0}(G, X)$ et $K_{0}(G, X)$. Pour des raisons de commodité, on omettra l'indice zéro dans les notations.

\section{Lemme 2.16}

Le produit tensoriel induit une structure d'anneau sur $K(G, X)$.

\section{Démonstration :}

Ceci résulte simplement du fait qu'un faisceau localement libre est plat.

Le morphisme naturel $K(G, X) \rightarrow G(G, X)$ induit donc une structure de $K(G, X)$ module sur $G(G, X)$. Une question naturelle est de déterminer à quelle condition ces deux groupes coïncident.

\section{Théorème 2.17}

Si $X$ est un $G$-schéma noethérien régulier et séparé le morphisme naturel $K(G, X) \rightarrow G(G, X)$ est un isomorphisme.

\section{Démonstration :}

Voir [33], Theorem 5.7 ou [18] Satz 2.1. On résume rapidement les idées de la démonstration.

Pour montrer la surjectivité on prouve que tout $G$-faisceau cohérent admet une $G$-résolution finie par des $G$-faisceaux localement libres. Pour cela on montre dans un premier temps que tout $G$-faisceau cohérent est quotient équivariant d'un $G$-faisceau localement libre, puis l'hypothèse de régularité permet d'aboutir à une résolution finie. 
L'injectivité se démontre comme dans le cas classique en prouvant que deux résolutions d'un même faisceau cohérent définissent un même élément de $K(G, X)$, en suivant par exemple la méthode de [4] théorème 2 .

\subsubsection{Propriétés fonctorielles}

Dans cette section, on étudie les propriétés fonctorielles de l'opération $(G, X) \rightarrow G(G, X)$ (resp. $(G, X) \rightarrow K(G, X))$. Son comportement fonctoriel par restriction (au sens large) de l'action à un sous groupe ne pose aucun problème (on verra ce qui se passe lorsque l'on induit l'action dans la section 2.6.5).

Les propriétés liées au changement de $G$-espace sont plus délicates. Si

$f: X \rightarrow Y$ est un $G$-morphisme arbitraire de $G$-schémas noethériens possédant de "bonnes" propriétés, $f_{*}$ et $f^{*}$ définissent par transfert de structure des foncteurs entre $\operatorname{Coh}(G, X)$ et $\operatorname{Coh}(G, Y)$ (resp. entre $\operatorname{Proj}(G, X)$ et $\operatorname{Proj}(G, Y)$ ), et il faut étudier à quelle condition ces foncteurs définissent des morphismes des groupes de Grothendieck correspondants.

\section{Contravariance}

On est dans le cas favorable où les foncteurs définis ci-dessus sont exacts, ce qui permet de définir directement les morphismes entre groupes de Grothendieck.

\section{Proposition 2.18}

(i) $(G, X) \rightarrow G(G, X)$ définit un foncteur contravariant par rapport aux morphismes de groupes et par rapport aux G-morphismes plats de schémas noethériens.

(ii) $(G, X) \rightarrow K(G, X)$ définit un foncteur contravariant par rapport aux morphismes de groupes et par rapport aux $G$-morphismes arbitraires de schémas noethériens.

\section{Démonstration :}

En ce qui concerne la contravariance par rapport aux morphismes de groupes, il suffit de noter qu'un morphisme de groupes $H \rightarrow G$ détermine par restriction une structure de $H$-schéma sur $X$, qu'une $G$-linéarisation détermine également par restriction une $H$-linéarisation, et qu'enfin que le foncteur de restriction de $\operatorname{Mod}(G, X)$ dans $\operatorname{Mod}(H, X)$ est exact.

En ce qui concerne la contravariance en la seconde variable on note tout d'abord que si $f: X \rightarrow Y$ est un $G$-morphisme arbitraire de schémas noethériens, et $\mathcal{G}$ est un $G$-faisceau cohérent sur $Y$, alors $f^{*} \mathcal{G}$ est un $G$-faisceau cohérent sur $Y$ (voir [14] chapter II Proposition 5.8 pour la cohérence de $f^{*} \mathcal{G}$, la $G$-linéarisation sur ce faisceau est obtenue par pull-back). Si de plus $\mathcal{G}$ est localement libre $f^{*} \mathcal{G}$ l'est aussi, en effet un faisceau cohérent sur un schéma noethérien est localement libre si ses fibres sont libres, mais si $P$ est un point de $X$ et $Q=f(P)$ on a : 
$\left(f^{*} \mathcal{G}\right)_{P} \simeq \mathcal{O}_{X, P} \otimes_{\mathcal{O}_{Y, Q}} \mathcal{G}_{Q}$. Enfin on note que le foncteur $f^{*}$ est exact dans les deux cas, en effet si $0 \rightarrow \mathcal{G}^{\prime} \rightarrow \mathcal{G} \rightarrow \mathcal{G}^{\prime \prime} \rightarrow 0$ est une suite exacte de $G$-modules cohérents sur $Y$, l'obstruction locale en $P$ à l'exactitude de $0 \rightarrow f^{*} \mathcal{G}^{\prime} \rightarrow f^{*} \mathcal{G} \rightarrow f^{*} \mathcal{G}^{\prime \prime} \rightarrow 0$ est $\operatorname{Tor}_{1}^{\mathcal{O}_{Y, Q}}\left(\mathcal{O}_{X, P}, \mathcal{G}_{Q}^{\prime \prime}\right)$; elle est nulle dans le premier cas car $\mathcal{O}_{X, P}$ est plat sur $\mathcal{O}_{Y, Q}$, elle est nulle dans le deuxième cas car $\mathcal{G}_{Q}^{\prime \prime}$ est libre sur $\mathcal{O}_{Y, Q}$.

\section{Covariance}

Les propriétés de covariance des groupes de Grothendieck sont à la fois plus délicates et plus intéressantes. Ceci vient en partie du fait que le foncteur image direct est rarement exact. On commence par une des rares situations où c'est quand même le cas.

\section{Proposition 2.19}

$(G, X) \rightarrow G(G, X)$ définit un foncteur covariant par rapport aux $G$-morphismes finis de schémas noethériens.

\section{Démonstration :}

Si $f: X \rightarrow Y$ est un $G$-morphisme fini de schémas noethériens, et $\mathcal{F}$ est un $G$ faisceau sur $X$, alors $f_{*} \mathcal{F}$ est un $G$-faisceau cohérent ([14] Chapter II ex 5.5 pour la cohérence, la $G$-linéarisation est obtenue par push-forward), de plus le foncteur $f_{*}$ est exact, comme on peut le voir en localisant et en complètant comme au lemme 2.9 .

\section{Remarque :}

La proposition s'applique en particulier au cas où $f$ est une immersion fermée.

Cette proposition étant insuffisante, on donne la généralisation suivante. Le défaut d'exactitude du foncteur image directe est compensé en faisant intervenir les foncteurs dérivés.

\section{Proposition 2.20}

$(G, X) \rightarrow G(G, X)$ définit un foncteur covariant par rapport aux $G$-morphismes propres de schémas noethériens.

\section{Démonstration :}

Si $f: X \rightarrow Y$ est un $G$-morphisme propre de schémas noethériens, et $\mathcal{F}$ est un $G$-faisceau sur $X$, alors les $R^{i} f_{*}(\mathcal{F})$ sont des faisceaux cohérents (EGA III 3.2.1 ou [4] théorème 1) qu'on peut munir de $G$-linéarisations par transfert de structure. D'autre part $R^{i} f_{*}=0$ pour $i$ assez grand (EGA III 1.4.12), on peut donc poser :

$$
f_{*}([\mathcal{F}])=\sum_{i}(-1)^{i}\left[R^{i} f_{*}(\mathcal{F})\right]
$$


La suite exacte longue de cohomologie associée au foncteur $f_{*}$ assure que $G(G, f)$ est bien défini et additif.

\section{Remarque :}

Le comportement du foncteur $(G, X) \rightarrow K(G, X)$ par image directe est encore plus délicat, une difficulté supplémentaire apparaissant dans le fait que l'image directe d'un faisceau localement libre n'est pas localement libre (cette difficulté existe déjà dans la théorie non équivariante, voir SGA 6 exposé 0$)$. On peut toutefois définir un morphisme $f_{*}: K(G, X) \rightarrow K(G, Y)$ lorsque $f: X \rightarrow Y$ est un morphisme projectif localement d'intersection complète (i.e. qui se décompose en une immersion fermée suivie d'une projection élémentaire, voir [18] §2). On peut également noter qu'avec cette définition $f_{*}$ devient fonctoriel pour les morphismes projectifs localement d'intersection complète. On donne ci-dessous une propriété plus faible (proposition 2.22), qui suffira cependant par la suite.

\subsubsection{Caractéristique d'Euler-Poincaré équivariante}

On peut à présent définir rigoureusement la caractéristique d'Euler-Poincaré équivariante dont on a parlé au premier chapitre :

\section{Définition 2.21}

Soient $k$ un corps algébriquement clos sur lequel $G$ agit trivialement et $X$ un $G$ schéma noethérien tel que le morphisme structurel $s: X \rightarrow \operatorname{spec} k$ soit propre et équivariant. Si $\mathcal{F}$ est un $G$-faisceau cohérent sur $X$ on définit sa caractéristique d'Euler-Poincaré équivariante comme étant le module virtuel de $R_{k}(G)$ :

$$
\chi(G, \mathcal{F}):=s_{*}([\mathcal{F}])
$$

La définition de la caractéristique d'Euler-Poincaré équivariante suggère qu'elle a un bon comportement par image directe. La proposition suivante souligne le fait que c'est bien le cas avec les immersions fermées.

\section{Proposition 2.22}

On considère le diagramme suivant :

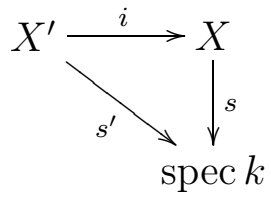

où $X, X^{\prime}$ sont deux schémas noethériens, $i$ est une $G$-immersion fermée, $s$ et $s^{\prime}$ sont deux $G$-morphismes propres, et le diagramme commute. Alors pour tout $G$-faisceau cohérent $\mathcal{F}$ sur $X^{\prime}$ on a : $\chi\left(G, i_{*} \mathcal{F}\right)=\chi(G, \mathcal{F})$. 


\section{Démonstration :}

Le diagramme commutatif ci-dessus induit un diagramme commutatif de foncteurs :

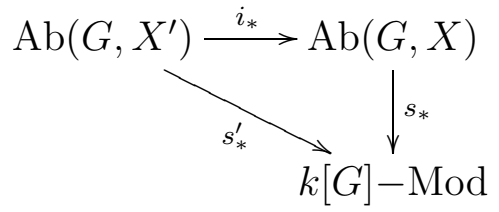

On conclue grâce au théorème A.4 et au fait que le foncteur $i_{*}$ est exact.

\subsubsection{Induction}

Afin d'étudier le comportement des groupes de $K$-théorie par extension de l'action, on présente la notion de $G$-faisceau induit, suivant une construction analogue à celle des représentations induites.

\section{Espace induit}

Soit $H$ un sous-groupe de $G$, et $X$ un $H$-espace. On munit $G \times X$ d'une action à gauche de $H$ définie par :

$$
\begin{gathered}
H \times G \times X \longrightarrow G \times X \\
\quad(h, g, x) \longrightarrow\left(g h^{-1}, h x\right)
\end{gathered}
$$

Suivant [33] on note $G \times{ }^{H} X$ le quotient. On note de plus que l'action de $G$ sur $G \times X$ définie par :

$$
\begin{array}{r}
G \times G \times X \longrightarrow G \times X \\
\left(g^{\prime}, g, x\right) \longrightarrow\left(g^{\prime} g, x\right)
\end{array}
$$

commute avec l'action de $H$ définie ci-dessus, en conséquence $G \times{ }^{H} X$ est muni d'une action de $G$. Par restriction de l'action $H$ agit sur $G \times{ }^{H} X$, cette action prolongeant l'action initiale de $H$ sur $X$, plus précisement les morphismes naturels : $X \simeq H \times{ }^{H} X \rightarrow G \times{ }^{H} X$ sont des morphismes de $H$-espaces. On en déduit que tout $G$-faisceau sur $G \times{ }^{H} X$ détermine un $H$-faisceau sur $X$ (par restriction de l'action de $G$ à $H$, puis pull-back par le $H$-morphisme $\left.X \rightarrow G \times{ }^{H} X\right)$.

\section{$G$-faisceau induit}

On va définir une procédure dans le sens inverse. Soit donc $\mathcal{F}$ un $H$-faisceau sur $X$. Du point de vue des espaces étalés on a un morphisme de $H$-espaces Spe $\mathcal{F} \rightarrow X$, la construction précédente $G \times{ }^{H}$ étant fonctorielle en le $H$-espace $X$ on obtient un $G$-morphisme $G \times{ }^{H}$ Spe $\mathcal{F} \rightarrow G \times{ }^{H} X$, qui à son tour définit un $G$-faisceau $\mathcal{F}^{\prime}$ sur $G \times{ }^{H} X$.

\section{Définition 2.23}

Le $G$-faisceau $\mathcal{F}^{\prime}$ sera appelé faisceau induit par $\mathcal{F}$ de $X$ à $G \times{ }^{H} X$ et sera noté $\operatorname{Ind}_{H}^{G} \mathcal{F}$. 
Il peut être pratique de décrire explicitement ce faisceau. On procède comme dans le cas des représentations. Pour ce faire on note $\left\{\rho_{i}, 1 \leq i \leq m\right\}$ un système de représentants de $G \backslash H$, i.e. $G=\bigsqcup_{i=1}^{n} \rho_{i} H$. Par commodité on impose $\rho_{1}=1$. On a alors une description explicite du $G$-espace $G \times{ }^{H} X: G \times{ }^{H} X \simeq \bigsqcup_{i=1}^{n} \rho_{i} X$. Ceci montre clairement que si $X$ est un $H$-schéma $G \times{ }^{H} X$ est naturellement un $G$-schéma. Si $\mathcal{F}$ est un $H$-faisceau sur $X$ on a alors :

$$
\operatorname{Ind}_{H}^{G} \mathcal{F}=\bigoplus_{i=1}^{n} \rho_{i *} \mathcal{F}
$$

Les opérations de restriction et d'induction définissent des quasi-isomorphismes réciproques entre la catégorie des $H$-faisceaux sur $X$ et la catégorie des $G$ faisceaux sur $G \times{ }^{H} X$, qui conservent le caractère cohérent ou localement libre des faisceaux lorsque $X$ est un $H$-schéma. On en déduit :

\section{Proposition 2.24}

Soit $X$ un $H$-schéma. On a des isomorphismes naturels :

$$
\begin{aligned}
G(H, X) & \simeq G\left(G, G \times{ }^{H} X\right) \\
K(H, X) & \simeq K\left(G, G \times{ }^{H} X\right)
\end{aligned}
$$

\section{Démonstration :}

Voir [33], Proposition 6.2.

\subsubsection{Suite exacte de localisation}

Les faisceaux de torsion jouent un rôle important en $K$-théorie, ils permettent en effet de nombreux dévissages, par exemple en ce qui concerne le problème du calcul de caractéristiques d'Euler, ou problème de Riemann. La suite exacte suivante, dite de localisation, est un outil de base dans l'étude de ces faisceaux, elle permet de comparer le groupe de $K$-théorie d'un schéma à celui d'un sousschéma fermé. Ici, la $K$-théorie équivariante ne se distingue pas de la $K$-théorie classique.

\section{Théorème 2.25}

Soit $X$ un $G$-schéma noethérien, $j: U \rightarrow X$ une $G$-immersion ouverte, $i: C \rightarrow$ $X$ une $G$-immersion fermée de support $X-U$. La suite suivante est exacte :

$$
G(G, C) \stackrel{i_{*}}{\longrightarrow} G(G, X) \stackrel{j^{*}}{\longrightarrow} G(G, U) \longrightarrow 0
$$

\section{Démonstration :}

La démonstration est exactement la même que dans le cas non équivariant, voir [4], §8, Proposition 7 ou [14], exercice 6.10. Pour la surjectivité de $j^{*}$ voir [33] Corollary 2.4. L'idée essentielle pour montrer l'exactitude en $G(G, X)$ est la 
suivante : si $\mathcal{F}$ est un faisceau sur $X$ à support dans $C$, alors il existe une filtration :

$$
0=\mathcal{F}_{n} \subseteq \mathcal{F}_{n-1} \subseteq \cdots \subseteq \mathcal{F}_{1} \subseteq \mathcal{F}_{0}=\mathcal{F}
$$

telle que pour tout $i$ le faisceau $\mathcal{F}_{i} / \mathcal{F}_{i+1}$ s'écrive $i_{*}\left(\mathcal{G}_{i}\right)$, pour un $G$-faisceau cohérent $\mathcal{G}_{i}$ sur $C$. En effet si $\mathcal{I}$ est le faisceau d'idéaux définissant $C$ il suffit de prendre $\mathcal{F}_{i}=\mathcal{I}^{\otimes i} \otimes_{\mathcal{O}_{X}} \mathcal{F}$.

\section{Remarque :}

La $K$-théorie supérieure permet de mesurer le défaut d'injectivité de la suite exacte de localisation, et fournit une suite exacte longue :

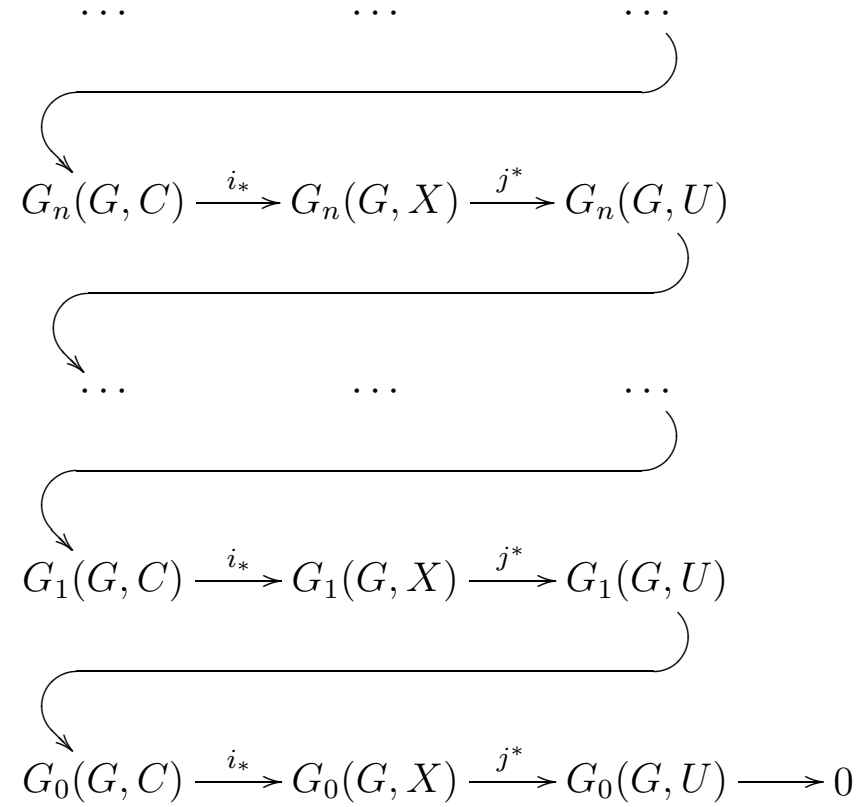

Pour une définition des groupes de $K$-théorie équivariante supérieurs, et une preuve de l'exactitude de la suite ci-dessus on renvoie à [33]. Ces notions ne seront pas indispensables par la suite, la suite exacte de localisation étant outil suffisant.

\subsubsection{Groupe de Grothendieck d'un schéma muni d'une action libre}

Lemme 2.26

Soit $X$ un $G$-schéma. On note $\theta: G \times X \rightarrow X$ l'action et $p: G \times X \rightarrow X$ la projection $(g, x) \rightarrow x$. Soit $\mathcal{F}$ un faisceau sur $X$. La donnée d'une $G$-linéarisation de $\mathcal{F}$ équivaut à la donnée d'un isomorphisme sur $G \times X$

$$
\phi: \theta^{*} \mathcal{F} \simeq p^{*} \mathcal{F}
$$


vérifiant la condition de cocycle sur $G \times G \times X$ :

$$
\left(p_{23}^{*}\right) \phi \circ\left((1 \times \theta)^{*} \phi\right)=(m \times 1)^{*} \phi
$$

où $m: G \times G \rightarrow G$ est la multiplication dans $G$.

\section{Démonstration :}

Voir [33].

Théorème 2.27

Soit $X$ un $G$-schéma admissible muni d'une action libre (i.e. les groupes d'inertie sont triviaux). Si on note $\pi: X \rightarrow Y=X / G$ le quotient on a un isomorphisme :

$$
K(G, X) \simeq K(Y)
$$

\section{Démonstration :}

Soit $\mathcal{F}$ un $G$-faisceau localement libre de rang fini sur $X$. D'après SGA1 exposé $\mathrm{V}$ proposition $2.6 \pi: X \rightarrow Y$ est galoisien étale, en particulier $\pi$ est fidèlement plat et quasi-compact, et $G \times X \simeq X \times_{Y} X$. Par cet isomorphisme la $G$-linéarisation de $\mathcal{F}$ s'identifie grâce au lemme 2.26 à une donnée de descente pour $\mathcal{F}$ relativement à $\pi$. La théorie de la descente affirme qu'il existe un faisceau $\mathcal{G}$ tel que $\mathcal{F}=\pi^{*} \mathcal{G}$ ; $\pi$ étant fidèlement plat $\mathcal{G}$ est localement libre de rang fini sur $Y$. En utilisant le lemme 2.13 (i), on voit que ceci implique que $\pi^{*} \pi_{*}^{G} \mathcal{F} \simeq \mathcal{F}$. On en déduit que $\pi^{*}$ induit une équivalence de catégories entre $\operatorname{Proj}(Y)$ et $\operatorname{Proj}(G, X)$, d'où le théorème.

\subsubsection{Groupe de Grothendieck d'un $G$-schéma admissible et groupe de Grothendieck du quotient}

Soit $X$ un $G$-schéma admissible. Au vu du paragraphe précédent, on peut se demander si le morphisme $\pi^{*}: K(Y) \rightarrow K(G, X)$ est toujours injectif. La proposition suivante affirme que c'est le cas si $X$ est localement réductif. On peut même être plus précis :

\section{Proposition 2.28}

Soit $X$ un $G$-schéma admissible localement réductif tel que le quotient $\pi: X \rightarrow$ $Y=X / G$ soit plat. Le morphisme de groupes abéliens $\pi^{*}: K(Y) \rightarrow K(G, X)$ fait de $K(Y)$ un facteur direct de $K(G, X)$.

\section{Démonstration :}

Il suffit de montrer que le foncteur $\pi_{*}^{G}$ induit un morphisme $\pi_{*}^{G}: K(G, X) \rightarrow$ $K(Y)$ tel que $\pi_{*}^{G} \circ \pi^{*}=\operatorname{Id}_{K(Y)}$, ce qui résulte du lemme 2.13 (i) et du lemme suivant. 
Lemme 2.29

Soit $X$ un $G$-schéma comme dans la proposition ci-dessus.

Le foncteur $\pi_{*}^{G}: \operatorname{Proj}(G, X) \rightarrow \operatorname{Proj}(Y)$ est exact.

\section{Démonstration :}

Ce lemme est lui même une conséquence du lemme 2.9 et de la version fonctorielle suivante du lemme 2.10 .

Lemme 2.30

Soit $A / B$ une extension d'anneaux locaux noethériens. On suppose que $A$ est un $B$-module libre et est muni d'une action d'un groupe fini $H$ telle que \#H est inversible dans $A$ et $B=A^{H}$. Soit de plus $0 \rightarrow M^{\prime} \rightarrow M \rightarrow M^{\prime \prime} \rightarrow 0$ une suite exacte de $A \circ H$-modules $A$-libres et de type fini sur $A$. Alors la suite $0 \rightarrow M^{\prime H} \rightarrow M^{H} \rightarrow M^{\prime \prime H} \rightarrow 0$ est une suite exacte de $B$-modules libres.

\section{Démonstration :}

On note $C$ le complexe de $B$-modules $0 \rightarrow M^{\prime} \rightarrow M \rightarrow M^{\prime \prime} \rightarrow 0$ obtenu par restriction des scalaires. Avec les notations de la démonstration du lemme 2.10, l'opération de moyenne fournit un endomorphisme du complexe $C: p_{C}=$ $\left(p_{M}^{\prime}, p_{M}, p_{M}^{\prime \prime}\right)$. Le fait que $p_{C}$ soit une projection montre que le complexe $C^{H}$ : $0 \rightarrow M^{\prime H} \rightarrow M^{H} \rightarrow M^{\prime \prime H} \rightarrow 0$ est facteur direct de $C$, donc son homologie est facteur direct de celle de $C$, qui est nulle par hypothèse, donc $C^{H}$ est exact.

\section{Remarque :}

Si on compare le théorème de la section 2.6.7 à la proposition 2.28 on constate que le défaut de surjectivité du morphisme $\pi^{*}: K(Y) \rightarrow K(G, X)$ est lié à l'existence de points fixes pour l'action. Un des buts de la suite de ce travail est de préciser ce lien.

\subsubsection{Groupe de Grothendieck d'un k-schéma muni d'une action triviale}

Théorème 2.31

Soient $k$ un corps algébriquement clos de caractéristique ne divisant pas l'ordre de $G$ et $X$ un $k$-schéma noethérien, tous deux munis de l'action triviale de $G$. On a un isomorphisme naturel :

$$
K(G, X) \simeq K(X) \otimes_{\mathbb{Z}} R_{k}(G)
$$

\section{Démonstration :}

Si $V$ est un $k[G]$-module, on notera $\mathbf{V}$ le $G$-faisceau :

$\mathbf{V}=\mathcal{O}_{X} \otimes_{k} V$. On a un morphisme naturel :

$$
\begin{aligned}
K(X) \otimes_{\mathbb{Z}} R_{k}(G) & \longrightarrow K(G, X) \\
{[\mathcal{F}] \otimes[V] \longrightarrow } & {\left[\mathcal{F} \otimes_{\mathcal{O}_{X}} \mathbf{V}\right] }
\end{aligned}
$$


Pour définir un morphisme réciproque, on note d'après le lemme 2.10 que si $\mathcal{E}$ et $\mathcal{F}$ sont deux $G$-faisceaux localement libres sur $X$, alors $\mathcal{H o m}_{G}(\mathcal{E}, \mathcal{F}):=\mathcal{H o m}(\mathcal{E}, \mathcal{F})^{G}$ est un faisceau localement libre sur $X$, et on peut donc définir :

$$
\begin{aligned}
& K(G, X) \longrightarrow K(X) \otimes_{\mathbb{Z}} R_{k}(G) \\
& \quad[\mathcal{F}] \longrightarrow \sum_{V \in \hat{G}}\left[\mathcal{H o m}_{G}(\mathbf{V}, \mathcal{F})\right] \otimes[V]
\end{aligned}
$$

Pour vérifier que ces deux morphismes sont réciproques l'un de l'autre, on remarque que si $\mathcal{F}$ est un $G$-faisceau localement libre sur $X$, on a un morphisme naturel :

$$
\sum_{V \in \hat{G}} \mathcal{H o m}_{G}(\mathbf{V}, \mathcal{F}) \otimes V \rightarrow \mathcal{F}
$$

Il est facile de voir que ce morphisme est un isomorphisme, en effet il suffit de le montrer au niveau des germes $\mathcal{F}_{P}$ pour tout point $P$, et même d'après le lemme de Nakayama au niveau des fibres $\mathcal{F}_{P} \otimes_{\mathcal{O}_{X, P}} k$. Mais le fait qu'on ait un isomorphisme au niveau des fibres résulte du fait que $k[G]$ est semi-simple, et donc que l'égalité ci-dessus est vraie pour les représentations.

\section{Exemple :}

On prend comme espace topologique un point $*=\operatorname{Spec} k$, le théorème ci-dessus donne $K(G, *)=R_{k}(G)$. Cette égalité est une des justifications de la notion de cycle à coefficients équivariants, qui sera developpée au quatrième chapitre.

\subsection{Groupe de Picard équivariant}

Le problème posé dans le paragraphe 2.6.7, à savoir formuler, en termes des points fixes de l'action, l'obstruction pour un $G$-faisceau à être un $G$-faisceau tiré par $X \rightarrow Y=X / G$, admet une réponse simple et générale lorsque l'on restreint le problème aux faisceaux inversibles.

\subsubsection{Introduction}

Par analogie avec le cas classique on introduit le groupe de Picard équivariant :

\section{Définition 2.32}

Soit $X$ un $G$-schéma. Le groupe de Picard équivariant de $X$, noté $\operatorname{Pic}_{G} X$, est le groupe des classes de $G$-isomorphismes de $G$-faisceaux sur $X$, muni du produit induit par le produit tensoriel.

Si $X$ admet un quotient $\pi: X \rightarrow Y=X / G$, le pullback induit un morphisme de groupes $\pi^{*}: \operatorname{Pic} Y \rightarrow \operatorname{Pic}_{G} X$ (si $X$ est localement réductif et $\pi$ est plat la proposition 2.13 (i) montre que $\pi^{*}$ est injectif) dont on va déterminer le conoyau. 
La cohomologie équivariante (voir le paragraphe suivant) est un outil utile à l'étude de ce problème, et du groupe de Picard équivariant en général, vu l'isomorphisme :

$$
\operatorname{Pic}_{G} X \simeq H^{1}\left(X, G, \mathcal{O}_{X}^{*}\right)
$$

Pour établir cet isomorphisme dans le cas où $X$ est un $G$-schéma intègre, il est utile d'introduire un certain groupe de classes de diviseurs de Cartier $G$ invariants :

\section{Définition 2.33}

Soit $X$ un $G$-schéma admissible, de quotient $X \rightarrow Y=X / G$. On note $\mathcal{K}_{X}$ (resp. $\mathcal{K}_{Y}$ ) le faisceau des quotients totaux de $\mathcal{O}_{X}$ (resp. $\mathcal{O}_{Y}$ ). Le groupe des diviseurs de Cartier $G$-invariants sur $X$, noté $\mathrm{CaCl}_{G} X$, est défini par :

$$
\mathrm{CaCl}_{G} X=\frac{H^{0}\left(X, \mathcal{K}_{X}^{*} / \mathcal{O}_{X}^{*}\right)^{G}}{\pi^{*}\left(H^{0}\left(Y, \mathcal{K}_{Y}^{*}\right)\right)}
$$

On peut à présent énoncer le résultat principal de cette section :

\section{Théorème 2.34}

Soit $X$ un $G$-schéma admissible, de quotient $X \rightarrow Y=X / G$. On suppose que $X$ est intègre et que l'action de $G$ sur $X$ est fidèle. Alors les trois groupes $\operatorname{Pic}_{G} X$, $H^{1}\left(X, G, \mathcal{O}_{X}^{*}\right)$ et $\mathrm{CaCl}_{G} X$ sont canoniquement isomorphes.

La preuve sera donnée au paragraphe 2.7.3.

\subsubsection{Cohomologie équivariante}

\section{Définition}

Soit $X$ un $G$-espace. Grothendieck a remarqué qu'on pouvait utiliser le formalisme des foncteurs dérivés pour définir une cohomologie propre à la situation équivariante. Plus précisement, le fait que la catégorie $\operatorname{Ab}(G, X)$ ait assez d'injectifs permet de poser la définition suivante :

\section{Définition 2.35}

On note $R^{q} \pi_{*}^{G}$ (respectivement $H^{q}(X, G, \cdot)$ ) les foncteurs dérivés du foncteur $\pi_{*}^{G}: \operatorname{Ab}(G, X) \rightarrow \operatorname{Ab}(Y)$ (respectivement du foncteur $\Gamma_{X}(\cdot)^{G}: \operatorname{Ab}(G, X) \rightarrow \mathrm{Ab}$ ).

\section{Suites spectrales fondamentales}

Le foncteur $\Gamma_{X}(\cdot)^{G}$ se décompose naturellement de deux manières :

$\Gamma_{X}(\cdot)^{G}=(\cdot)^{G} \circ \Gamma_{X}(\cdot)=\Gamma_{Y}(\cdot) \circ \pi_{*}^{G}(\cdot)$ et donne ainsi naissance à deux suites spectrales. 


\section{Théorème 2.36}

([13], Chapitre V, Théorème 5.2.1)

Soit $\mathcal{F}$ un $G$-faisceau abélien sur le $G$-espace $X$. Les deux suites spectrales de termes initiaux

$$
\begin{gathered}
H^{p}\left(G, H^{q}(X, \mathcal{F})\right) \\
H^{p}\left(Y, R^{q} \pi_{*}^{G} \mathcal{F}\right)
\end{gathered}
$$

convergent et aboutissent à $H^{n}(X, G, F)$.

En particulier, on a deux suites exactes à cinq termes :

$0 \rightarrow H^{1}\left(G, \Gamma_{X}(\mathcal{F})\right) \rightarrow H^{1}(X, G, \mathcal{F}) \rightarrow H^{1}(X, \mathcal{F})^{G} \rightarrow H^{2}\left(G, \Gamma_{X} \mathcal{F}\right) \rightarrow H^{2}(X, G, \mathcal{F})$

$0 \rightarrow H^{1}\left(Y, \pi_{*}^{G} \mathcal{F}\right) \rightarrow H^{1}(X, G, \mathcal{F}) \rightarrow \Gamma_{Y}\left(R^{1} \pi_{*}^{G} \mathcal{F}\right) \rightarrow H^{2}\left(Y, \pi_{*}^{G} \mathcal{F}\right) \rightarrow H^{2}(X, G, \mathcal{F})$

\section{Calcul de la cohomologie équivariante}

Voir [13] ou [22] pour une version moderne.

\subsubsection{Preuve du théorème 2.34}

Isomorphisme entre $H^{1}\left(X, G, \mathcal{O}_{X}^{*}\right)$ et $\mathrm{CaCl}_{G} X$

L'isomorphisme entre ces deux groupes découle directement de la suite exacte longue de cohomologie équivariante associée à la suite exacte courte de $G$-faisceaux sur $X: 0 \rightarrow \mathcal{O}_{X}^{*} \rightarrow \mathcal{K}_{X}^{*} \rightarrow \mathcal{K}_{X}^{*} / \mathcal{O}_{X}^{*} \rightarrow 0$ et du lemme suivant :

\section{Lemme 2.37}

Si $X$ un $G$-schéma intègre, et si l'action de $G$ sur $X$ est fidèle, alors $H^{1}\left(X, G, \mathcal{K}_{X}^{*}\right)=0$

\section{Démonstration :}

Comme $\mathcal{K}_{X}^{*}$ est un faisceau constant, $H^{1}\left(X, \mathcal{K}_{X}^{*}\right)=0$, et la première suite spectrale fondamentale donne : $H^{1}\left(X, G, \mathcal{K}_{X}^{*}\right)=H^{1}\left(G, H^{0}\left(X, \mathcal{K}_{X}^{*}\right)\right)$, mais ce dernier groupe est nul, d'après le théorème 90 de Hilbert.

\section{Isomorphisme entre $\mathrm{CaCl}_{G} X$ et $\mathrm{Pic}_{G} X$}

On a vu au paragraphe 2.1.3 qu'à un diviseur invariant sur $X$ était naturellement associé un $G$-faisceau inversible, on définit ainsi un morphisme :

$$
H^{0}\left(X, \mathcal{K}_{X}^{*} / \mathcal{O}_{X}^{*}\right)^{G} \rightarrow \operatorname{Pic}_{G}(X)
$$

Comme de plus $\pi^{*} \mathcal{O}_{Y} \simeq \mathcal{O}_{X}$ ce morphisme s'annule sur $\pi^{*}\left(H^{0}\left(Y, \mathcal{K}_{Y}^{*}\right)\right)$, et définit par passage au quotient un morphisme de groupes :

$$
\mathrm{CaCl}_{G}(X) \rightarrow \operatorname{Pic}_{G}(X)
$$


Montrer l'injectivité de ce morphisme est aisé. En effet soit $D$ un diviseur de Cartier sur $X$ dans son noyau. Des $G$-morphismes $\mathcal{O}_{X} \simeq \mathcal{L}(D)$ et $\mathcal{L}(D) \rightarrow \mathcal{K}_{X}$ on déduit un $G$-morphisme :

$$
H^{0}\left(X, \mathcal{O}_{X}\right) \simeq H^{0}(X, \mathcal{L}(D)) \rightarrow H^{0}\left(X, \mathcal{K}_{X}\right)
$$

Ce morphisme envoie 1 sur une fonction $f$ telle que $(f)=D$, comme de plus 1 est $G$-invariant $f$ est dans $H^{0}\left(X, \mathcal{K}_{X}\right)^{G}=\pi^{*}\left(H^{0}\left(Y, \mathcal{K}_{Y}^{*}\right)\right)$ ce qui montre que $D=0$ dans $\mathrm{CaCl}_{G}(X)$.

La surjectivité est la partie difficile. Soit $\mathcal{L}$ un $G$-faisceau inversible sur $X$, on veut montrer qu'il peut-être représenté par un diviseur $G$-invariant sur $X$. Pour cela on note qu'il suffit de montrer :

$$
\text { Il existe une injection équivariante }: 0 \rightarrow \mathcal{L} \rightarrow \mathcal{K}_{X}
$$

Supposons en effet cette condition réalisée, alors [14] II 6.13 montre qu'il existe un unique diviseur de cartier $D$ sur $X$ tel qu'on ait un isomorphisme (à priori non équivariant) de faisceaux plongés $\mathcal{L} \simeq \mathcal{L}(D)$, ce dont on déduit des isomorphismes (à priori non équivariants) de faisceaux plongés

$$
\mathcal{L}(g D) \simeq g_{*}(\mathcal{L}(D)) \simeq g_{*}(\mathcal{L}) \simeq \mathcal{L} \simeq \mathcal{L}(D)
$$

ce qui montre que $D$ est $G$-invariant et que $\mathcal{L} \simeq \mathcal{L}(D)$ comme $G$-faisceau.

Reste à montrer l'assertion ci-dessus. Comme on a une injection naturelle $\mathcal{L} \rightarrow \mathcal{K}_{X} \otimes_{\mathcal{O}_{X}} \mathcal{L}$ il suffit de montrer que $\mathcal{K}_{X} \otimes_{\mathcal{O}_{X}} \mathcal{L} \simeq \mathcal{K}_{X}$. D'après [14] II 6.15 ces deux faisceaux sont constants (comme faisceaux de modules sur $X$ ). On va comparer leurs fibres au point générique. Notons $K(X)$ le corps des fractions de $X$ et $\xi$ son point générique. En tirant $\mathcal{L}$ par le $G$-morphisme $\xi \rightarrow X$ on voit que $\mathcal{L}_{\xi}$ est un $G$-faisceau inversible sur $\xi$, autrement dit c'est un $K(X) \circ G$-module de dimension 1 comme $K(X)$-espace vectoriel. Or on a vu au 2.1.3 que les $K(X) \circ G$ modules sont classifiés par leur rang sur $K(X)$, ce qui donne $\left(\mathcal{K}_{X} \otimes_{\mathcal{O}_{X}} \mathcal{L}\right)_{\xi} \simeq \mathcal{K}_{X, \xi}$. Pour conclure on applique le lemme suivant.

\section{Lemme 2.38}

Soient $X$ un schéma intègre de point générique $\xi, \mathcal{F}$ et $\mathcal{G}$ deux $G$-faisceaux constants comme faisceaux de modules sur $X$. On suppose $\mathcal{F}_{\xi} \simeq \mathcal{G}_{\xi}$ comme $K(X) \circ G$ modules. Alors $\mathcal{F} \simeq \mathcal{G}$ comme $G$-faisceaux.

\section{Démonstration :}

On peut traduire les hypothèses sur $\mathcal{F}$ et $\mathcal{G}$ en disant qu'on s'est donné deux $K(X) \circ G$-modules $A$ et $B$, ce qui permet de voir $\mathcal{F}$ comme faisceau des fonctions localement constantes de $X$ dans $A$, muni de la $G$-linéarisation :

$$
\begin{aligned}
& \mathcal{F}\left(g^{-1} U\right) \longrightarrow \mathcal{F}(U) \\
& f \longrightarrow g \circ f \circ g^{-1}
\end{aligned}
$$


et de même pour $B$. L'hypothèse au point générique fait que l'on dispose d'un $G$-isomorphisme $\phi: A \simeq B$ qui permet de construire un $G$-isomorphisme entre $\mathcal{F}$ et $\mathcal{G}$ donné par :

$$
\begin{aligned}
\mathcal{F}(U) \longrightarrow \mathcal{G}(U) & \longrightarrow \phi \circ f
\end{aligned}
$$

\section{Isomorphisme entre $\operatorname{Pic}_{G}(X)$ et $H^{1}\left(X, G, \mathcal{O}_{X}^{*}\right)$}

Les deux sections précédentes suffisent évidemment à démontrer le théorème 2.34. Cependant le cas classique et l'emploi du lemme de Dedekind pour démontrer les deux isomorphismes précédents (camouflé dans le théorème 90 de Hilbert et la classification des $K(X) \circ G$-modules) montrent l'intêret de décrire directement le lien entre $\operatorname{Pic}_{G}(X)$ et $H^{1}\left(X, G, \mathcal{O}_{X}^{*}\right)$. Un avantage supplémentaire de cette approche est de montrer que ces deux groupes sont isomorphes pour tout $G$-schéma $X$, et en particulier lorsque l'action n'est pas fidèle. Comme ce résultat ne sera pas nécessaire par la suite, on ne fait qu'ébaucher la description de cet isomorphisme.

Pour décrire ce lien, on va montrer, en suivant l'exposé fait dans [22], comment associer un certain 1-cocycle à toute classe d'isomorphisme de $G$-faisceaux inversibles sur $X$. On commence par décrire le groupe de cohomologie de Cech $\check{H}^{1}(\mathcal{U}, G, \mathcal{F})$ associé à un recouvrement ouvert $\mathcal{U}=\left(U_{i}\right)_{i \in I}$ et à un $G$-faisceau abélien $\mathcal{F}$. Pour simplifier, on supposera les ouverts du recouvrement $G$-stables. Le groupe des $q$-chaînes de Cěch $C^{q}(\mathcal{U}, \mathcal{F})$ est alors muni d'une $G$-action naturelle, on peut donc considérer sa résolution standart en tant que $G$-module, et on note alors $C^{p, q}=C^{p}\left(G, C^{q}(\mathcal{U}, \mathcal{F})\right)$ le complexe double obtenu. Par définition $\check{H}^{n}(\mathcal{U}, G, \mathcal{F})$ désigne la cohomologie du complexe total $T^{n}$ associé. Pour décrire $\check{H}^{1}(\mathcal{U}, G, \mathcal{F})$ on considère le diagramme suivant :

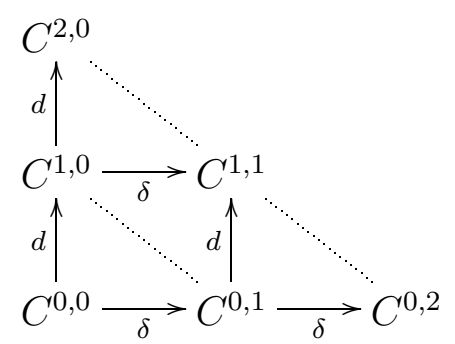

$\delta$ désigne la différentielle de la cohomologie de Cěch, $d$ celle de la cohomologie des groupes, plus précisement :

$$
\begin{gathered}
C^{1,0} \longrightarrow C^{1,1} \\
f=\left(f_{i}\right)_{i \in I} \longrightarrow\left(g \rightarrow\left(f_{j}(g)-f_{i}(g)\right)_{i, j \in I}\right)
\end{gathered}
$$




$$
\begin{gathered}
C^{0,1} \longrightarrow C^{1,1} \\
\left(f_{i, j}\right)_{i, j \in I} \longrightarrow\left(g \rightarrow\left(f_{i, j}^{g}-f_{i, j}\right)_{i, j \in I}\right)
\end{gathered}
$$

Par définition une 1-cochaîne de ce complexe total est un élément $\left(\left(f_{i}\right)_{i \in I},\left(f_{i, j}\right)_{i, j \in I}\right)$ de $T^{1}=C^{1,0} \oplus C^{0,1}$. Comme la composante de la différentielle $T^{1} \rightarrow T^{2}$ sur $C^{1,1}$ s'écrit $d-\delta$, cette 1-cochaîne est un 1-cocycle si $f=\left(f_{i}\right)_{i \in I}$ est un 1-cocycle pour la cohomologie des groupes, $\left(f_{i, j}\right)_{i, j \in I}$ est un 1-cocycle pour la cohomologie de Cěch, et si de plus :

$$
\forall g \in G \quad \forall i, j \in I \quad f_{j}(g)-f_{i}(g)=f_{i, j}^{g}-f_{i, j}
$$

Les 1-cobords dans $T^{1}$ sont décrits de la manière évidente.

Soit à présent un $G$-faisceau inversible $\mathcal{L}$. Soit $\mathcal{U}=\left(U_{i}\right)_{i \in I}$ un recouvrement ouvert tel que pour tout $i$ on ait $\mathcal{L}_{\mid U_{i}} \simeq \mathcal{O}_{U_{i}}$, pour simplifier, on supposera à nouveau les $U_{i} G$-stables. Pour chaque $i$ dans $I$, on choisit un générateur $m_{i}$ de $\mathcal{L}\left(U_{i}\right)$ sur $\mathcal{O}_{X}\left(U_{i}\right)$. Ce choix détermine, pour chaque couple $i, j$ d'indices de $I$, un unique $f_{i, j}$ dans $\mathcal{O}_{X}^{*}\left(U_{i} \cap U_{j}\right)$ tel que $m_{j}=f_{i, j} m_{i}$ sur $\mathcal{L}\left(U_{i} \cap U_{j}\right)$, et pour chaque indice $i$ de $I$ et chaque élément $g$ de $G$, un unique élément $f_{i}(g)$ de $\mathcal{O}_{X}^{*}\left(U_{i}\right)$ tel que $m_{i}^{g}=f_{i}(g) m_{i}$.

\section{Lemme 2.39}

Le couple $\left(\left(f_{i}\right)_{i \in I},\left(f_{i, j}\right)_{i, j \in I}\right)$ détermine un 1-cocycle dont la classe dans $\check{H}^{1}\left(\mathcal{U}, G, \mathcal{O}_{X}^{*}\right)$ est indépendante du choix des $m_{i}$.

\section{Démonstration :}

Immédiat, la relation de cocycle ci-dessus se déduisant du fait que $\mathcal{L}$ est un $G$ module.

Il reste à montrer qu'on peut choisir le recouvrement $\mathcal{U}$ de manière à ce que le morphisme $\operatorname{Pic}_{G} X \rightarrow H^{1}\left(\mathcal{U}, G, \mathcal{O}_{X}^{*}\right)$ défini par le lemme ci-dessus soit un isomorphisme, et également de manière à ce que les groupes $H^{1}\left(\mathcal{U}, G, \mathcal{O}_{X}^{*}\right)$ et $H^{1}\left(X, G, \mathcal{O}_{X}^{*}\right)$ coïncident.

\subsubsection{Application à l'étude de $\operatorname{Pic}_{G}(X)$}

\section{Première suite exacte fondamentale}

La suite exacte à cinq termes associée à la première suite spectrale fondamentale donne :

$0 \rightarrow H^{1}\left(G, \Gamma_{X}\left(\mathcal{O}_{X}\right)^{*}\right) \rightarrow \operatorname{Pic}_{G} X \rightarrow(\operatorname{Pic} X)^{G} \rightarrow H^{2}\left(G, \Gamma_{X}\left(\mathcal{O}_{X}\right)^{*}\right) \rightarrow H^{2}\left(X, G, \mathcal{O}_{X}^{*}\right)$

Comme on va le voir, cette suite exacte permet de discuter l'existence d'une 
$G$-linéarisation sur un faisceau inversible donné, et permet aussi de classifier les différentes $G$-linéarisations dont on peut équiper un même faisceau.

Plus précisément l'exactitude en $\operatorname{Pic}_{G} X$ signifie que si $\mathcal{L}$ est un $G$-faisceau inversible, les différentes $G$-linéarisations dont on peut équiper le faisceau sousjacent, modulo les $G$-linéarisations induites par les isomorphismes du faisceau structurel, sont en bijection avec $H^{1}\left(G, \Gamma_{X}\left(\mathcal{O}_{X}\right)^{*}\right)$. On va redémontrer ce fait de manière élémentaire. Clairement, il suffit de prouver l'affirmation suivante :

\section{Lemme 2.40}

Les $G$-linéarisations de $\mathcal{O}_{X}$ sont en bijection avec $Z^{1}\left(G, \Gamma_{X}\left(\mathcal{O}_{X}\right)^{*}\right)$

\section{Démonstration :}

On rappelle qu'on note $g^{\#}: \mathcal{O}_{X} \rightarrow g_{*} \mathcal{O}_{X}$ l'isomorphisme de faisceaux d'anneaux sur $X$ correspondant à l'automorphisme de schémas $g: X \rightarrow X$. Les $\left(g^{\#}\right)^{-1}$ constituent la $G$-linéarisation naturelle de $\mathcal{O}_{X}$ et induisent une action sur l'anneau $\Gamma_{X}\left(\mathcal{O}_{X}\right)$. On peut identifier $\Gamma_{X}\left(\mathcal{O}_{X}\right)^{*}$ à $\operatorname{Aut}\left(\mathcal{O}_{X}\right)$ muni de l'action $\psi \rightarrow\left(g^{\#}\right)^{-1} \circ$ $g_{*} \psi \circ g^{\#}$ comme groupes abéliens munis d'une action de $G$. On identifiera donc $Z^{1}\left(G, \Gamma_{X}\left(\mathcal{O}_{X}\right)^{*}\right)$ à $Z^{1}\left(G, \operatorname{Aut}\left(\mathcal{O}_{X}\right)\right)$ par la suite.

On fixe une $G$-linéarisation $\left(\psi_{g}\right)_{g \in G}$ de $\mathcal{O}_{X}$. On définit alors un cocycle de $Z^{1}\left(G, \Gamma_{X}\left(\mathcal{O}_{X}\right)^{*}\right)$ par :

$$
\begin{aligned}
& G \longrightarrow \operatorname{Aut}\left(\mathcal{O}_{X}\right) \\
& g \longrightarrow \psi_{g} \circ g^{\#}
\end{aligned}
$$

Réciproquement, tout cocycle dans $Z^{1}\left(G, \Gamma_{X}\left(\mathcal{O}_{X}\right)^{*}\right)$ détermine une $G$-linéarisation de $\mathcal{O}_{X}$, d'où la bijection annoncée.

\section{Remarque :}

Soit $\psi$ dans $\operatorname{Aut}\left(\mathcal{O}_{X}\right)$. Le cobord $g \rightarrow \psi^{-1} \circ \psi^{g}$ correspond par la bijection cidessus au pull-back de la $G$-linéarisation naturelle sur $\mathcal{O}_{X}$ par $\psi$.

On considère à présent l'exactitude en $(\operatorname{Pic} X)^{G}$. Soit $\mathcal{L}$ un $G$-faisceau inversible sur $X$. Pour que $\mathcal{L}$ admette une $G$-linéarisation il est bien sur nécessaire que pour tout $g$ dans $G$ on ait un isomorphisme $g_{*} \mathcal{L} \simeq \mathcal{L}$, mais ce n'est pas toujours suffisant. La suite exacte ci-dessus situe l'obstruction dans $H^{2}\left(G, \Gamma_{X}\left(\mathcal{O}_{X}\right)^{*}\right)$, ce qu'on précise dans le lemme suivant. On utilise l'isomorphisme naturel $\operatorname{Aut}(\mathcal{L}) \simeq$ $\Gamma_{X}\left(\mathcal{O}_{X}\right)^{*}$.

\section{Lemme 2.41}

Soit $\mathcal{L}$ un $G$-faisceau inversible sur $X$, tel que pour tout $g$ dans $G g_{*} \mathcal{L} \simeq \mathcal{L}$. On choisit un tel isomorphisme $\psi_{g}$ pour tout $g$ dans $G$. Alors l'application :

$$
\begin{aligned}
& G \times G \longrightarrow \Gamma_{X}\left(\mathcal{O}_{X}\right)^{*} \\
&(h, g) \longrightarrow \psi_{h} \circ h_{*} \psi_{g} \circ \psi_{h g}^{-1}
\end{aligned}
$$


est un 2-cocycle. De plus sa classe dans $H^{2}\left(G, \Gamma_{X}\left(\mathcal{O}_{X}\right)^{*}\right)$ est indépendante $d u$ choix des $\psi_{g}$ et s'annule si et seulement si $\mathcal{L}$ peut être équipé d'une $G$-linéarisation.

\section{Démonstration :}

On note d'abord que si $\mathcal{M}$ et $\mathcal{N}$ sont deux faisceaux inversibles plongés dans $\mathcal{K}$, alors $\operatorname{Hom}(\mathcal{M}, \mathcal{N})=H^{0}\left(X, \mathcal{M} \otimes_{\mathcal{O}_{X}} \check{\mathcal{N}}\right)$ est naturellement inclus dans le corps des fonctions $K(X)$. De plus cette inclusion transforme la composition en produit et est compatible avec l'action. On en déduit que l'application composée :

$$
G \times G \rightarrow \Gamma_{X}\left(\mathcal{O}_{X}\right)^{*} \rightarrow K(X)^{*}
$$

définit un 2-cobord. En considérant l'injection entre complexe de chaînes :

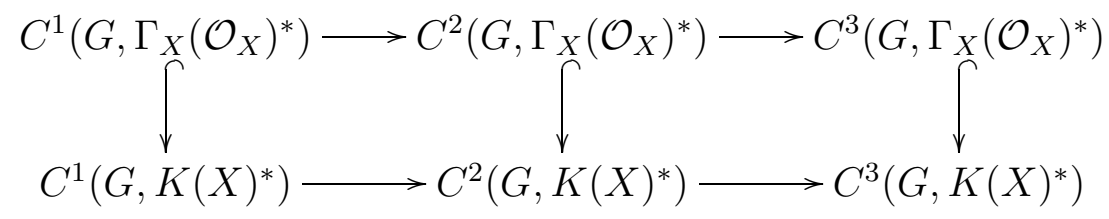

on en déduit le fait que $G \times G \rightarrow \Gamma_{X}\left(\mathcal{O}_{X}\right)^{*}$ est un 2-cocycle. Les autres affirmations du lemme ne posent pas de problème.

\section{Deuxième suite exacte fondamentale}

Dans ce paragraphe, on va examiner le problème suivant :

Problème 2.42

Soit $\mathcal{L}$ un $G$-faisceau inversible sur $X$.

A quelle condition existe-t-il un faisceau $\mathcal{M}$ sur $Y$ tel que $\mathcal{L}=\pi^{*} \mathcal{M}$ ?

Pour répondre à ce problème, un outil important est la deuxième suite exacte fondamentale associée au faisceau $\mathcal{O}_{X}^{*}$ qui s'écrit :

$0 \rightarrow \operatorname{Pic} Y \rightarrow \operatorname{Pic}_{G} X \rightarrow \Gamma_{Y}\left(R^{1} \pi_{*}^{G} \mathcal{O}_{X}^{*}\right) \rightarrow H^{2}\left(Y, \pi_{*}^{G} \mathcal{O}_{X}^{*}\right) \rightarrow H^{2}\left(X, G, \mathcal{O}_{X}^{*}\right)$

L'exactitude en $\operatorname{Pic}_{G} X$ montre en particulier que l'obstruction à donner une réponse positive au problème 2.42 est mesurée par une classe de cohomologie dans $\Gamma_{Y}\left(R^{1} \pi_{*}^{G} \mathcal{O}_{X}^{*}\right)$. Comme ce groupe semble plutôt difficile à calculer en général, on va en donner une interprétation géomètrique locale.

Soit donc $P$ un point fermé de $X, G_{P}$ le groupe de décomposition de $P, X^{\prime}$ la $G$-sous-variété de dimension zéro $X^{\prime}=G \times{ }^{G_{P}} X, Q=\pi(P)$, $Y^{\prime}$ le quotient $X^{\prime} / G$, qui s'identifie au point $Q=\pi(P)$ de $Y$. Les notations des quotients et des immersions fermées intervenant sont fixées par le diagramme commutatif suivant: 


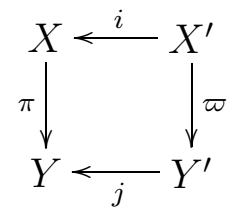

Ce diagramme permet de définir facilement une obstruction locale à une réponse positive au problème 2.42. En effet il induit un diagramme commutatif :

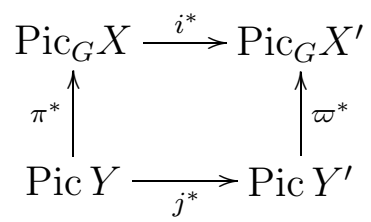

On a $\operatorname{Pic} Y^{\prime}=0$ donc si un $G$-faisceau inversible $\mathcal{L}$ sur $X$ est dans l'image de $\pi^{*}$, il vérifie nécessairement $i^{*} \mathcal{L}=0$. Cette condition locale a l'avantage d'avoir une interprétation géomètrique simple, par exemple si $X$ est un schéma sur un corps algébriquement clos $k$, sur lequel $G$ agit par $k$-automorphismes, le groupe $\operatorname{Pic}_{G} X^{\prime}$ s'identifie à $\operatorname{Hom}\left(G_{P}, k^{*}\right)$. Autrement dit, le $G$-faisceau $\mathcal{L}$ détermine alors un caractère de $G_{P}$, qui doit s'annuler si le problème 2.42 admet une réponse positive pour $\mathcal{L}$. (Le cas d'un $G$-schéma général est à peine plus compliqué, puisque $\operatorname{Pic}_{G} X^{\prime}$ s'identifie à $H^{1}\left(G_{P}, k(P)^{*}\right)$ où $k(P)$ est le corps résiduel en $P$ ). L'inconvénient de ce type de condition locale est qu'il s'agit évidemment d'une condition nécessaire, mais pas suffisante pour pouvoir donner une réponse positive au problème 2.42 .

Par la suite, on se propose de montrer comment, de manière imagée, les obstructions locales déterminent les obstructions locales. Plus précisement on va montrer l'existence d'un morphisme naturel

$$
\Gamma_{Y}\left(R^{1} \pi_{*}^{G} \mathcal{O}_{X}^{*}\right) \rightarrow \operatorname{Pic}_{G} X^{\prime}
$$

factorisant le morphisme $i^{*}$ du diagramme ci-dessus. Pour cela on remarque que le morphisme de faisceaux d'anneaux canonique $\mathcal{O}_{X} \rightarrow i_{*} \mathcal{O}_{X^{\prime}}$ induit un morphisme de faisceaux de groupes abéliens $R^{1} \pi_{*}^{G} \mathcal{O}_{X}^{*} \rightarrow R^{1} \pi_{*}^{G}\left(i_{*} \mathcal{O}_{X^{\prime}}^{*}\right)$. D'autre part, d'après le lemme ci-dessous, le faisceau $R^{1} \pi_{*}^{G}\left(i_{*} \mathcal{O}_{X^{\prime}}^{*}\right)$ s'identifie à $j_{*}\left(R^{1} \varpi_{*}^{G} \mathcal{O}_{X^{\prime}}^{*}\right)$. Enfin, d'après la deuxième suite exacte fondamentale sur $X^{\prime}$, appliquée au faisceau $\mathcal{O}_{X^{\prime}}^{*}$, le groupe $\Gamma_{Y^{\prime}}\left(R^{1} \varpi_{*}^{G} \mathcal{O}_{X^{\prime}}^{*}\right)$ s'identifie à $\mathrm{Pic}_{G} X^{\prime}$. Il reste donc à démontrer le lemme suivant :

\section{Lemme 2.43}

Les foncteurs $R^{1} \pi_{*}^{G} \circ i_{*}$ et $j_{*} \circ R^{1} \varpi_{*}^{G}$ sont isomorphes. 


\section{Démonstration :}

Le diagramme commutatif de foncteurs

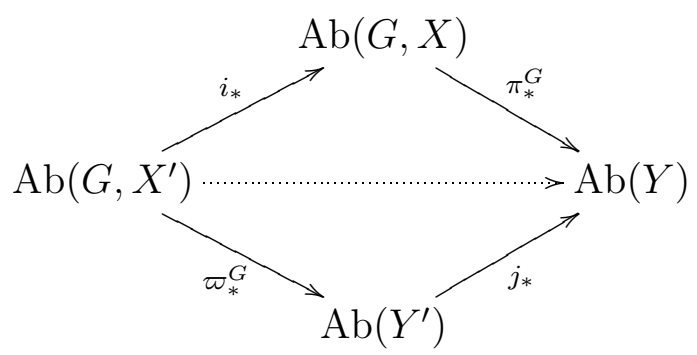

permet de décomposer le foncteur correspondant à la ligne horizontale de deux manières. Le lemme ne fait qu'exprimer le premier foncteur dérivé de ce foncteur suivant ces deux décompositions, compte-tenu du théorème A.4 et du fait que les foncteurs $i_{*}$ et $j_{*}$ sont exacts. 


\section{Chapitre 3}

\section{Formule de Riemann-Roch équivariante}

\subsection{Introduction}

L'idée relativement courante de construire une théorie équivariante des courbes a une justification de nature fonctorielle, qui peut être résumée ainsi. Si $X$ est une courbe projective sur un corps algébriquement clos $k$, de nombreuses formules concernant des invariants entiers relatifs à $X$ sont en fait des égalités entre dimensions de certains $k$-espaces vectoriels, et proviennent d'isomorphismes entre ces espaces. Si de plus la courbe $X$ est munie d'une action d'un groupe fini $G$, on peut espèrer que ces isomorphismes commutent avec l'action de $G$, et qu'ils fournissent ainsi des égalités entre les caractères associés aux représentations de $G$ correspondantes. On est ainsi amené à rechercher des formules non plus à valeur dans $\mathbb{Z}$, mais dans l'anneau des caractères du groupe $R_{k}(G)$.

Dans ce chapitre, on va s'intéresser aux analogues équivariants du théorème classique de Riemann-Roch sur les courbes. De telles formules ont été établies au début des années 1980 tout d'abord par Ellingsrud et Lønsted ([7]) dans le cas d'une action réductive, puis par Nakajima ([24]) dans le cas d'une action modérée. Bien que d'un intérêt pratique considérable, ces résultats comportent cependant certaines limites qui en restreignent l'usage, en particulier leur mauvais comportement fonctoriel. Autrement dit, ces formules ne se spécialisent pas "manifestement" en les formules classiques lorsque on les applique au cas de l'action triviale.

Le résultat principal de ce chapitre (théorème 3.11) est une formule de RiemannRoch équivariante présentant deux avantages. D'une part, elle s'envoie sur la formule classique par le morphisme $\operatorname{dim}: R_{k}(G) \rightarrow \mathbb{Z}$. D'autre part, elle est valable sans hypothèse sur la nature de l'action, y compris dans le cas d'une action sauvage. Pour permettre à cette formule d'être fonctorielle, on a dû introduire à la section 3.4 la notion de degré équivariant d'un $G$-faisceau inversible, ce qui est 
rendu possible par notre étude du groupe de Picard équivariant d'un $G$-schéma intègre au chapitre précédent (théorème 2.34). Ce degré équivariant s'exprime en termes de caractères de ramification de l'action, qui sont des caractères des stabilisateurs des points fixes, qu'on définit précisement dans la section 3.3.

Dans les deux sections suivantes, on donne des applications du théorème 3.11. Ce théorème permet de donner des preuves concises de résultats déja connus, l'exemple le plus frappant étant le lemme 3.18, qui peut être aussi déduit de [25], Theorem 1. Dans la section 3.6, on s'intéresse à la structure galoisienne de l'espace des sections globales des $G$-faisceaux inversibles de grand degré, qu'on explicite complètement dans le cas d'une action modérée (théorème 3.22), retrouvant ainsi un résultat de Nakajima. Pour cela, on établit auparavant dans ce cadre une formule de Hurwitz équivariante (théorème 3.16). Dans la section 3.7, on aborde le problème de la structure galoisienne de l'espace des différentielles holomorphes sur la courbe considérée. En se plaçant d'abord dans le cas modéré, on donne une preuve directe d'un résultat de Kani (théorème 3.26) concernant la structure d'un espace de différentielles logarithmiques le long du lieu de ramification de l'action. Dans le cas sauvage, on prouve une formule "à la Weil" (théorème 3.28). Outre le théorème 3.11, un ingrédient essentiel est le théorème 3.23 qui relie les représentations d'Artin de $\pi: X \rightarrow Y=X / G$ au degré équivariant du faisceau des différentielles, et qui semble d'un intérêt indépendant.

\subsection{Notations}

\subsubsection{Définitions}

Le mot courbe désignera une courbe projective et lisse sur un corps algébriquement clos $k$ de caractéristique $p$ (au sens de [14], Chapter IV). Elle sera toujours supposée irréductible, et on notera $\xi$ son point générique. On parlera des points d'une courbe pour désigner ses points fermés. Si $X$ est une courbe on notera $\operatorname{Aut}_{k}(X)$ le groupe de ses automorphismes fixant le corps de base $k$. Les actions de groupe sur les courbes considérées seront fidèles et définies sur $k$, autrement dit si $X$ est une courbe et $G$ est un groupe, une action de $G$ sur $X$ est la donnée d'un morphisme injectif $G \rightarrow \operatorname{Aut}_{k}(X)$. On supposera toujours le groupe $G$ fini. Cette condition n'est pas trop limitative : en effet il est connu que les courbes de genre supérieur ou égal à 2 ont un groupe d'automorphismes fini. Les faits suivants sont également bien connus.

Fait 3.1

Si $X$ est une courbe munie d'une action d'un groupe fini $G$, alors $X$ est un $G$-schéma admissible.

En effet toute orbite sous $G$ est contenue dans un ouvert affine (on peut prendre le complémentaire de n'importe quel point n'appartenant pas à cette orbite, par 
exemple).

\section{Fait 3.2}

Si $X$ est une courbe munie d'une action d'un groupe fini $G$, de quotient $\pi: X \rightarrow$ $Y=X / G$, alors $Y$ est lisse.

En effet, $X$ étant lisse et de dimension 1, $X$ est en fait normale, ce dont on déduit sans difficulté que $Y$ est normale, donc lisse.

\subsubsection{Ramification de l'action}

Soit $P$ un point de $X$, on note $G_{P}=\{g \in G / g P=P\}$ son stabilisateur, et $e_{P}$ le cardinal de $G_{P}$. D'un point de vue arithmétique, $G_{P}$ est aussi le groupe de décomposition en $P$ de l'extension $\pi: X \rightarrow Y=X / G$; il coïncide avec le groupe d'inertie en $P$, puisque le corps de base est supposé algébriquement clos. On notera $X_{\text {ram }}$ l'ensemble fini des points de $X$ à inertie non triviale.

\section{Définition 3.3}

L'action de $G$ sur $X$ est dite modérée si l'ordre des stabilisateurs est premier à la caractéristique du corps de base ; elle est dite libre si tous les stabilisateurs sont triviaux.

Dans le cas d'une action modérée sur une courbe, on peut préciser la structure des stabilisateurs :

\section{Fait 3.4}

Si l'action de $G$ sur $X$ est modérée, les stabilisateurs sont cycliques.

Ceci découle par exemple de [30], Chapitre IV, §2, Corollaire 1.

\subsection{Caractères de ramification}

\subsubsection{Définition}

Par la suite on notera $\mathcal{O}_{X, P}$ l'anneau local d'un point $P$ de $X$ et $\mathfrak{m}_{P}$ son idéal maximal. On appellera espace cotangent $a ̀ X$ en $P$ le $k$-espace vectoriel unidimensionel $\mathfrak{m}_{P} / \mathfrak{m}_{P}^{2}$. Enfin pour tout groupe $G, \hat{G}$ désignera l'ensemble des caractères irréductibles de $G$, c'est un groupe égal à $\operatorname{Hom}\left(G, k^{*}\right)$ si $G$ est commutatif.

\section{Définition 3.5}

Soit $P$ un point de $X$. On appelle caractère de ramification de $X$ en $P$ et on note $\psi_{P}$ le caractère de $G_{P}$ par lequel $G_{P}$ agit sur l'espace cotangent à $X$ en $P$.

\section{Proposition 3.6}

(i) $\forall f \in k(X)^{*} \forall g \in G_{P} \quad f^{g} / f \equiv \psi_{P}(g)^{v_{P}(f)} \bmod \mathfrak{m}_{P}$ dans $\mathcal{O}_{X, P}$ 
(ii) $\forall \omega \in \Omega_{X, \xi}^{*} \forall g \in G_{P} \quad \omega^{g} / \omega \equiv \psi_{P}(g)^{v_{P}(\omega)+1} \bmod \mathfrak{m}_{P}$ dans $\mathcal{O}_{X, P}$

(iii) Si $P^{\prime}=g P$ alors $\psi_{P^{\prime}}=\psi_{P}^{g}$ (i.e. $\forall g^{\prime} \in G_{P^{\prime}} \psi_{P^{\prime}}\left(g^{\prime}\right)=\psi_{P}\left(g^{-1} g^{\prime} g\right)$ )

(iv) Si la ramification est modérée $\psi_{P}$ est un générateur de $\hat{G}_{P}$.

\section{Démonstration :}

Immédiat, sauf pour le (iv), qui découle de [30], Chapitre IV, §2, Corollaire 1.

\subsubsection{Cas d'une action réductive d'un groupe cyclique à ramification totale}

A titre d'exemple, on considère le cas d'une courbe $X$ munie d'une action d'un groupe fini $G \simeq \mathbb{Z} /(m)$ avec $m$ premier à $p$, telle que la ramification soit totale. C'est un cadre d'étude agréable, et beaucoup de problèmes du cas général s'y posent de manière non triviale, certains s'y ramenant même directement.

La proposition suivante montre qu'il existe dans ce cas une relation globale entre les caractères de ramification :

\section{Proposition 3.7}

Soit $\chi$ un caractère de dimension 1 de $G$. Pour tout $P$ dans $X_{\text {ram }}$, $\chi$ s'écrit $\chi=\psi_{P}^{a_{\chi, P}}$ pour des entiers $a_{\chi, P}$. On a la relation :

$$
\sum_{P \in X_{\text {ram }}} a_{\chi, P} \equiv 0 \bmod m
$$

\section{Remarque :}

Ce fait bien connu nous a été signalé par Michel Matignon. Faute de références, nous en donnons une preuve. Les entiers $a_{\chi, P}$ sont parfois appelés données d'Hurwitz.

\section{Démonstration :}

On utilise la théorie de Kummer. Plus précisement on a une suite exacte courte de $G$-modules :

$$
0 \rightarrow \mu_{m} \rightarrow k(X)^{*} \rightarrow\left(k(X)^{*}\right)^{m} \rightarrow 0
$$

La suite exacte longue de cohomologie galoisienne associée et le théorème 90 de Hilbert montrent que l'homomorphisme de connexion $\delta:\left[\left(k(X)^{*}\right)^{m}\right]^{G} \rightarrow \hat{G}$ est surjectif, et donc qu'il existe une fonction $f_{\chi}$ dans $k(X)^{*}$ telle que :

$$
\forall g \in G \quad f_{\chi}^{g}=\chi(g) f_{\chi}
$$

La proposition 3.6 (i) montre alors qu'on a pour tout $P$ de $X_{\text {ram }}$ :

$a_{\chi, P} \equiv v_{P}\left(f_{\chi}\right) \bmod m$. La proposition se déduit alors des deux faits suivants : $\operatorname{deg}\left(\left(f_{\chi}\right)\right)=0$ d'une part, et d'autre part comme le diviseur $\left(f_{\chi}\right)$ est $G$-invariant :

$$
\operatorname{deg}\left(\left(f_{\chi}\right)\right) \equiv \sum_{P \in X_{\text {ram }}} v_{P}\left(f_{\chi}\right) \quad \bmod m
$$




\subsection{Degré équivariant}

\subsubsection{Degré équivariant d'un diviseur invariant}

On appelera orbite réduite tout diviseur du type $D^{\prime}=\sum_{\sigma \in G \backslash G_{P}} P^{\sigma}$ où $P$ est un point de $X, G_{P}$ est son stabilisateur dans $G$, et $G \backslash G_{P}$ l'ensemble des classes à gauche de $G$ modulo $G_{P}$. On appelera de même orbite tout diviseur du type $D=r D^{\prime}$ où $r \in \mathbb{Z}$ et où $D^{\prime}$ est une orbite réduite. Les orbites engendrent $(\operatorname{Div} X)^{G}$ comme groupe ce qui permet de poser la définition suivante :

\section{Définition 3.8}

On définit l'application degré équivariant $\operatorname{deg}_{e q}:(\operatorname{Div} X)^{G} \rightarrow R_{k}(G)$ comme étant l'application vérifiant les propriétés suivantes :

1. $\operatorname{deg}_{\text {eq }}$ est additif sur les G-diviseurs à supports disjoints ;

2. soit $D=r D^{\prime}$ une orbite comme ci-dessus. On pose :

$$
\operatorname{deg}_{e q}(D)=\left\{\begin{array}{cc}
\operatorname{Ind}_{G_{P}}^{G} \sum_{l=1}^{r} \psi_{P}^{-l} & \text { si } r>0 \\
0 & \text { si } r=0 \\
-\operatorname{Ind}_{G_{P}}^{G} \sum_{l=0}^{-(r+1)} \psi_{P}^{l} & \text { si } r<0
\end{array}\right.
$$

\section{Remarques :}

1. Le fait que dans la formule ci-dessus $\operatorname{deg}_{e q}(D)$ est bien défini indépendemment du choix de $P$ résulte du lemme 3.6, (iii).

2. Le degré équivariant n'a pas de bonnes propriétés fonctorielles : il n'est pas additif, de plus il n'est pas clair que l'on ait un analogue équivariant de la formule classique $\operatorname{deg}\left(f^{*}(D)\right)=\operatorname{deg}(f) \cdot \operatorname{deg}(D)$.

\subsubsection{Degré équivariant d'un $G$-faisceau inversible}

D'après le théorème 2.34 on a un isomorphisme entre $\operatorname{Pic}_{G} X$ et $\mathrm{CaCl}_{G} X$. Comme la courbe $X$ étudiée est lisse le groupe des diviseurs de Cartier sur $X$ coïncide avec le groupe des diviseurs de Weil sur $X$ ([14] Chapter II Proposition 6.11) et on a donc un isomorphisme :

$$
\operatorname{Pic}_{G} X \simeq(\operatorname{Div} X)^{G} / \pi^{*}(\operatorname{Princ}(Y))
$$

où Princ $(Y)$ ) désigne le groupe des diviseurs principaux sur $Y$.

Le corollaire de la proposition suivante montre que l'application $\operatorname{deg}_{e q}$ définie au paragraphe précédent est invariante sur les classes modulo $\pi^{*}(\operatorname{Princ}(Y))$, et induit donc une application $\operatorname{deg}_{e q}: \operatorname{Pic}_{G} X \longrightarrow R_{k}(G)$. 
Proposition 3.9

Soit $H$ un groupe fini produit semi-direct d'un sous-groupe cyclique $Z$ d'ordre $e_{0}$ premier à $p$ et d'un sous-groupe distingué $P$ d'ordre une puissance de $p$. Soit de plus $\psi^{\prime}$ un caractère primitif de $Z$ et $\psi$ le caractère de $H$ correspondant. Alors on a l'égalité suivante dans $R_{k}(H)$ :

$$
\sum_{l=1}^{\# H} \psi^{l}=[k[H]]
$$

Démonstration :

D'après [31], §18, Corollaire 1 du Théorème 42, il suffit de montrer l'égalité des caractères de Brauer des représentations $x=\sum_{l=1}^{\# H} \psi^{l}$ et $y=[k[H]]$, qu'on note respectivement $\phi_{x}$ et $\phi_{y}$. Soit $h$ un élément $p$-régulier non trivial de $H$. Comme $\psi^{\prime}$ est supposé primitif, $\psi(h)$ est une racine $e_{0}$-ième de l'unité non triviale. On en déduit que le caractère $\phi_{x}$ est donné par :

$$
\phi_{x}(h)=\left\{\begin{array}{ccc}
\# H & \text { si } & h=1 \\
0 & \text { si } & h \neq 1
\end{array}\right.
$$

et donc $\phi_{x}=\phi_{y}$, d'où le lemme.

Corollaire 3.10

Soient $D$ un diviseur $G$-invariant sur $X$ et $\delta$ est un diviseur sur $Y$. On a :

$$
\operatorname{deg}_{e q}\left(D+\pi^{*}(\delta)\right)=\operatorname{deg}_{e q} D+\operatorname{deg} \delta[k[G]]
$$

\section{Démonstration :}

On peut supposer que le support de $\delta$ est réduit à un point $Q$. Soit $P$ un antécédent de $Q$ par $\pi: X \rightarrow Y$. D'après [30], $\S I V$, Corollaire 4 de la Proposition 7 , on peut appliquer le lemme ci-dessus au stabilisateur $G_{P}$ de $P$, ce qui donne l'égalité annoncée.

\section{Remarque :}

Le corollaire montre que l'information contenue dans le degré équivariant modulo $([k[G]])=\{m[k[G]], m \in \mathbb{Z}\}$ est concentrée aux points d'inertie non nulle. Plus précisement le diagramme suivant commute :

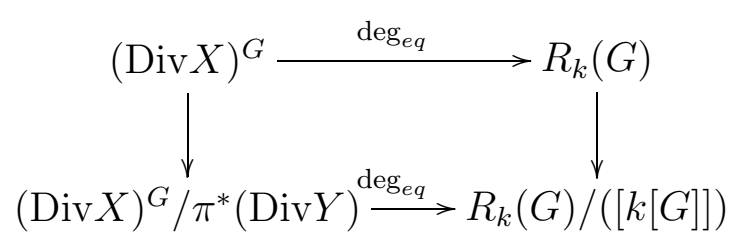

et de plus on a un isomorphisme canonique : 


$$
(\operatorname{Div} X)^{G} / \pi^{*}(\operatorname{Div} Y) \simeq \prod_{Q \in Y_{\text {ram }}} \mathbb{Z} /\left(e_{Q}\right)
$$

où $e_{Q}$ désigne l'indice de ramification associé au point $Q$ de $Y$.

\subsection{Formule de Riemann-Roch équivariante}

\subsubsection{Enoncé}

Théorème 3.11

Soit $\mathcal{L}$ un $G$-faisceau inversible sur $X$. On a l'égalité dans $R_{k}(G)$ :

$$
\chi(G, \mathcal{L})=\chi\left(G, \mathcal{O}_{X}\right)+\operatorname{deg}_{\text {eq }}(\mathcal{L})
$$

\subsubsection{Démonstration}

D'après la section 3.4.2, $\mathcal{L} \simeq_{G} \mathcal{L}_{X}(\Delta)$ pour un diviseur invariant $\Delta$. On suppose que le support de $\Delta$ contient une orbite $D=r D^{\prime}$ comme ci-dessus. On pose : $\Delta^{\prime}=\Delta-D$. On va montrer :

$$
\forall s \in \mathbb{Z} \quad \chi\left(G, \mathcal{L}_{X}\left(\Delta^{\prime}+(s+1) D^{\prime}\right)\right)-\chi\left(G, \mathcal{L}_{X}\left(\Delta^{\prime}+s D^{\prime}\right)\right)=\operatorname{Ind}_{G_{P}}^{G} \psi_{P}^{-(s+1)}
$$

ce qui suffira à démontrer le théorème, par récurrence. Soit $i$ l'immersion fermée : $D^{\prime} \rightarrow X$. On part de la suite exacte définissant la structure réduite de $D^{\prime}$ :

$$
0 \rightarrow \mathcal{L}_{X}\left(-D^{\prime}\right) \rightarrow \mathcal{O}_{X} \rightarrow i_{*}\left(\mathcal{O}_{D^{\prime}}\right) \rightarrow 0
$$

On tensorise par $\mathcal{L}_{X}\left(\Delta^{\prime}+(s+1) D^{\prime}\right)$ qui est localement libre, donc plat, ce qui donne :

$0 \rightarrow \mathcal{L}_{X}\left(\Delta^{\prime}+s D^{\prime}\right) \rightarrow \mathcal{L}_{X}\left(\Delta^{\prime}+(s+1) D^{\prime}\right) \rightarrow i_{*}\left(\mathcal{O}_{D^{\prime}}\right) \otimes_{\mathcal{O}_{X}} \mathcal{L}_{X}\left(\Delta^{\prime}+(s+1) D^{\prime}\right) \rightarrow 0$

Posons $\mathcal{S}=i_{*}\left(\mathcal{O}_{D^{\prime}}\right) \otimes_{\mathcal{O}_{X}} \mathcal{L}_{X}\left(\Delta^{\prime}+(s+1) D^{\prime}\right)$.

L'égalite (3.1) découle alors de

$$
\chi(G, \mathcal{S})=\operatorname{Ind}_{G_{P}}^{G} \psi_{P}^{-(s+1)} .
$$

Le calcul (3.2) résultera du lemme suivant.

\section{Lemme 3.12}

Soit $\mathcal{R}$ un $G$-faisceau de torsion sur $X$, à support dans $D^{\prime}=\sum_{\sigma \in G \backslash G_{P}} P^{\sigma}$. Si $\mathcal{R}=i_{*}\left(\mathcal{R}^{\prime}\right)$ où $i: D^{\prime} \rightarrow X$ est l'immersion fermée, on a :

$$
\chi(G, \mathcal{R})=\operatorname{Ind}_{G_{P}}^{G} \mathcal{R}_{P}^{\prime}
$$




\section{Démonstration :}

D'après la proposition 2.22 l'immersion fermée $i: D^{\prime} \rightarrow X$ induit un morphisme $K\left(G, D^{\prime}\right) \rightarrow K(G, X)$ qui commute avec les caractéristiques d'Euler équivariantes. D'autre part, $D^{\prime}=G \times^{G_{P}} P$ ce qui donne d'après la proposition 2.24 un isomorphisme :

$$
K\left(G, D^{\prime}\right)=K\left(G, G \times_{G_{P}} P\right) \simeq K\left(G_{P}, P\right)
$$

On conclut en remarquant que cet isomorphisme commute également avec les caractéristiques d'Euler équivariantes, plus précisement, le diagramme suivant est commutatif :

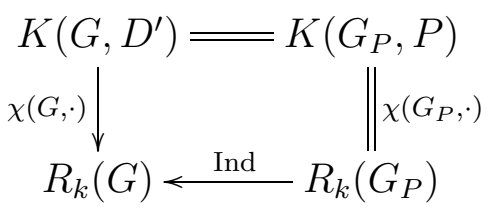

Revenons à la démonstration de l'égalité 3.2. Ici le faisceau de torsion considéré est $\mathcal{S}=i_{*}\left(\mathcal{O}_{D^{\prime}}\right) \otimes_{\mathcal{O}_{X}} \mathcal{L}_{X}\left(\Delta^{\prime}+(s+1) D^{\prime}\right)=i_{*}\left(\mathcal{S}^{\prime}\right)$ avec $\mathcal{S}^{\prime}=i^{*}\left(\mathcal{L}_{X}\left(\Delta^{\prime}+\right.\right.$ $\left.(s+1) D^{\prime}\right)$ ) donc $\mathcal{S}_{P}^{\prime}=\mathcal{O}_{P} \otimes_{\mathcal{O}_{X, P}} \mathfrak{m}_{P}^{-(s+1)}=\mathfrak{m}_{P}^{-(s+1)} / \mathfrak{m}_{P}^{-(s+2)}$, où $\mathfrak{m}_{P}$ désigne l'idéal maximal de $\mathcal{O}_{X, P}$. Donc $G_{P}$ agit par $\psi_{P}^{-(s+1)}$ sur $\mathcal{S}_{P}^{\prime}$, d'où le résultat.

\section{Remarque :}

Soit $r>0$. Le théorème 3.11 et la suite exacte

$$
0 \rightarrow \mathcal{L}_{X}\left(-r D^{\prime}\right) \rightarrow \mathcal{O}_{X} \rightarrow \mathcal{O}_{r D^{\prime}} \rightarrow 0
$$

pour $D^{\prime}$ orbite réduite montrent que l'on a :

$$
\operatorname{deg}_{e q}\left(-r D^{\prime}\right)=-\left[H^{0}\left(X, \mathcal{O}_{r D^{\prime}}\right)\right]
$$

où $\mathcal{O}_{r D^{\prime}}$ désigne le faisceau structurel du sous-schéma fermé défini par le faisceau d'idéaux $\mathcal{L}_{X}\left(-r D^{\prime}\right)$. On peut retrouver ce fait de manière élémentaire en filtrant le faisceau $\mathcal{O}_{r D^{\prime}}$.

\subsection{G-faisceaux inversibles de grand degré et ramification modérée}

Le théorème 3.11 permet de calculer la différence de deux caractéristiques d'Euler équivariantes. Cependant, si on en considère une seule, on ne dispose pour l'instant d'aucune d'expression absolue (i.e. totalement explicite en fonction des 
données de ramification). Le but de cette section est de montrer que si on suppose la ramification modérée, on peut donner une expression absolue de toute caractéristique d'Euler équivariante. Il suffit bien sûr de calculer l'une d'entre elles, les autres s'en déduisant grâce au théorème 3.11. On choisit naturellement de calculer celle du faisceau structurel (théorème 3.16). Celle-ci s'exprime en fonction d'un certain module de ramification qu'on introduit à la section 3.6.2.

Comme application de ce calcul, on donne un résultat (théorème 3.22) explicitant la structure de représentation de l'espace des sections globales d'un $G$-faisceau de grand degré.

Un des ingrédients essentiels de ces deux résultats est un critère de projectivité dû à Nakajima, dont on donne une démonstration directe.

\subsubsection{Projectivité de l'espace des sections globales d'un $G$-faisceau inversible de grand degré}

On rappelle tout d'abord le critère de projectivité classique suivant :

\section{Théorème 3.13}

Soit $G$ un groupe fini, $M$ un $k[G]$-module, $p$ la caractéristique de $k, G_{p}$ un $p$-Sylow de $G$. Les assertions suivantes sont équivalentes :

(i) $M$ est un $k[G]$-module projectif

(ii) $M_{\mid G_{p}}$ est un $k\left[G_{p}\right]$-module libre

(iii) $H^{1}\left(G_{p}, M_{\mid G_{p}}\right)=0$

\section{Démonstration :}

L'équivalence de (i) et (ii) est une conséquence d'un théorème de Green ([11], Corollary to Theorem 6). On peut en donner une preuve élémentaire n'utilisant que la notion de projectivité relative (voir par exemple [3]), comme suit. Le fait que $G_{p}$ est un $p$-Sylow de $G$ permet d'affirmer que $M$ est projectif relativement à

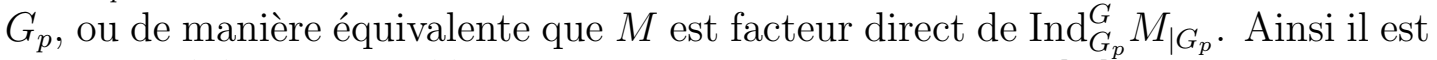
clair que (ii) implique (i). Réciproquement, si $M$ est un $k[G]$-module projectif, $M_{\mid G_{p}}$ est un $k\left[G_{p}\right]$-module projectif, ce qui équivaut au fait d'être libre, car $G_{p}$ est un $p$-groupe. Pour l'équivalence de (ii) et (iii), voir par exemple [3], Proposition 3.14.4.

On en déduit une nouvelle preuve du critère suivant :

\section{Corollaire 3.14 (Nakajima)}

Soit $X$ une courbe munie d'une action d'un groupe fini $G$. Si l'action de $X$ sur $G$ est modérée alors pour tout $G$-faisceau inversible $\mathcal{L}$ sur $X$ vérifiant $\operatorname{deg}(\mathcal{L})>$ $2 g_{X}-2$ l'espace $H^{0}(X, \mathcal{L})$ est $k[G]$-projectif.

\section{Démonstration :}

On note $\varpi: X \rightarrow Y_{p}=X / G_{p}$ le quotient. Les deux suites exactes fondamentales s'écrivent ici : 


$$
\begin{gathered}
0 \rightarrow H^{1}\left(G_{p}, \Gamma_{X}(\mathcal{L})\right) \rightarrow H^{1}\left(X, G_{p}, \mathcal{L}\right) \rightarrow 0 \\
0 \rightarrow H^{1}\left(Y_{p}, \varpi_{*}^{G_{p}} \mathcal{L}\right) \rightarrow H^{1}\left(X, G_{p}, \mathcal{L}\right) \rightarrow \Gamma_{Y_{p}}\left(R^{1} \varpi_{*}^{G_{p}} \mathcal{L}\right) \rightarrow 0
\end{gathered}
$$

L'hypothèse de modération implique que $G_{p}$ agit librement sur $X$, ce dont on déduit $R^{1} \varpi_{*}^{G_{p}} \mathcal{L}=0$ d'une part (d'après [13] $\mathrm{V} \S 2$ ), et $H^{1}\left(Y_{p}, \varpi_{*}^{G_{p}} \mathcal{L}\right)=0$ (pour des raisons de degré) d'autre part. Les deux suites exactes ci-dessus et le théorème 3.13 impliquent la conclusion.

\section{Remarque :}

Le corollaire 3.14 est en quelque sorte optimal, pour deux raisons. La première est que la situation pour des faisceaux de bas degré est beaucoup plus délicate (on peut voir par exemple que même si $X$ admet une action libre d'un $p$-groupe $H^{0}\left(X, \Omega_{X}\right)$ n'est jamais projectif). La deuxième raison est que la réciproque de ce corollaire est vraie, comme l'a noté Nakajima ([26], Theorem 2). On ne se servira pas de cette réciproque ici, mais voici brièvement une idée de preuve. Supposons l'action de $G$ sur $X$ sauvagement ramifiée. D'après les deux suites exactes fondamentales, il suffit d'exhiber un $\mathcal{L}$ tel que $\operatorname{deg} \mathcal{L}>2 g_{X}-2$ et $H^{1}\left(Y_{p}, \varpi_{*}^{G_{p}} \mathcal{L}\right) \neq 0$. Si $S$ est un ensemble $G$-stable de points de $X$ contenant le lieu de ramification de l'action, le faisceau $\Omega_{X}(S)$ est un candidat naturel (voir [16], Theorem 1).

\subsubsection{Module de ramification}

Par la suite on notera $\Gamma_{G}$ le module de ramification de $X$ pour l'action de $G$ introduit par Kani et Nakajima ([16],[25]). Rappelons en la définition.

Définition 3.15

On appelle module de ramification de $X$ pour l'action de $G$ le $k[G]$-module :

$$
\Gamma_{G}=\sum_{P \in X} \operatorname{Ind}_{G_{P}}^{G} \sum_{l=1}^{e_{P}-1} l \psi_{P}^{l}
$$

\subsubsection{Formule de Hurwitz équivariante}

\section{Théorème 3.16}

Soit $X$ une courbe projective et lisse munie d'une action modérée de $G$.

Il existe un unique $k[G]$-module $\widetilde{\Gamma}_{G}$ tel que $\widetilde{\Gamma}_{G}^{\oplus \# G}=\Gamma_{G}$.

On a l'égalité suivante dans $R_{k}(G)$ :

$$
\chi\left(G, \mathcal{O}_{X}\right)=\chi\left(\mathcal{O}_{Y}\right) \cdot[k[G]]-\left[\widetilde{\Gamma}_{G}\right]
$$




\subsubsection{Démonstration}

\section{Introduction}

$\mathrm{Au} \mathrm{vu} \mathrm{du} \mathrm{théorème} \mathrm{3.11,} \mathrm{il} \mathrm{est} \mathrm{clair} \mathrm{que} \mathrm{le} \mathrm{calcul} \mathrm{absolu} \mathrm{de} \mathrm{la} \mathrm{caractéristique}$ d'Euler équivariante de n'importe quel $G$-faisceau inversible permet de déduire le théorème 3.16. Ainsi, on peut conclure très rapidement en employant un résultat de Kani explicitant la structure de l'espace des sections globales d'un faisceau de différentielles logarithmiques sur la courbe ([16], Theorem 2) ou un résultat plus général de Nakajima ([25], Theorem 2). Cependant, on donne ici une preuve indépendante dont le principe est, grâce à des réductions successives, de pouvoir appliquer la formule de Lefschetz ([2] ou [15],VI,§9).

\section{Première réduction}

On va tout d'abord montrer qu'il suffit de prouver l'égalité suivante dans $R_{k}(G)$ :

$$
\# G\left(\chi\left(\mathcal{O}_{Y}\right) \cdot[k[G]]-\chi\left(G, \mathcal{O}_{X}\right)\right)=\left[\Gamma_{G}\right]
$$

On suppose donc l'égalité 3.3 vérifiée, et on va en déduire le théorème 3.16. Comme $R_{k}(G)$ est le groupe abélien libre engendré par les classes d'isomorphismes de $k[G]$-modules simples il suffit de démontrer :

$$
\exists ! \widetilde{\Gamma}_{G} / \widetilde{\Gamma}_{G}^{\oplus \# G} \simeq \Gamma_{G}
$$

On commence par noter que $\Gamma_{G}$ est un $k[G]$-module projectif, comme il résulte du lemme suivant.

\section{Lemme 3.17}

Soit $H$ un sous-groupe de $G$ tel que la caractéristique de $k$ ne divise pas \# $H$ et $M$ un $k[H]$-module de type fini. Alors $\operatorname{Ind}_{H}^{G} M$ est un $k[G]$-module projectif.

\section{Démonstration :}

$k[H]$ est semi-simple, donc $M$ est projectif, et comme le foncteur $\left(k[G] \otimes_{k[H]}-\right)$ commute aux sommes directes et envoie libres sur libres on a le résultat annoncé.

L'unicité dans 3.4 est à présent claire, en effet si $\widetilde{\Gamma}_{G}$ existe il est nécessairement projectif (comme facteur direct d'un module projectif), or l'égalité 3.4 détermine uniquement son caractère de Brauer, donc sa classe d'isomorphisme. Pour l'existence de $\widetilde{\Gamma}_{G}$ on va se servir du lemme suivant. Les notations sont celles de [31], partie III, en particulier $P_{k}(G)$ désigne le groupe de Grothendieck des $k[G]$ modules projectifs de type fini. 


\section{Lemme 3.18}

Soit $\mathcal{L}$ un $G$-faisceau inversible sur une courbe $X$ munie d'une action modérée. $\chi(G, \mathcal{L})$ est dans l'image de l'homomorphisme de Cartan $c: P_{k}(G) \rightarrow R_{k}(G)$.

\section{Démonstration :}

D'après le lemme 3.17, l'hypothèse de modération implique que pour tout $G$ faisceau inversible $\mathcal{L}, \operatorname{deg}_{\text {eq }} \mathcal{L}$ est dans l'image de $c$. D'après le théorème 3.11 il suffit donc de prouver le lemme pour un $G$-faisceau inversible quelconque. En appliquant le corollaire 3.14, on voit que n'importe quel $G$-faisceau de degré strictement supérieur à $2 g_{X}-2$ convient.

\section{Remarques :}

1. Ce lemme implique que $\chi\left(G, \mathcal{O}_{X}\right)$ est dans l'image de $c$, ce qui ne semble pas évident de prime abord.

2. Le résultat du lemme est valable pour un $G$-faisceau cohérent quelconque, comme on peut le déduire du théorème de structure de $K(G, X)$ qu'on verra verra par la suite, ou bien de [25], Theorem 1.

On déduit du lemme 3.18 et de l'égalité 3.4 qu'il existe un élément $x$ de $P_{k}(G)$ tel que l'égalité $\# G \cdot x=\left[\Gamma_{G}\right]$ tienne dans $P_{k}(G)$. Comme $P_{k}(G)$ est le groupe abélien libre engendré par les classes d'isomorphismes de $k[G]$-modules projectifs indécomposables, on peut écrire $x$ comme une somme de telles classes à coefficients entiers, qui doivent être positifs, puisque $\Gamma_{G}$ est une représentation de $G$. Il existe donc un module projectif $M$ tel que $\# G \cdot[M]=\left[\Gamma_{G}\right]$ dans $P_{k}(G)$, ce qui équivaut, puisque les deux modules sont projectifs, à $M^{\oplus \# G}=\Gamma_{G}$. Ceci conclut la démonstration du théorème 3.16, modulo la première réduction.

\section{Deuxième réduction}

Dans ce paragraphe on va voir qu'il suffit de montrer l'égalité 3.3 dans le cas où la courbe $X$ est munie d'une action d'un groupe cyclique d'ordre premier à la caractéristique $p$ de $k$.

Supposons donc que c'est le cas, et montrons que l'égalité 3.3 est valable dans le cas général. Pour cela, notons $x=\# G\left(\chi\left(\mathcal{O}_{Y}\right) \cdot[k[G]]-\chi\left(G, \mathcal{O}_{X}\right)\right), y=\left[\Gamma_{G}\right]$, et $\phi_{x}, \phi_{y}$ les caractères de Brauer associés. On veut montrer $x=y$, ce qui équivaut à $\phi_{x}=\phi_{y}$, ou encore au fait que pour tout sous-groupe cyclique $H$ de $G$ d'ordre

premier à $p$ on ait $\phi_{x \mid H}=\phi_{y \mid H}$. Ceci résultera de la formule de Hurwitz classique et des deux lemmes suivants.

\section{Lemme 3.19}

Soit $\mathcal{F}$ un $G$-faisceau.

$$
\chi(G, \mathcal{F})_{\mid H}=\chi(H, \mathcal{F})
$$




\section{Démonstration :}

Analogue à la démonstration de la proposition 2.4.

\section{Lemme 3.20}

$$
\left[\Gamma_{G}\right]_{\mid H} \equiv[G: H] \cdot\left[\Gamma_{H}\right] \bmod [k[H]]
$$

\section{Démonstration :}

Notons $Z$ la courbe quotient $Z=X / H$, on note $P, Q$ et $R$ des points quelconques de $X, Y$ et $Z$.

On commence par exprimer $\Gamma_{G}$ en fonction d'une somme sur $Q$. Pour cela on remarque que d'après la proposition 3.6 (iii), la quantité $\operatorname{Ind}_{G_{P}}^{G} \sum_{l=1}^{e_{P}-1} l \psi_{P}^{l}$ est indépendante de $P$. On peut donc écrire :

$$
\Gamma_{G}=\sum_{Q \in Y} \frac{\# G}{e(\tilde{Q} / Q)} \sum_{l=1}^{e(\tilde{Q} / Q)-1} l \operatorname{Ind}_{G_{\tilde{Q}}}^{G} \psi_{\tilde{Q}}^{l}
$$

où $\tilde{Q}$ est un relèvement arbitraire de $Q$ par $\pi: X \rightarrow Y$. On a bien sûr une formule analogue pour $\Gamma_{H}$, en fonction des caractères de ramification relatifs à $X \rightarrow X / H$, qu'on note $\left(\phi_{P}\right)_{P \in X}$.

Pour calculer $\Gamma_{G \mid H}$ on se sert de l'expression ci-dessus et de la formule de Mackey qui permet d'affirmer :

$$
\left(\operatorname{Ind}_{G_{\tilde{Q}}}^{G} \psi_{\tilde{Q}}^{l}\right)_{\mid H}=\sum_{R \rightarrow Q} \operatorname{Ind}_{H_{\tilde{R}}}^{H} \phi_{\tilde{R}}^{l}
$$

ce dont on déduit :

$$
\left[\sum_{l=1}^{e(\tilde{Q} / Q)-1} l \operatorname{Ind}_{G_{\tilde{Q}}}^{G} \psi_{\tilde{Q}}^{l}\right]_{\mid H} \equiv\left[\sum_{R \rightarrow Q} e(R / Q) \sum_{l=1}^{e(\tilde{R} / R)-1} l \operatorname{Ind}_{H_{\tilde{R}}}^{H} \phi_{\tilde{R}}^{l}\right] \quad \bmod [k[H]]
$$

Le lemme se déduit de cette dernière expression en sommant sur $Q$.

\section{Démonstration de l'égalité 3.3 dans le cas cyclique réductif}

On suppose $X$ munie d'une action d'une groupe $G$ cyclique d'ordre $n$ premier à la caractéristique de $k$. On note comme ci-dessus $x=\# G\left(\chi\left(\mathcal{O}_{Y}\right) \cdot[k[G]]-\right.$ $\left.\chi\left(G, \mathcal{O}_{X}\right)\right), y=\left[\Gamma_{G}\right]$. Comme $k[G]$ est semi-simple, on peut utiliser la théorie classique des caractères pour montrer que $x=y$ dans $R_{k}(G)$. On note $\chi_{x}, \chi_{y}$ les caractères de Frobénius associés à $x$ et $y$. 
Soit $\sigma$ un élémet de $g$. En procédant comme dans le paragraphe précédent on peut supposer que $\sigma$ est un générateur de $G$. On fixe $\zeta$ une racine primitive $n$-ième de l'unité. Si $P$ appartient à $X^{\sigma}$ on note $n_{P}$ l'entier, bien défini modulo $n$, tel que $\psi_{P}(\sigma)=\zeta^{n_{P}}$.

Pour calculer $\chi_{x}(\sigma)$ on applique la formule des points fixes de Lefschetz ([2] ou [15],VI,§9) au faisceau structurel ce qui donne :

$$
\chi_{x}(\sigma)=n \sum_{P \in X^{\sigma}} \frac{1}{\zeta^{n_{P}}-1}
$$

Dans le calcul de $\chi_{y}(\sigma)$, seuls interviennent les points totalement ramifiés, comme le montre le lemme suivant.

\section{Lemme 3.21}

Si $P \notin X^{\sigma}$ et $l \in \mathbb{Z}$ alors $\left(\operatorname{Ind}_{G_{P}}^{G} \psi_{P}^{l}\right)(\sigma)=0$

\section{Démonstration :}

Soit $\chi$ le générateur de $\hat{G}$ défini par : $\chi(\sigma)=\zeta$. Comme la restriction $\hat{G} \rightarrow \hat{G}_{P}$ est surjective on peut trouver un entier $n_{P}$ tel que $\psi_{P}=\chi_{\mid G_{P}}^{n_{P}}$. Il vient alors :

$$
\operatorname{Ind}_{G_{P}}^{G} \psi_{P}^{l}=\operatorname{Ind}_{G_{P}}^{G} \chi_{\mid G_{P}}^{l n_{P}}=\chi^{\prime} \cdot \chi^{l n_{P}}
$$

où $\chi^{\prime}$ est la représentation de permutation associée à l'action de $G$ sur $G_{P}$. Mais comme $P \notin X^{\sigma}$ et $G$ est cyclique, $\sigma$ ne fixe aucun des conjugués de $P$, et $\chi^{\prime}(\sigma)=0$.

On déduit immédiatement du lemme :

$$
\chi_{y}(\sigma)=\sum_{P \in X^{\sigma}} \sum_{l=1}^{n-1} l \zeta^{l n_{P}}
$$

et l'égalité $\chi_{x}(\sigma)=\chi_{y}(\sigma)$ découle de l'identité formelle :

$$
\frac{n}{\omega-1}=\sum_{l=1}^{n-1} l \omega^{l}
$$

valable pour toute racine $n$-ième de l'unité $\omega$ différente de 1 .

\section{Remarques :}

1. Le module de ramification $\Gamma_{G}$ a été introduit simultanément par Nakajima ([25]) et Kani ([16]). Comme le soulignent ces deux auteurs, le fait que $\Gamma_{G}$ soit divisible par \# $G$ constitue une relation globale remarquable entre les caractères de ramification. 
2. On peut adapter la preuve ci-dessus pour donner une deuxième démonstration du théorème 3.11 dans le cas modéré. Pour cela, on applique la formule des points fixes de Lefschetz à un $G$-faisceau inversible quelconque. On note que la formule de Mackey implique

$$
\left(\operatorname{deg}_{e q}(\mathcal{L})\right)_{\mid H}=\operatorname{deg}_{e q}\left(\mathcal{L}_{\mid H}\right)
$$

\subsubsection{Structure de représentation de l'espace des sections globales d'un $G$-faisceau inversible de grand degré}

Dans ce paragraphe on va montrer que les théorèmes 3.11 et 3.16 permettent de déterminer explicitement la structure galoisienne de l'espace des sections globales d'un $G$-faisceau inversible de grand degré. Il est clair que ces deux théorèmes déterminent le caractère de Brauer de $H^{0}(X, \mathcal{L})$ pour tout $G$-faisceau $\mathcal{L}$ inversible de degré de strictement supérieur à $2 g_{X}-2$. Dans le cas réductif, ceci détermine la structure de représentation de cet espace. Dans le cas non réductif, le caractère de Brauer d'une représentation ne la détermine pas toujours à isomorphisme près. Il faut donc raffiner la démarche précédente. Le corollaire suivant nous affirme que c'est possible lorsque l'action est modérée.

\section{Corollaire 3.22}

Soit $X$ une courbe projective et lisse munie d'une action modérée de $G$. Soit $\mathcal{L}$ un $G$-faisceau inversible sur $X$, tel que $\operatorname{deg} \mathcal{L}>2 g_{X}-2$. On a l'égalité suivante dans $P_{k}[G]$ :

$$
\left[H^{0}(X, \mathcal{L})\right]=\chi\left(\mathcal{O}_{Y}\right)[k[G]]-\left[\widetilde{\Gamma}_{G}\right]+\operatorname{deg}_{e q} \mathcal{L}
$$

\section{Démonstration :}

D'après les théorèmes 3.11 et 3.16 l'égalité est vraie dans $R_{k}[G]$. Pour justifier le corollaire, on peut invoquer l'injectivité de l'homomorphisme de Cartan à condition de montrer que tous les modules intervenant sont projectifs. La projectivité de $\widetilde{\Gamma}_{G}$ et de $\operatorname{deg}_{e q} \mathcal{L}$ résulte du lemme 3.17. La projectivité de $H^{0}(X, \mathcal{L})$ découle du corollaire 3.14.

\section{Remarque :}

D'après le théorème de Krull-Schmidt, le corollaire 3.22 caractérise complètement la structure de $H^{0}(X, \mathcal{L})$ comme représentation de $G$. Ce résultat est une version explicite de [26], Theorem 2. 


\subsection{Le problème de la structure de l'espace des sections globales du faisceau des différentielles}

\subsubsection{Enoncé du problème}

Il s'agit de résoudre le problème de Chevalley-Weil dans le cas modulaire, autrement dit, de déterminer la structure de $H^{0}\left(X, \Omega_{X}\right)$ comme $k[G]$-module lorsque $p$ divise l'ordre de $G$. Plus précisement, on demande d'écrire $H^{0}\left(X, \Omega_{X}\right)$ comme somme directe $k[G]$-modules indécomposables (cela suppose que la théorie de la représentation modulaire de $G$ soit bien comprise). Kani a donné une réponse satisfaisante à ce problème dans le cas où l'action est modérée (voir [16] et le paragraphe ci-dessous). Le problème est encore ouvert dans le cas d'une action sauvage, bien que résolu dans le cas $p$-cyclique (voir [35]) et certaines $p$-extensions abéliennes élémentaires (voir [29]). On se contente ici de donner une relation reliant dans le cas général le caractère de Brauer de $H^{0}\left(X, \Omega_{X}\right)$ à celui des caractères d'Artin de l'extension.

\subsubsection{Degré équivariant du faisceau des différentielles et caractère d'Artin}

Rappelons brièvement commet on définit le caractère d'Artin associé à la $G$ courbe $X$. Les notations sont inspirées de [30], chapitre VI, §4. Soit $P$ un point de $X$, on définit une fonction centrale $a_{P}$ sur $G_{P}$ en posant pour $g \neq 1$ $a_{P}(g)=-i_{P}(g)$ avec $i_{P}(g)=v_{P}\left(u_{p}^{g}-u_{P}\right)$, où $u_{P}$ est une uniformisante de $X$ en $P$, et $a_{G}(1)$ tel que $\left\langle a_{G}, 1>=0\right.$. C'est en fait un caractère de $G_{P}$, et pour $Q$ un point de $Y$, dont un relèvement arbitraire par $\pi$ est $\tilde{Q}$, on pose $a_{Q}=\operatorname{Ind}_{G_{\tilde{Q}}}^{G} a_{\tilde{Q}}$, caractère qui est indépendant du choix de $\tilde{Q}$. Enfin on peut définir le caractère d'Artin associé à $X$ :

$$
a_{G, X}=\sum_{Q \in Y} a_{Q}
$$

On s'intéresse à la décomposition de ce caractère. Pour préciser ce que cela signifie, on reprend les notations de [31] pour la théorie de la représentation modulaire. En particulier $K$ désigne un corps de caractéristique 0 complet pour une valuation discrète et de corps résiduel $k$ (le corps de base de la courbe $X$ considérée). D'après [31], $\$ 19.2$, on peut choisir un tel $K$ tel que $a_{G, X}$ soit réalisable sur $K$ (par exemple l'anneau des vecteurs de Witt sur $k$ convient). On peut alors énoncer :

\section{Théorème 3.23}

L'image de $a_{G, X}$ par l'homomorphisme de décomposition $d: R_{K}(G) \rightarrow R_{k}(G)$ est égale à $\operatorname{deg}_{e q} \Omega_{X}-\left(2 g_{Y}-2\right)[k[G]]$. 


\section{Démonstration :}

Par commodité, on introduit le diviseur de ramification $R$ du morphisme $\pi$; plus précisement $R$ est le diviseur associé au faisceau d'idéaux $\pi^{*} \Omega_{Y} \otimes_{\mathcal{O}_{X}} \Omega_{X}^{-1}$. Comme son faisceau d'idéaux est un $G$-faisceau, c'est un diviseur $G$-invariant.

On va comparer les caractères de Brauer associés aux deux expressions à comparer. En ces termes, $d\left(a_{G, X}\right)$ s'interprète comme la restriction du caractère d'Artin aux $p^{\prime}$-éléments de $G$.

La comparaison de ces deux caractères de Brauer en $g=1$ est aisée, en effet en vertu de [30], Proposition 4, on a $a_{P}(1)=v_{P}(R)$, et il suffit alors d'appliquer la formule classique d'Hurwitz, qui affirme que $2 g_{X}-2=\# G\left(2 g_{Y}-2\right)+\operatorname{deg} R$.

On note de plus que $\Omega_{X} \equiv \mathcal{L}_{X}(R) \bmod \pi^{*}(\operatorname{Pic} Y)$, donc $\operatorname{deg}_{e q} \Omega_{X} \equiv \operatorname{deg}_{e q} R$ $\bmod [k[G]]$. Il suffit donc à présent de comparer les caractères de Brauer associés à $d\left(a_{G, X}\right)$ et à $\operatorname{deg}_{e q} R$ en les $p^{\prime}$-éléments de $G$.

Soit à présent $P$ un point de $X, g$ un $p^{\prime}$-élément non trivial de $G_{P}$, il suffit de montrer l'égalité de $a_{P}(g)=-1$ et de $\sum_{l=1}^{v_{P}(R)-1} \psi_{P}(g)^{-l}$. On note $G_{P, i}$ le $i$-ème groupe de ramification et $e_{P, 0}=\# G_{P, 0} / \# G_{P, 1}$ la $p^{\prime}$-partie de l'indice de ramification de $\pi$ en $P$. $\psi_{P}(g)$ est une racine $e_{P, 0}$-ème de l'unité et le lemme suivant suffit donc à conclure.

\section{Lemme 3.24}

Les coefficients du diviseur de ramification $R$ vérifient la congruence suivante en tout point $P$ :

$$
v_{P}(R) \equiv-1 \quad \bmod e_{P, 0}
$$

\section{Démonstration :}

Soit $\omega^{\prime}$ une forme différentielle méromorphe non nulle sur $Y$, alors $\omega=\pi^{*} \omega^{\prime}$ est une forme différentielle méromorphe non nulle sur $X$, et $\omega$ est de plus $G$ invariante, ce qui permet d'affirmer qu'on a un isomorphisme de $G$-faisceaux : $\Omega_{X} \simeq \mathcal{L}_{X}((\omega))$. On a donc $(\omega) \equiv R \bmod \pi^{*}(\operatorname{Div} Y)$, et donc en particulier $v_{p}(\omega) \equiv v_{P}(R) \bmod e_{P, 0}$. Mais à présent le lemme 3.6 (ii) donne $\psi_{P}(g)^{v_{P}(\omega)+1}=$ 1 pour tout $g$ dans $G_{P}$. En choisissant $g$ dans $G_{P}$ tel que sa classe dans $G_{P, 0} / G_{P, 1}$ soit génératrice, on obtient la congruence souhaitée.

\section{Remarque :}

On peut énoncer une propriété analogue (et équivalente) reliant la décomposition du caractère de Swan de l'extension à un faisceau de différentiellles logarithmiques le long du lieu de ramification.

\section{Corollaire 3.25}

Si l'action de $G$ sur $X$ est modérée on a l'égalité dans $R_{k}(G)$ :

$$
\operatorname{deg}_{e q} \Omega_{X}=\left(2 g_{Y}-2+\# Y_{\text {ram }}\right)[k[G]]-\left[k X_{\text {ram }}\right]
$$

où \# $Y_{\text {ram }}$ est le nombre d'orbites ramifiées de $X$ et $k X_{\text {ram }}$ est la représentation de permutation de $G$ associée à l'ensemble des points de ramification de $X$. 
Démonstration :

L'image par $d$ du caractère d'Artin s'écrit dans le cas modéré :

$$
d\left(a_{G, X}\right)=\sum_{Q \in Y} \operatorname{Ind}_{G_{\tilde{Q}}}^{G}\left(\left[k\left[G_{\tilde{Q}}\right]\right]-[k]\right)
$$

(voir par exemple [31], chapitre 19, §19.1). Le théorème 3.23 permet alors de conclure.

\subsubsection{Structure de l'espace des sections globales du fais- ceau des différentielles dans le cas modéré}

On trace tout d'abord les grandes lignes de la stratégie de Kani dans [16]. Celuici a eu l'idée d'utiliser un faisceau $\Omega_{X}(S)$ de différentielles logarithmiques le long d'un ensemble $G$-stable non vide $S$ contenant le lieu de ramification de $\pi$. L'intérêt de ce faisceau vient du fait que la suite exacte longue de cohomologie associée à la suite exacte courte de faisceaux :

$$
0 \rightarrow \Omega_{X} \rightarrow \Omega_{X}(S) \rightarrow \mathcal{O}_{S} \rightarrow 0
$$

détermine complètement la structure de $H^{0}\left(X, \Omega_{X}\right)$ (voir [16], §5). Il reste donc à expliciter la structure de $H^{0}\left(X, \Omega_{X}(S)\right)$. On va montrer que le résultat suivant est une conséquence directe des théorèmes 3.11 et 3.16 .

\section{Théorème 3.26 (Kani)}

Si l'action de $G$ sur $X$ est modérée on a un isomorphisme de $k[G]$-modules :

$$
H^{0}\left(X, \Omega_{X}(S)\right) \oplus \widetilde{\Gamma}_{G} \simeq k[G]^{\oplus g_{Y}-1+\#(S / G)}
$$

\section{Démonstration :}

Comme $S$ est non vide, le corollaire 3.14 permet d'affirmer que $H^{0}\left(X, \Omega_{X}(S)\right)$ est projectif. Il suffit donc de prouver l'égalité du théorème dans $R_{k}(G)$. Le fait que $H^{1}\left(X, \Omega_{X}(S)\right)=0$ et les théorèmes 3.11 et 3.16 montrent de plus que cette égalité équivaut à :

$$
\operatorname{deg}_{e q} \Omega_{X}(S)=\left(2 g_{Y}-2+\#(S / G)\right) \cdot[k[G]]
$$

Cette dernière égalité découle des deux faits suivants. En prenant les caractéristiques d'Euler équivariantes sur la suite exacte 3.5 et en appliquant une nouvelle fois le théorème 3.11 on obtient $\operatorname{deg}_{e q} \Omega_{X}(S)=\operatorname{deg}_{e q} \Omega_{X}+[k S]$ où $k S$ est la représentation de permutation associée au $G$-ensemble $S$. D'autre part, $\operatorname{deg}_{e q} \Omega_{X}$ se calcule à l'aide du corollaire 3.25. 


\subsubsection{Dualité équivariante et formule à la Weil}

Dans le cas où l'action est sauvage, la démarche ci-dessus échoue, faute de projectivité. On peut cependant donner une relation concernant le caractère de Brauer de $H^{0}\left(X, \Omega_{X}\right)$. Pour cela on aura besoin du lemme suivant.

\section{Lemme 3.27}

Soit $\mathcal{F}$ un $G$-faisceau cohérent. On a un isomorphisme de $k[G]$-modules :

$$
H^{1}(X, \mathcal{F})^{\vee} \simeq H^{0}\left(X, \Omega_{X} \otimes_{\mathcal{O}_{X}} \check{\mathcal{F}}\right)
$$

\section{Démonstration :}

Le cup-produit fournit un accouplement :

$$
H^{0}\left(X, \Omega_{X} \otimes_{\mathcal{O}_{X}} \check{\mathcal{F}}\right) \times H^{1}(X, \mathcal{F}) \rightarrow H^{1}\left(X, \Omega_{X}\right)
$$

Cet accouplement est naturel, et commute donc à l'action de $G$. Il suffit donc de montrer que $H^{1}\left(X, \Omega_{X}\right)$ est isomorphe à la représentation triviale, qu'on note $k$. Pour cela on considère la suite exacte longue associée à la suite exacte courte 3.5 du paragraphe ci-dessus, qui donne en particulier :

$$
H^{0}\left(X, \Omega_{X}(S)\right) \rightarrow k S \rightarrow H^{1}\left(X, \Omega_{X}\right) \rightarrow 0
$$

Comme la somme des résidus d'une forme différentielle logarithmique est nulle, le $G$-morphisme trace $\operatorname{tr}: k S \rightarrow k$ se factorise en un $G$-morphisme $H^{1}\left(X, \Omega_{X}\right) \rightarrow k$ qui est en fait un isomorphisme.

\section{Théorème 3.28}

On a l'égalité dans $R_{k}(G)$ :

$$
\left[H^{0}\left(X, \Omega_{X}\right)\right]+\left[H^{0}\left(X, \Omega_{X}\right)^{\vee}\right]=2 \cdot[k]+\left(2 g_{Y}-2\right) \cdot[k[G]]+d\left(a_{G, X}\right)
$$

où $[k]$ désigne la classe de la représentation triviale de $G$.

\section{Démonstration :}

C'est une conséquence directe du théorème 3.11 appliqué à $\mathcal{L}=\Omega_{X}$, du théorème 3.16, du lemme ci-dessus (qui donne les isomorphismes $H^{1}\left(X, \mathcal{O}_{X}\right) \simeq H^{0}\left(X, \Omega_{X}\right)^{\vee}$ et $H^{1}\left(X, \Omega_{X}\right) \simeq k$ ) et du théorème 3.23.

\section{Remarques :}

1. Cette propriété a été énoncée par Kani ([16], page 200, Remark) avec annonce d'une preuve apparement jamais publiée.

2. Cette formule est un analogue cohérent de la formule de Weil (voir par exemple [30] chapitre VI $\S 4$ Proposition 7), avec laquelle il semble souhaitable de faire le lien. Nous ne savons pas s'il est possible de passer d'une formule à l'autre. 


\subsection{Conclusion}

Comme son analogue classique, le théorème 3.11 s'est avéré pratique dans bien des situations. D'autre part, la notion de degré équivariant a permis de formuler de manière concise quel type d'information un $G$-faisceau inversible véhicule. Contrairement au cas classique, une partie de cette information est de nature locale, par exemple le degré équivariant du faisceau modulo $[k[G]]$. D'autre part, un résultat de nature purement arithmétique comme le lemme 3.24 peut s'interpréter comme un calcul de degré équivariant. Ce calcul présente les difficultés habituelles d'un calcul de classe de Chern, autrement dit dans l'isomorphisme :

$$
\frac{(\operatorname{Div} X)^{G}}{\pi^{*}(\operatorname{Princ} Y)} \simeq \operatorname{Pic}_{G} X
$$

le morphisme direct est tout à fait explicite, mais le morphisme réciproque est bien plus mystérieux. Ces remarques incitent naturellement à approfondir le lien entre $G$-faisceaux et cycles, qui est au centre du chapitre suivant. 


\section{Chapitre 4}

\section{Cycles équivariants}

\subsection{Introduction}

Dans ce chapitre, on conserve les notations du chapitre précédent ; en particulier $X$ désignera une courbe projective lisse sur un corps algébriquement clos $k$, munie d'une action d'un groupe fini $G$. Pour des raisons techniques on aura à supposer que cette action est modérée. Le théorème 3.11 soulève la question : a-t-on un résultat équivalent pour un faisceau localement libre de rang quelconque ? Ceci nous amène naturellement à l'étude du groupe $K(G, X)$. Pour justifier cette étude, considérons le cas classique (i.e. non équivariant), on a alors un un isomorphisme de groupes remarquable :

$$
\begin{gathered}
\mathbb{Z} \oplus \operatorname{Pic} X \longrightarrow K(X) \\
(r, \mathcal{L}) \longrightarrow(r-1)\left[\mathcal{O}_{X}\right]+[\mathcal{L}] \\
(\operatorname{rk}(\mathcal{E}), \operatorname{det}(\mathcal{E})) \longleftrightarrow[\mathcal{E}]
\end{gathered}
$$

Comme nous l'a fait remarquer Bernhard Köck, cet isomorphisme permet de déduire la formule de Riemann-Roch en rang quelconque de la formule en rang 1. En combinant une étude de Pic $X$, on peut même en déduire la formule en rang 1. Il est donc légitime de penser qu'un théorème de structure concernant ce groupe permettrait de dériver formellement un théorème de type Riemann-Roch concernant les $G$-faisceaux.

Le problème de la structure de groupe $K(G, X)$ a été étudié par Ellingsrud et Lønsted ([20], [8]). Le théorème principal issu de cet étude ([8] Theorem 1.7) ne peut être considéré comme pleinement satisfaisant puisque dans le cas de l'action triviale, il ne se spécialise pas dans l'isomorphisme 4.1, mais en un l'identité de $K(X)$.

Que peut on modifier dans 4.1 pour espérer généraliser cette formule? Compte tenu du fait que $K(G, X)$ a une structure naturelle de $R_{k}(G)$-module, on peut s'interroger sur le fait que le facteur $\mathbb{Z}$ de la somme directe de 4.1 est bien pertinent 
dans le cas équivariant. Il y a-t-il une notion de rang équivariant ? Du point de vue de la $K$-théorie classique le morphisme rang peut être vu comme le morphisme induit en $K$-théorie par l'image réciproque de $\zeta \rightarrow X$ où $\zeta$ est le point générique de la courbe irréductible $X$. En $K$-théorie équivariante on doit donc s'intéresser à $K(G, \zeta)$, groupe qui classifie les $K(X) \circ G$ modules de dimension finie sur $K$. Or on a vu au paragraphe 2.1.3 que ceux ci sont classifiés par leur dimension, donc $K(G, \zeta) \simeq \mathbb{Z}$, et le rang équivariant est le même que le rang classique. On doit donc conserver le facteur $\mathbb{Z}$ dans la somme directe de 4.1 , et on note qu'on peut voir ce groupe comme $R_{k}(G)$-module via le morphisme d'anneaux $\operatorname{dim}: R_{k}(G) \rightarrow \mathbb{Z}$.

Une généralisation naturelle du deuxième terme de la somme directe de 4.1 , à savoir Pic $X$, est bien évidemment $\operatorname{Pic}_{G} X$, qu'on peut voir comme $R_{k}(G)$-module en faisant agir une représentation par son déterminant. Cependant l'analogue direct de l'isomorphisme classique, à savoir $\mathbb{Z} \oplus \mathrm{Pic}_{G} X \simeq K(G, X)$, s'avère être faux (comme déja mentionné dans l'introduction de [20]). Plus précisement, deux difficultés se présentent. La première est que la formule concernant le produit de deux $(G)$-faisceaux inversibles

$$
\left[\mathcal{L}_{1} \otimes_{\mathcal{O}_{X}} \mathcal{L}_{2}\right]-\left[\mathcal{L}_{1}\right]-\left[\mathcal{L}_{2}\right]+\left[\mathcal{O}_{X}\right]=0
$$

valable dans $K(X)$ n'est plus valable dans $K(G, X)$. Par conséquent, on n'a pas de morphisme naturel $\operatorname{Pic}_{G} X \rightarrow K(G, X)$. Une deuxième difficulté, encore plus importante, réside dans le fait que même d'un point de vue ensembliste l'application naturelle $\mathbb{Z} \oplus \operatorname{Pic}_{G} X \rightarrow K(G, X)$ n'est pas surjective. Autrement dit, les classes $\left[\mathcal{O}_{Y}\right]$ n'engendrent pas $K(G, X)$ comme groupe abélien lorsque $Y$ varie parmi les cycles $G$-invariants de $X$, contrairement à ce qui se passe dans le cas non équivariant ([21] $§ 2$ Theorem 2.2). Ce phénomène est résumé classiquement par la phrase : il n'y a pas assez de cycles $G$-invariants.

Pour remédier au problème ci-dessus, on remarque que si l'on s'autorise à multiplier les classes $\left[\mathcal{O}_{Y}\right]$ non pas seulement par des entiers, mais par des représentations, alors ces classes engendrent $K(G, X)$. Une démarche naturelle est donc de considérer un groupe $A^{1}(G, X)$ de 1-cycles dont les coefficients sont des représentations, modulo une relation généralisant l'équivalence rationelle des diviseurs, qu'on appelera l'équivalence $Y$-rationelle. Il se trouve que ce groupe sera l'analogue de Pic $X$ convenable pour généraliser l'isomorphisme 4.1 au cas équivariant.

L'application principale de cette généralisation est de permettre de définir formellement le déterminant équivariant d'un $G$-faisceau. Plus précisement on construit un morphisme $\operatorname{det}_{G}$ relevant le déterminant usuel : 


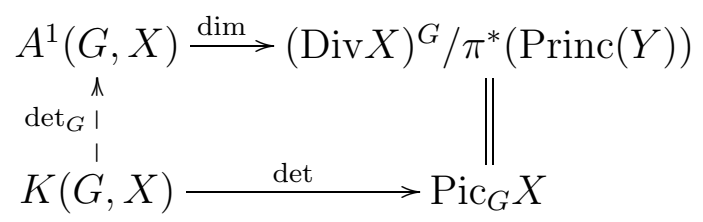

\subsection{Diviseurs équivariants}

\subsubsection{Définition et degré}

\section{Définition 4.1}

On appelle diviseur équivariant sur $X$ toute somme formelle de points de $X$ du type $D=\sum_{P \in X} V_{P} P$ vérifiant :

1. $V_{P}$ est une représentation virtuelle du stabilisateur $G_{P}$ de $P$, nulle sauf pour un nombre fini de points,

2. si $P^{\prime}=g P$ pour $g \in G$ alors $V_{P^{\prime}}=V_{P}^{g}$.

On dira que $D$ est de plus effectif si toutes les représentations $V_{P}$ sont des vraies représentations. On notera $\operatorname{Div}(G, X)$ le groupe abélien des diviseurs équivariants sur $X$.

Notation :

On notera $\operatorname{Ind}_{G_{P}}^{G} V_{P} P$ le diviseur $D=\sum_{g \in G \backslash G_{P}} V_{P}^{g} g P$, qu'on appelera orbite. Les orbites engendrent $\operatorname{Div}(G, X)$ comme groupe abélien.

\section{Définition $\mathbf{4 . 2}$}

On appelera $G$-degré le morphisme $\operatorname{deg}_{G}: \operatorname{Div}(G, X) \rightarrow R_{k}(G)$ qui à une orbite $\operatorname{Ind}_{G_{P}}^{G} V_{P} \cdot P$ associe la représentation $\operatorname{Ind}_{G_{P}}^{G} V_{P}$.

\subsubsection{Faisceau de torsion associé à un diviseur équivariant}

Un diviseur équivariant effectif détermine canoniquement un $G$-faisceau de torsion sur $X$ : le faisceau gratte-ciel associé (c'est plus précisement la somme des images directes des représentations $V_{P}$ par les flèches canoniques $\left.\operatorname{Spec}(k(P)) \rightarrow X\right)$. Puisque les diviseurs équivariants effectifs engendrent $\operatorname{Div}(G, X)$ comme groupe abélien, on définit ainsi un morphisme :

$$
\gamma: \operatorname{Div}(G, X) \rightarrow K(G, X)
$$

L'intérêt de ces faisceaux de torsion est d'avoir une caractéristique d'Euler équivariante facile à calculer :

\section{Lemme 4.3}

Soit $D$ un diviseur équivariant sur $X$. On a l'égalité dans $R_{k}(G)$ :

$$
\chi(G, \gamma(D))=\operatorname{deg}_{G} D
$$




\section{Démonstration :}

Soit $R$ le support de $D$. La formule à démontrer étant additive en $D$ on peut supposer que $R$ est restreint à une orbite. On peut alors écrire $D$ sous la forme $\operatorname{Ind}_{G_{P}}^{G} V_{P} P$. Notons $i: R \rightarrow X$ l'immersion fermée. On considère le diagramme commutatif :

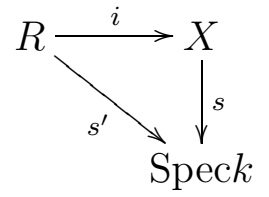

où $s$ et $s^{\prime}$ désignent les morphismes structurels. Par définition $\gamma(D)=i_{*}\left(\operatorname{Ind}_{G_{P}}^{G} V_{P} P\right)$ et $\chi(G,)=.s_{*}$. D'après le théorème $2.22 s_{*} \circ i_{*}=s_{*}^{\prime}$ d'où $\chi(G, \gamma(D))=s_{*}^{\prime}(D)=$ $\operatorname{deg}_{G} D$.

\subsubsection{Base en colimaçon}

On introduit dans ce paragraphe une base de $\operatorname{Div}(G, X)$ qui va être très utile par la suite, et dont l'importance est apparue au chapitre précédent. Dans la généralisation de l'isomorphisme (4.1) qu'on construit, ces diviseurs correspondront aux $G$-faisceaux inversibles sur $X$. Il suffit de donner une base de $R_{k}\left(G_{P}\right)$ pour un $P$ dans chaque orbite. On choisit la "base en colimaçon" $\left\{V_{P, k} \mid 0 \leq k \leq e_{P}-1\right\}$ avec $e_{P}=\# G_{P}$ et

$$
V_{P, k}=\sum_{l=0}^{k} \psi_{P}^{l}
$$

On dispose à présent d'une base $\left\{\operatorname{Ind}_{G_{P}}^{G} V_{P, k} P \mid P \in X / G ; 0 \leq k \leq e_{P}-1\right\}$ de $\operatorname{Div}(G, X)$ dont l'intérêt réside dans le lemme suivant :

\section{Lemme 4.4}

Soit $P$ un point de $X$, et $R$ le diviseur réduit à coefficients entiers $\operatorname{Ind}_{G_{P}}^{G} P$. On a l'égalité pour $0 \leq k \leq e_{P}-1$ :

$$
\gamma\left(\operatorname{Ind}_{G_{P}}^{G} V_{P, k} \cdot P\right)=\left[\mathcal{O}_{X}\right]-\left[\mathcal{L}_{X}(-(k+1) R)\right]
$$

\section{Démonstration :}

On reprend l'idée de la démonstration du théorème 3.11. Plus précisement on va utiliser la filtration :

$$
\mathcal{L}_{X}(-k R) \subseteq \mathcal{L}_{X}((-k+1) R) \subseteq \cdots \subseteq \mathcal{L}_{X}(-R) \subseteq \mathcal{O}_{X}
$$

Il suffit en effet clairement de montrer :

$$
\left[\mathcal{L}_{X}(-l R)\right]-\left[\mathcal{L}_{X}(-(l+1) R)\right]=\gamma\left(\operatorname{Ind}_{G_{P}}^{G} \psi_{P}^{l} P\right)
$$

On note $i: R \rightarrow X$ l'immersion fermée correspondant à $X$. La suite exacte 


$$
0 \rightarrow \mathcal{L}_{X}(-R) \rightarrow \mathcal{O}_{X} \rightarrow i_{*}\left(\mathcal{O}_{R}\right) \rightarrow 0
$$

tensorisée par $\mathcal{L}_{X}(-l R)$ donne :

$$
\left[\mathcal{L}_{X}(-l R)\right]-\left[\mathcal{L}_{X}(-(l+1) R)\right]=\left[i_{*}\left(\mathcal{O}_{R}\right) \otimes_{\mathcal{O}_{X}} \mathcal{L}_{X}(-l R)\right]
$$

or d'après la formule de projection : $i_{*}\left(\mathcal{O}_{R}\right) \otimes_{\mathcal{O}_{X}} \mathcal{L}_{X}(-l R) \simeq i_{*}\left(i^{*}\left(\mathcal{L}_{X}(-l R)\right)\right.$. De plus $i_{*}\left(i^{*}\left(\mathcal{L}_{X}(-l R)\right)_{P}=\mathfrak{m}_{P}^{l} / \mathfrak{m}_{P}^{l+1}\right.$, donc $G_{P}$ agit par $\psi_{P}^{l}$ sur cette fibre. L'équivalence entre $G_{P}$-modules sur $P$ et $G$-modules sur $R$ vue au paragraphe 2.6.5 permet de déduire l'égalité 4.2 et conclut la démonstration du lemme 4.4.

\subsection{Equivalence $Y$-rationelle}

Dans le chapitre précédent, on a vu que les faisceaux du type $\pi^{*} \mathcal{M}$ avaient un degré équivariant multiple de $[k[G]]$. La définition suivante introduit les diviseurs correspondants.

\section{Définition 4.5}

On définit le pull-back équivariant (par rapport au morphisme $\pi$ ) comme étant le morphisme $\pi_{G}^{*}: \operatorname{Div}(Y) \rightarrow \operatorname{Div}(G, X)$ vérifiant pour tout point $Q$ de $Y$ :

$$
\pi_{G}^{*}(Q)=\sum_{P \rightarrow Q} k\left[G_{P}\right] P
$$

Depuis le théorème 2.34 on sait que pour obtenir une bonne correspondance entre $G$-diviseurs et $G$-faisceaux il faut considérer les $G$-diviseurs modulo les diviseurs de fonctions $G$-invariantes, c'est ce qu'on appele ici l'équivalence $Y$ rationelle. Plus précisement :

\section{Définition 4.6}

Le groupe des diviseurs équivariants sur $X$ est défini par :

$$
A^{1}(G, X)=\frac{\operatorname{Div}(G, X)}{\pi_{G}^{*}(\operatorname{Princ}(Y))}
$$

où $\operatorname{Princ}(Y)$ est le groupe des diviseurs principaux sur $Y$.

On va à présent montrer que les notions de degré et de cycle associé à un diviseur ne dépendent que de la classe d'équivalence $Y$-rationelle de celui-ci. On commence par la notion de degré :

\section{Lemme 4.7}

Le diagramme suivant commute :

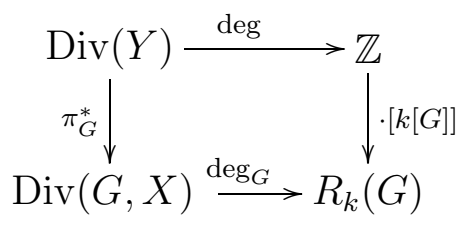




\section{Démonstration :}

Immédiat à partir des définitions.

On en déduit que le morphisme $\operatorname{deg}_{G}$ passe donc au quotient, comme l'indique la définition ci-après :

\section{Définition 4.8}

On appelle G-degré d'une classe de diviseurs équivariants (pour l'équivalence $Y$-rationelle) le degré de n'importe quel élément de cette classe. On note (abusivement) $\operatorname{deg}_{G}: A^{1}(G, X) \rightarrow R_{k}(G)$ le morphisme correspondant.

On montre à présent que la notion de cycle associé passe aussi au quotient. On note $\gamma: \operatorname{Div}(Y) \rightarrow K(Y)$ le morphisme défini par $\gamma(\delta)=\left[\mathcal{O}_{\delta}\right]$.

\section{Lemme 4.9}

Le diagramme suivant commute :

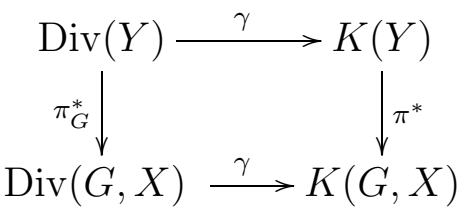

\section{Démonstration :}

Soit $Q$ un point de $Y$. Le lemme 4.4 donne

$$
\gamma\left(\pi_{G}^{*} Q\right)=\gamma\left(\operatorname{Ind}_{G_{\tilde{Q}}}^{G} k\left[G_{\tilde{Q}}\right] \tilde{Q}\right)=\left[\mathcal{O}_{X}\right]-\left[\mathcal{L}_{X}\left(\pi^{*}(-Q)\right)\right]
$$

or $\gamma(Q)=\left[\mathcal{O}_{Y}\right]-\left[\mathcal{L}_{Y}(-Q)\right]$, ce qui permet de conclure.

Ce lemme justifie la définition suivante.

\section{Définition 4.10}

On note $\gamma: A^{1}(G, X) \rightarrow K(G, X)$ l'application déduite de $\gamma: \operatorname{Div}(G, X) \rightarrow$ $K(G, X)$ par passage au quotient.

\subsection{Structure de groupe de $K(G, X)$}

\subsubsection{Enoncé}

\section{Théorème 4.11}

On a un isomorphisme de groupes :

$$
\begin{aligned}
\mathbb{Z} \oplus A^{1}(G, X) \longrightarrow K(G, X) & \longrightarrow \\
(r, D) \longrightarrow & \left.\longrightarrow \mathcal{O}_{X}\right]+\gamma(D)
\end{aligned}
$$




\subsubsection{Démonstration}

\section{Surjectivité}

On note tout d'abord que dans le cas classique, les images directes des faisceaux structurels des cycles engendrent $K(X)$ pour n'importe quelle variété projective $X$, voir [4], lemme 17 . On se contente ici de donner une démonstration d'une version équivariante de ce fait lorsque $X$ est une courbe, bien qu'une telle propriété doive être vraie en dimension quelconque. Soit $\mathcal{E}$ un $G$-faisceau localement libre sur $X$. Pour montrer que $[\mathcal{E}]$ est dans l'image du morphisme ci-dessus, on va d'abord montrer qu'on peut se ramener au cas où $[\mathcal{E}]$ est la classe d'un faisceau de torsion sur $X$. D'après la proposition 2.13 on a un morphisme injectif naturel $\pi^{*} \pi_{*}^{G} \mathcal{E} \rightarrow \mathcal{E}$. Notons $\mathcal{R}$ sa coimage, d'après le théorème 2.12 $\mathcal{E}$ et $\pi^{*} \pi_{*}^{G} \mathcal{E}$ sont localement libres de même rang, donc $\mathcal{R}$ est de torsion. De plus on a $[\mathcal{E}]=\left[\pi^{*} \pi_{*}^{G} \mathcal{E}\right]+[\mathcal{R}]$, donc pour effectuer la réduction annoncée, il suffit de montrer que $\left[\pi^{*} \pi_{*}^{G} \mathcal{E}\right]$ est dans l'image du morphisme du théorème cidessus. Or on sait que le théorème est vrai dans le cas non équivariant donc $\left[\pi_{*}^{G} \mathcal{E}\right]=\operatorname{rk} \mathcal{E}\left[\mathcal{O}_{Y}\right]+\gamma(\delta)$ pour un certain diviseur $\delta$ sur $Y$. Le lemme 4.9 donne alors $\left[\pi^{*} \pi_{*}^{G} \mathcal{E}\right]=\operatorname{rk} \mathcal{E}\left[\mathcal{O}_{X}\right]+\gamma\left(\pi^{*} \delta\right)$, ce qui achève le dévissage. Soit à présent $\mathcal{E}$ un faisceau de torsion sur $X$. Soit $R$ le support de $\mathcal{E}, i: R \rightarrow X$ l'immersion fermée corespondante, et $U=X-R$. En utilisant l'additivité de $\gamma$ on peut supposer que $R$ est réduit à une orbite. D'après le théorème 2.25 on a une suite exacte :

$$
K(G, R) \stackrel{i_{*}}{\longrightarrow} K(G, X) \stackrel{\text { res }}{\longrightarrow} K(G, U) \longrightarrow 0
$$

Par hypothèse, $\operatorname{res}(\mathcal{E})=0$, ce qui montre que $\mathcal{E}$ s'écrit $i_{*}[x]$ pour un $[x]$ dans $K(G, R)$. Mais si $P$ est un point dans l'orbite $R$, le groupe $K(G, R)$ s'identifie à $K\left(G_{P}, P\right)=R_{k}\left(G_{P}\right)$, ce qui permet de considérer $[x]$ comme un diviseur équivariant à support dans $R$, qui est l'antécédent de $[\mathcal{E}]$ recherché.

\section{Injectivité}

Soit $(r, D)$ dans $\mathbb{Z} \oplus A^{1}(G, X)$ tel que $r\left[\mathcal{O}_{X}\right]+\gamma(D)=0$. En appliquant le morphisme composé

$$
K(G, X) \stackrel{\text { oubli }}{\longrightarrow} K(X) \stackrel{\mathrm{rk}}{\longrightarrow} \mathbb{Z}
$$

on obtient $r=0$. Pour montrer que $D=0$ on va utiliser le lemme suivant.

\section{Lemme 4.12}

Pour tout point $P$ de $X$, on note $i_{P}: G \times{ }^{G_{P}} P \rightarrow X$ la $G$-immersion fermée correspondante, $i_{P_{*}}: R_{k}\left(G_{P}\right) \rightarrow K(G, X)$ et $i_{P}^{*}: K(G, X) \rightarrow R_{k}\left(G_{P}\right)$ les morphismes en $K$-théorie obtenus en identifiant $K\left(G, G \times{ }^{G_{P}} P\right)$ à $R_{k}\left(G_{P}\right)$. Alors :

(i) $i_{P^{\prime}}^{*} \circ i_{P *}=0$ si $P$ et $P^{\prime}$ ne sont pas conjugués sous $G$,

(ii) $i_{P}^{*} \circ i_{P *}\left(\psi_{P}^{k}\right)=\psi_{P}^{k}-\psi_{P}^{k+1}$ pour $k=0, \cdots, e_{P}-1$. 


\section{Démonstration :}

Dans la base en colimaçon relative à $P, \psi_{P}^{k}$ s'écrit $\left[V_{P, k}\right]-\left[V_{P, k-1}\right]$, et donc d'après le lemme 4.4:

$$
i_{P *}\left(\psi_{P}^{k}\right)=\left[\mathcal{L}_{X}(-k R)\right]-\left[\mathcal{L}_{X}(-(k+1) R)\right]
$$

avec $R=\operatorname{Ind}_{G_{P}}^{G} P$. On conclut en utilisant la proposition 2.18 qui affirme que le foncteur $i_{P}^{*}: \operatorname{Proj}(G, X) \rightarrow \operatorname{Proj}\left(G, G \times{ }^{G_{P}} P\right)$ est exact (ce qui donne pour un $G$-faisceau localement libre $\left.\mathcal{E}: i_{P}^{*}([\mathcal{E}])=\left[i_{P}^{*}(\mathcal{E})\right]\right)$.

On peut à présent terminer la démonstration du théorème 4.11. On dispose d'un diviseur équivariant $D$ tel que $\gamma(D)=0$. En décomposant le coefficient de $D$ en $P$ par rapport aux $\psi_{P}^{k}$, et en utilisant le lemme 4.12, on voit ce coefficient est un multiple de $\left[k\left[G_{P}\right]\right]$. Donc $D=\pi_{G}^{*}(\delta)$ pour un diviseur $\delta$ sur $Y$. En utilisant à présent le lemme 4.9 on obtient $\pi^{*}(\gamma(\delta))=0$. Mais d'après la proposition $2.28 \pi^{*}$ est injectif, donc $\gamma(\delta)=0$. Comme $\mathbb{Z} \oplus A^{1}(Y) \simeq K(Y)$ le morphisme $\gamma: A^{1}(Y) \rightarrow K(Y)$ est injectif, ce qui permet d'affirmer que $\delta$ est un diviseur principal sur $Y$, et donc que $D=0$ dans $A^{1}(G, X)$.

\section{Remarque :}

On peut raccourcir la démonstration précédente en utilisant les groupes de $K$ théorie supérieurs. Plus précisement, si $R$ une sous-variété fermée stricte de $X$ contenant le lieu de ramification de $\pi, B$ l'image de $X$ par $\pi$, vue comme sousvariété de $Y, U=X-R, V=Y-B$, on peut utiliser le diagramme commutatif suivant :

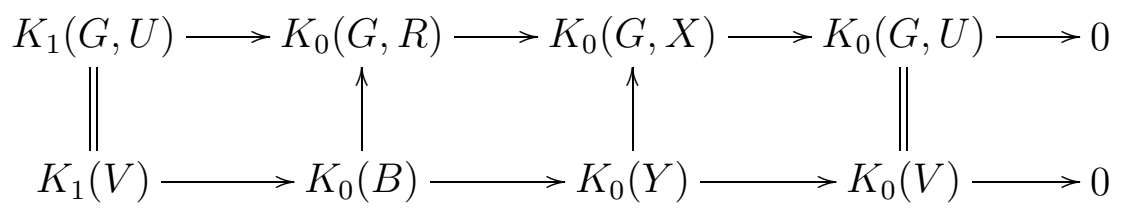

Dans ce diagramme, les lignes sont exactes, les colonnes sont données par pullback le long de $\pi$, et les isomorphismes verticaux viennent du fait que le revêtement $\pi_{\mid U}: U \rightarrow V$ est étale.

\subsection{Déterminant équivariant}

Donner une bonne définition de la première classe de Chern d'un G-faisceau localement libre, est un problème épineux. En effet le déterminant usuel det : $K(G, X) \rightarrow \operatorname{Pic}_{G} X$ est inadapté à la résolution de problème de type RiemannRoch, et le degré équivariant que nous avons introduit au chapitre 3 ne possède pas les propriétés attendues d'une classe de Chern (additivité, comportement par pullback ...).

L'introduction de cycles équivariants est en grande partie motivée par ce problème, et elle s'avère être une solution efficace. En effet le théorème 4.11 nous permet de définir ce déterminant de manière implicite. 


\section{Définition 4.13}

Soit $\mathcal{E}$ un $G$-faisceau sur $X$. On définit le déterminant équivariant de $\mathcal{E}$ comme étant le diviseur équivariant $\operatorname{det}_{G} \mathcal{E}$ dans $A^{1}(G, X)$ tel que $\left(\mathrm{rk} \mathcal{E}, \operatorname{det}_{G} \mathcal{E}\right)$ soit l'image de $[\mathcal{E}]$ par l'isomorphisme réciproque de l'isomorphisme du théorème 4.11.

\subsection{Théorème de Riemann-Roch équivariant}

\section{Théorème 4.14}

Soit $\mathcal{E}$ un $G$-faisceau cohérent sur $X$. On note $\chi(G, \mathcal{E})$ sa caractéristique d'Euler équivariante et $\operatorname{deg}_{G}(\mathcal{E})=\operatorname{deg}_{G}\left(\operatorname{det}_{G}([\mathcal{E}])\right)$. On a alors l'égalité dans $R_{k}(G)$ :

$$
\chi(G, \mathcal{E})=\operatorname{rk}(\mathcal{E}) \chi\left(G, \mathcal{O}_{X}\right)+\operatorname{deg}_{G}(\mathcal{E})
$$

\section{Démonstration :}

D'après le théorème 4.11 on a l'égalité suivante dans $K(G, X)$ :

$$
[\mathcal{E}]=\operatorname{rk} \mathcal{E}\left[\mathcal{O}_{X}\right]+\gamma\left(\operatorname{det}_{G} \mathcal{E}\right)
$$

Le théorème découle alors du lemme 4.3.

\section{Remarque :}

On peut déduire le théorème 3.11 (dans le cas modéré) du théorème 4.14. Pour cela, il suffit de montrer que pour tout faisceau inversible $\mathcal{L}$ on a :

$$
\operatorname{deg}_{e q} \mathcal{L}=\operatorname{deg}_{G} \mathcal{L}
$$

Cette égalité se montre facilement en représentant $\mathcal{L}$ par un diviseur $G$-invariant et en utilisant le lemme 4.4 . 


\section{Appendice A}

\section{Algèbre homologique dans les catégories de $G$-faisceaux}

Dans cet appendice, on rappele quelques théorèmes simples d'algèbre homologique, essentiellement tirés du Tohoku. Les preuves sont celles de Grothendieck, parfois quelque peu allégées par l'emploi de propriétés d'adjonction.

\section{Théorème A.1}

Soit $X$ un $G$-espace. La catégorie $\mathrm{Ab}(G, X)$ assez d'objets injectifs.

\section{Démonstration :}

Par souci de complétude, on repète ici la preuve de [13], Chapitre V. Cette preuve est basée sur le fait algébrique correspondant : pour tout anneau (éventuellement non-commutatif) $A$, la catégorie $\mathrm{Ab}(A)$ des $A$-modules a assez d'objets injectifs ([12], Théorème 1.2.2).

Soit à présent $\mathcal{F}$ un $G$-faisceau de groupes abéliens sur $X$. On va montrer que $\mathcal{F}$ peut être vu comme un sous objet d'un objet injectif dans la catégorie $\operatorname{Ab}(G, X)$. Pour cela fixons un point $P$ dans chaque orbite $O$ de $X$. D'après le fait rappelé ci-dessus, on peut trouver une injection $i_{P}: \mathcal{F}_{P} \hookrightarrow I_{P}$ du germe de $\mathcal{F}$ en $P$ dans un $\mathbb{Z}\left[G_{P}\right]$-module injectif. Pour les autres points de l'orbite de $P$ on pose $I_{g P}=I_{P}{ }^{g}$, autrement dit $I_{g P}$ est le $\mathbb{Z}\left[G_{g P}\right]$-module dont la structure de groupe abélien est isomorphe à celle de $I_{P}$ et sur lequel $G_{g P}$ agit grâce à la conjugaison $G_{g P}=g G_{P} g^{-1} . I_{g P}$ est un $\mathbb{Z}\left[G_{g P}\right]$-module injectif, et on dispose d'une injection $i_{g P}: \mathcal{F}_{g P} \hookrightarrow I_{g P}$ de $\mathbb{Z}\left[G_{g P}\right]$-modules uniquement déterminée par le fait que le diagramme de groupes abéliens :

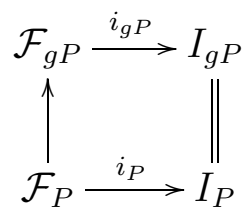

commute. Dans ce diagramme, la flèche verticale de gauche est celle induite par la $G$-linéarisation de $\mathcal{F}$. Posons $\mathcal{I}=\prod_{P} i_{P *} I_{P}$. On va montrer que $\mathcal{I}$ est un 
$G$-faisceau, qu'il est injectif comme objet de $\operatorname{Ab}(G, X)$, et qu'enfin $\mathcal{F}$ peut être vu comme un sous-faisceau de $\mathcal{I}$. Pour cela, on note $i_{O}: O \rightarrow X$ l'inclusion de l'orbite $O$ de $P$ dans $X$. L'existence de foncteurs :

$$
\mathrm{Ab}\left(G_{P}, P\right) \stackrel{\operatorname{Ind}_{G_{P}}^{G}}{\longrightarrow} \mathrm{Ab}(G, O) \stackrel{i_{O *}}{\longrightarrow} \mathrm{Ab}(G, X)
$$

montre que chaque $I_{P}$ définit un $G$-faisceau sur $X$, à savoir $i_{O *}\left(\operatorname{Ind}_{G_{P}}^{G} I_{P}\right)$. Comme $\mathcal{I}$ s'identifie comme faisceau sur $X$ au produit de ces faisceaux $\prod_{O} i_{O *}\left(\operatorname{Ind}_{G_{P}}^{G} I_{P}\right)$, on voit que $\mathcal{I}$ est muni naturellement d'une $G$-linéarisation. La fin de la démonstration est basée sur les propriétés d'adjonction des foncteurs que l'on vient de considérer. Le foncteur $\operatorname{Ind}_{G_{P}}^{G}$ admet comme quasi-inverse le foncteur de restriction $\left.{ }_{(\cdot \mid P}\right)$, quant au foncteur $i_{O *}$, il admet comme foncteur adjoint à gauche $i_{O}^{-1}$, ce qui montre que pour tout $G$-faisceau $\mathcal{G}$ :

$$
\operatorname{Hom}_{X}(\mathcal{G}, \mathcal{I})=\prod_{O} \operatorname{Hom}_{P}\left(i_{O}^{-1} \mathcal{G}_{\mid P}, I_{P}\right)
$$

Ceci permet d'une part de voir le foncteur $\operatorname{Hom}_{X}(\cdot, \mathcal{I})$ comme un foncteur composé de foncteurs exacts, ce dont on déduit que ce foncteur est exact, et donc que $\mathcal{I}$ est injectif dans $\operatorname{Ab}(G, X)$. D'autre part, cette égalité montre que les injections $\mathcal{F}_{P} \rightarrow I_{P}$ définissent un morphisme de $G$-faisceaux $\mathcal{F} \rightarrow \mathcal{I}$ qui est manifestement injectif.

Théorème A.2

Le foncteur d'oubli

$$
\begin{gathered}
\operatorname{Ab}(G, X) \longrightarrow \operatorname{Ab}(X) \\
\mathcal{F} \longrightarrow \widetilde{\mathcal{F}}
\end{gathered}
$$

envoie objets injectifs sur objets injectifs.

\section{Démonstration :}

Suivant Köck ([18]), on remarque tout d'abord qu'on peut associer à tout faisceau $\mathcal{G}$ sur $X$ le $G$-faisceau SymG défini par SymG $=\sum_{g \in G} g_{*} \mathcal{G}$, la $G$-linéarisation de SymG étant induite par la permutation des éléments de $G$. L'intérêt du foncteur $\operatorname{Sym}(\cdot)$ est qu'il est adjoint à gauche du foncteur d'oubli. Ceci étant dit, le reste de la démonstration est formel. Soit en effet $\mathcal{I}$ un $G$-faisceau injectif. Le foncteur $\operatorname{Hom}(\cdot, \widetilde{\mathcal{I}})$ est composé de deux foncteurs exacts, en effet

$$
\operatorname{Hom}(\cdot, \widetilde{\mathcal{I}})=\operatorname{Hom}(\cdot, \mathcal{I}) \circ \operatorname{Sym}(\cdot)
$$

$\widetilde{\mathcal{I}}$ est donc bien injectif.

\section{Théorème A.3}

Soit $f: X^{\prime} \rightarrow X$ un morphisme de $G$-espaces. Le foncteur image directe

$$
f_{*}: \mathrm{Ab}\left(G, X^{\prime}\right) \longrightarrow \operatorname{Ab}(G, X)
$$

envoie objets injectifs sur objets injectifs. 


\section{Démonstration :}

Analogue en tout point à la démonstration précédente, en utilisant le fait que le foncteur $f^{-1}$ est adjoint à gauche du foncteur $f_{*}$, et que $f^{-1}$ est exact.

On termine par un résultat technique bien connu ne concernant pas seulement les catégories de $G$-faisceaux, mais n'importe quelles catégories abéliennes :

\section{Théorème A.4}

Soient $\mathcal{A}, \mathcal{B}, \mathcal{C}$ trois catégories abéliennes, $\mathcal{A}$ et $\mathcal{B}$ ayant assez d'objects injectifs. Soient de plus $F: \mathcal{A} \rightarrow \mathcal{B}, G: \mathcal{B} \rightarrow \mathcal{C}$ deux foncteurs covariants exacts à gauche et $H=G \circ F$ le foncteur composé.

(i) Si $F$ est exact et envoie objects injectifs sur objets $G$-acycliques alors $R^{n} H=R^{n} G \circ F$ pour tout entier positif $n$.

(ii) Si $G$ est exact alors $R^{n} H=G \circ R^{n} F$ pour tout entier positif $n$.

\section{Démonstration :}

Soit $M$ un objet de $A$ et $M \rightarrow I^{\bullet}$ une résolution injective de $M$.

On se place dans le cas $(i)$. Alors $F M \rightarrow F\left(I^{\bullet}\right)$ est une résolution $G$-acyclique de $F M$, et donc $R^{n} G(F M)$ est l'homologie du complexe $G\left(F\left(I^{\bullet}\right)\right)$. Comme $G\left(F\left(I^{\bullet}\right)\right)=H\left(I^{\bullet}\right)$ on en déduit que ce groupe s'identifie à $R^{n} H M$.

Considérons à présent le cas (ii). Notons $C$ le complexe $F\left(I^{\bullet}\right)$. Il s'agit de comparer l'homologie de $C$ à celle du complexe $G(C)$. Mais comme $G$ est exact, l'homologie de $G(C)$ s'identifie à l'image par $G$ de l'homologie de $C$, d'où le résultat. 


\section{Bibliographie}

[1] Browder, William ; Katz, Nicholas M : Free actions of finite groups on varieties. II. Math. Ann. 260, 403-412 (1982).

[2] Baum, Paul ; Fulton, William; Quart, George : Lefschetz-Riemann-Roch for singular varieties. Acta Math. 143, 193-211 (1979).

[3] Benson, D. J : Representations and cohomology. I. Basic representation theory of finite groups and associative algebras. Cambridge Studies in Advanced Mathematics, 30 .

[4] Borel, Armand ; Serre, Jean-Pierre : Le théorème de Riemann-Roch. Bull. Soc. Math. France 861958 97-136.

[5] Chevalley, Claude; Weil, André : Über das Verhalten der Integrale 1. Gattung bei Automorphismen des Funktionenkoerpers. Abh. Math. Semin. Hamburg. Univ. 10, 358-361 (1934).

[6] Dress, Andreas W. M. : One more shortcut to Galois theory. Adv. Math. 110 (1995), no. 1, 129-140.

[7] Ellingsrud, Geir ; Lønsted, Knud : An equivariant Lefschetz formula for finite reductive groups. Math. Ann. 251, 253-261 (1980).

[8] Ellingsrud, Geir ; Lønsted, Knud : Equivariant $K$-theory for curves. Duke Math. J. 51 (1984), no. 1, 37-46.

[9] Edidin, Dan ; Graham, William : Equivariant intersection theory. Invent. Math. 131 (1998), no. 3, 595-634.

[10] Edidin, Dan ; Graham, William : Riemann-Roch for equivariant Chow groups. E-print math.AG/9905081.

[11] Green, J. A : On the indecomposable representations of a finite group. Math. Z. 70 1958/59 430-445.

[12] Godement, Roger : Topologie algébrique et théorie des faisceaux. Publications de l'Institut de Mathématique de l'Université de Strasbourg, XIII. Actualités Scientifiques et Industrielles, No. 1252. 
[13] Grothendieck, Alexandre : Sur quelques points d'algèbre homologique Tohoku Math. J., II. Ser. 9, 119-221 (1957).

[14] Hartshorne, Robin : Algebraic geometry. Graduate Texts in Mathematics, No. 52. Springer-Verlag, New York-Heidelberg, 1977.

[15] Fulton, William ; Lang, Serge : Riemann-Roch algebra. Grundlehren der Mathematischen Wissenschaften, 277. Springer-Verlag, 1985.

[16] Kani, Ernst : The Galois-module structure of the space of holomorphic differentials of a curve. J. reine angew. Math. 367 (1986), 187-206 .

[17] Köck, Bernhard : The Grothendieck-Riemann-Roch theorem for group schemes actions. Ann. Sci. Ecole Norm. Sup. (4) 31 (1998), no. 3, 415-458.

[18] Köck, Bernhard : Das Adams-Riemann-Roch-Theorem in der höheren äquivarianten K-Theorie. J. ReineAngew. Math. 421 (1991), 189-217.

[19] Lønsted, Knud : The singular points on the moduli spaces for smooth curves. Math. Ann. 266 (1984), no. 3, 397-402.

[20] Lønsted, Knud : On $G$-linebundles and $K_{G}(X)$. J. Math. Kyoto Univ. 23 (1983), no.4, 775-793.

[21] Manin, Ju. I. : Lectures on the $K$-functor in algebraic geometry. (Russian) Uspehi Mat. Nauk 241969 no. 5 (149) 3-86.

[22] Mézard, Ariane : Quelques problèmes de déformations en caractéristique mixte. Thèse, Institut Fourier, France (1998).

[23] Mumford, David : Abelian varieties. 2nd ed. Reprint. Studies in Mathematics, 5. Oxford University Press. XII, 279 p. (1985).

[24] Nakajima, Shoichi : On Galois module structure of the cohomology groups of an algebraic variety. Invent. Math. 75, 1-8 (1984).

[25] Nakajima, Shoichi : Galois module structure of cohomology groups for tamely ramified coverings of algebraic varieties. J. Number Theory 22, 115$123(1986)$.

[26] Nakajima, Shoichi : Action of an automorphism of order $p$ on cohomology groups of an algebraic curve. J. Pure Appl. Algebra 42, 85-94 (1986).

[27] Nielsen, H.Andreas : Diagonalizably linearized coherent sheaves. Bull. Soc. math. France 102, 85-97 (1974). 
[28] Quillen, Daniel : Higher algebraic $K$-theory. I. Algebraic $K$-theory, I: Higher $K$-theories (Proc. Conf., Battelle Memorial Inst., Seattle, Wash., 1972), pp. 85-147. Lecture Notes in Math., Vol. 341, Springer, Berlin 1973.

[29] Rzedowski-Calderón, Martha ; Villa-Salvador, Gabriel ; Madan, Manohar L. : Galois module structure of holomorphic differentials in characteristic $p$. Arch. Math. (Basel) 66 (1996), no. 2, 150-156.

[30] Serre, Jean-Pierre : Corps locaux. Actualités Scientifiques et Industrielles. 1296. Publications de l'Institut de Mathématique de l'Université de Nancago. VIII. Paris : Hermann. (1980).

[31] Serre, Jean-Pierre : Représentations linéaires des groupes finis. Hermann, Paris (1978).

[32] Thomason, R. W. : Une formule de Lefschetz en $K$-théorie équivariante algébrique. (A Lefschetz formula in equivariant algebraic $K$-theory). Duke Math. J. 68, No.3, 447-462 (1992).

[33] Thomason, R. W. : Algebraic K-theory of group scheme actions. Algebraic topology and algebraic $K$-theory (Princeton, N.J., 1983), 539-563, Ann. of Math. Stud., 113, Princeton Univ. Press, Princeton, NJ (1987).

[34] Burt Totaro : The Chow ring of a classifying space. E-print math.AG/9802097.

[35] Valentini, Robert C. ; Madan, Manohar L. : Automorphisms and holomorphic differentials in characteristic $p$. J. Number Theory 13, 106-115 (1981). 